\title{
METAL FUEL TUBE MANUFACTURE
}

S. R. Nemeth
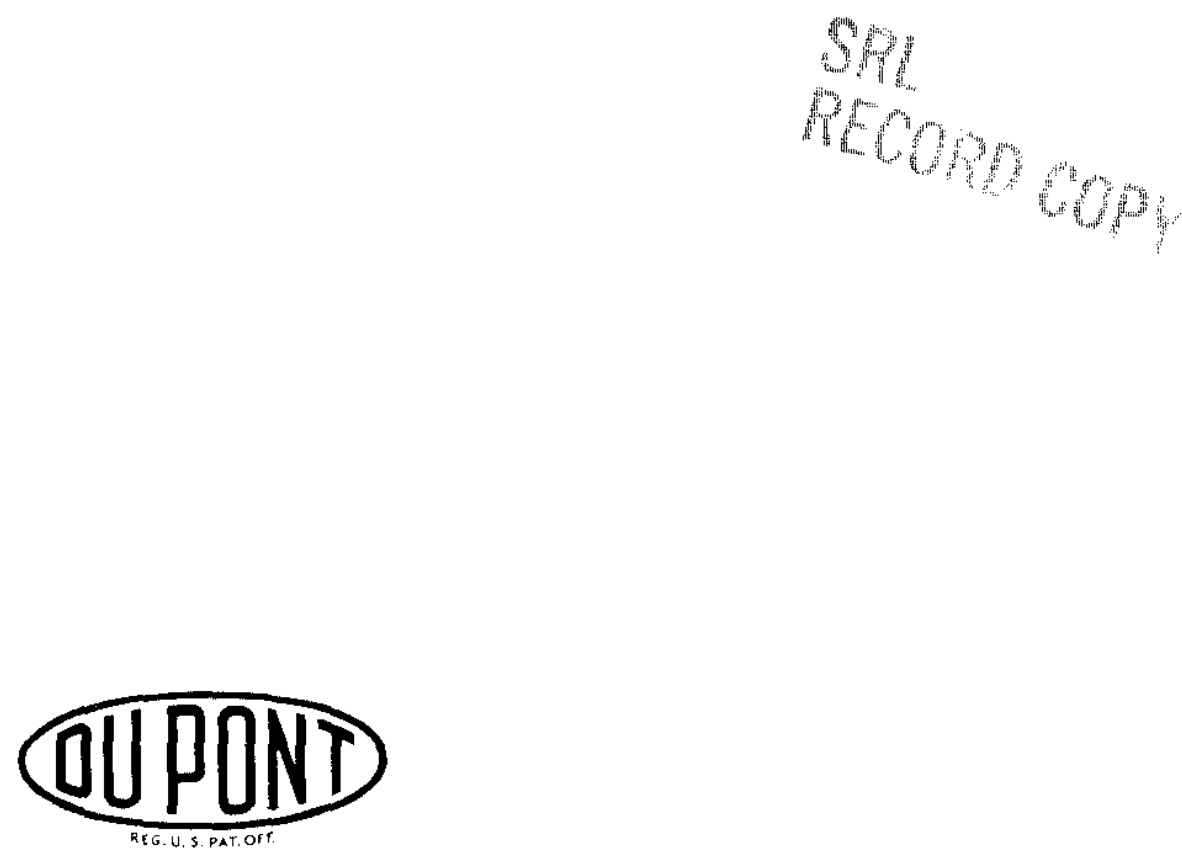

ISSUED BY

Savannah River Laboratory

Aiken, South Carolina 


\section{LEGAL NOTICE}

This report was prepared as an account of Government sponsored work. Neither the United States, nor the Commission, nor any person acting on behalf of the Commission:

A. Makes any warranty or representation, expressed or implied, with respect to the accuracy, completeness, or usefulness of the information contained in this report, or that the use of any information, apparatus, method, or process disclosed in this report may not infringe privately owned rights; or

B. Assumes any liabilities with respect to the use of, or for damages resulting from the use of any information, apparatus, method, or process disclosed in this report.

As used in the above, "person acting on behalf of the Commission" includes any employee or contractor of the Commission, or employee of such contractor, to the extent that such employee or contractor of the Commission, or employee of such contractor prepares, disseminates, or provides access to, any information pursuant to his employment or contract with the Commission, or his employment with such contractor.

Printed in USA. Price $\$ 3.00$

Available from the Clearinghouse for Federal Scientific and Technical Information, National Bureau of Standards,

U. S. Department of Commerce, Springfield, Virginia 
Metals, Ceramics, and Materials (TID-4500, 43rd Ed.)

METAL FUEL TUBE MANUSACTURE

\author{
Compiled by \\ Stephen R. Nemeth
}

Approved by

W. B. Delong, Director

Reactor Materials Section

July 1965

\author{
E. I. DU PONT DE NEMOURS \& COMPANY \\ SAVANNAH RIVER LABORATORY \\ AIKEN, SOUTH CAROLINA \\ CONTRACT AT(07.2).1 WITH THE \\ UNITED STATES ATOMIC ENERGY COMMISSION
}




\section{ABSTRACT}

Development and fabrication of tubular coextruded Zircaloy-clad uranium metal fuel elements for a heavy watermoderated-and-cooled power reactor is described. Approximately 200 experimental uranium tubular elements, 10 feet long, were fabricated of which 36 were irradiated. The remainder were used for evaluation of fabrication techniques and flow tests. Although the majority of tubes had cores of unalloyed uranium, urantum alloys with $2 \mathrm{w} / 0 \mathrm{Zr}$ and with Fe, S1, Al and Mo (approximately $0.2 \%$ total) were also included. Some 24 short-length (12-inch) elements were fabricated using brazed end seals rather than the integral end seals used for the long tubes. Fabrication techniques were developed for manufacture of $80 \mathrm{ZIrcaloy-clad} \mathrm{Zr}-235 \mathrm{U}$ alloy tubular driver elements, 10 feet long, for the FWCTR. Utilizing the techniques developed for uranium fuel tubes, a number of $T h-235 \mathrm{U}$ metal fuel tubes were coextruded to pllot the manufacture of $\mathrm{Th}-233 \mathrm{U}$ breeder reactor fuel. 


\section{CONTENTS}

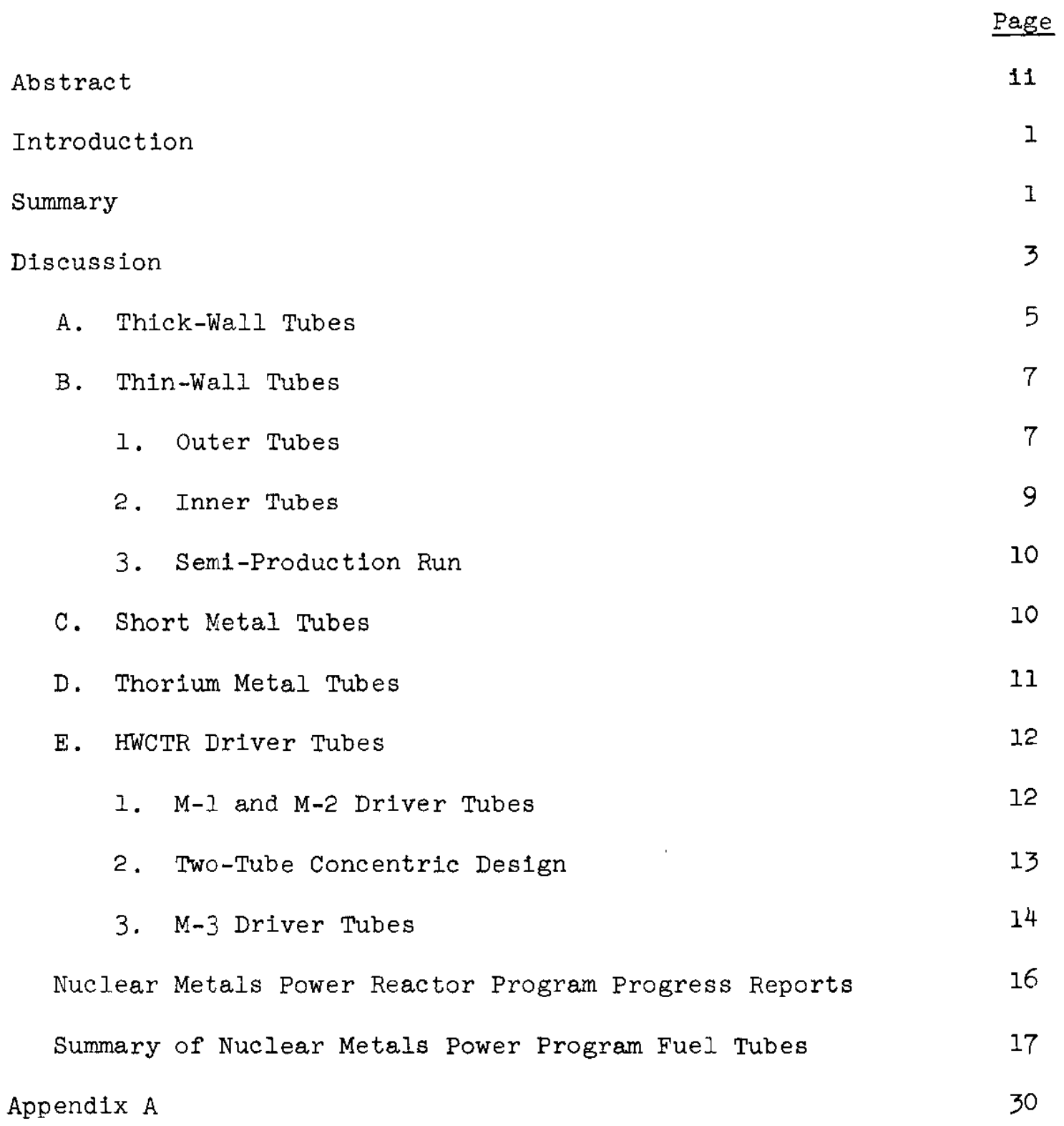




\title{
METAI FUEL TUBE MANUFACTURE
}

\begin{abstract}
INTRODUCTION
Metallic uranium fuel elements for heavy watermoderated-and-cooled power reactors have several advantages over oxide fuel. Among these are higher nuclear reactivity, higher thermal conductivity and potentially lower fabrication cost. A development effort for fabrication of tubular metallic fuel elements was successfuliy undertaken at Nuclear Metals, West Concord, Mass. Approximately 200 experimental uranium tubular elements were fabricated during the period 1957 to 1964. In addition, approximately 80 zirconium-235uranium alloy tubular fuel elements were fabricated for use as drivers for the HWCTR in which the metal test fuel was irradiated. This report summarizes the fabrication development effort on these various fuel tubes and reference documents that contain more detalled information.
\end{abstract}

\section{SUMMARY}

Coextruded Zlrcaloy-clad uranium-core tubular elements were investigated as potential fuels for use in heavy watermoderated-and-cooled power reactors. Nuclear Metals conducted the development work for fabrication of coextruded metal power fuel (1).

Zircaloy was used as the cladding material because of its low absorption cross section for neutrons, excellent corrosion resistance to water, and its high degree of metallurgical compatibility with uranium. This program included elements in which the core material was unalloyed uranium, uranium-2 w/o zirconium, and other uranium alloys with $\mathrm{Fe}, \mathrm{Si}, \mathrm{Al}$, and Mo.

(1) TID-7545, "Fuel Elements Conference, Paris,", November 1823, 1957, pages 157-181. 
In order to increase the power output per fuel assembly, the original thick-wall fuel tube (0.297" thick) was reduced to $0.180^{\prime \prime}$ and a two-tube concentric fuel element designed. The thinner wall not only allowed a greater power output but also reduced the fuel temperature and resulted in a lower level of cladding strain for the same percentage core volume change during irradiation. Following the fabrication of a series of experimental tubes, a set of fifteen inner and outer full-length fuel tubes was made for test 1rradiation. While the bulk of the fuel tubes were approximately 120 inches in length for use in the HWCTR, some tubes were 44 inches in length for use in the VBWR. The long tubes were all fabricated with integral end seals. A number of shortlength (about 12") experimental tubes were fabricated having brazed end seals. These short tubes provided means for irradiating a wider range of compositions and/or heat treatment in a minimum of reactor positions, as well as expediting postirradiation examination.

Fabrication techniques for manufacture of 10-foot long Zircaloy-clad zirconium-235uranium alloy tubular driver elements were developed for the HWCTR. Three full complements of tubes ( 24 per set) were fabricated and two sets of tubes were 1rradiated.

In connection with studies of heavy water-moderatedand-cooled Th- ${ }^{233} \mathrm{U}$ breeder reactors, a program was successfully undertaken to fabricate two pile-worthy Zircaloy-clad Th-1. $4 \mathrm{w} / \mathrm{o}$ $235 \mathrm{U}$ tubular elements which were later irradiated in the HWCTR. 
A tabulation of the power program fuel tubes is

presented at the end of this report; more complete detalis of each group of tubes are presented in Appendix A. Irradiation test data $w 111$ be reported in a supplement to this report.

\section{DISCUSSION}

During the initial phases of the Du Pont study of power reactors, a heavy water-moderated-and-cooled $100 \mathrm{MWe}$ (net electrical) reactor was contemplated. The Heavy Water Components Test Reactor (HWCTR) of $60 \mathrm{MW}(\mathrm{th})$ capacity was constructed for the purpose of testing the fuel alloys and assembly arrangements then visualized. The primary fuel element for the 100 MWe power reactor was consldered to be a Zircaloy-2-clad metal tubular design with a natural uranium-2 w/o zirconium core. The dimensions of this tube were $2.060^{\prime \prime}$ OD X $1.467^{\prime \prime}$ ID with a core length of about 10 feet for the components testing reactor and 15 feet for a full-scale reactor.

Under full-power operating conditions the maximum temperatures to which the tubular fuel elements were expected to be subjected were $295^{\circ} \mathrm{C}$ for cladding surface and $500^{\circ} \mathrm{C}$ for central core metal. Heat flux was expected to be approximately $400,000 \mathrm{pcu} / \mathrm{sq} . \mathrm{ft} / \mathrm{hr}$ with a maximum coolant temperature of $230^{\circ} \mathrm{C}$. Fuel elements were designed to be in a vertical position centered in ribbed Zircaloy-2 housing tubes with $0.400 "$ coolIng annul1. Initially, average exposure was estimated at 3000 MWD/tonne; 1 was raised later to a goal of $10,000 \mathrm{MWD} /$ tonne. 
The tubular metal fuel elements were fabricated by a coextrusion process with the fabrication development being done by Nuclear Metals. Preliminary production cost estimates on making 600 15-foot tubes per year (approximately 2 reactor charges) were $\$ 25 / 1 b$ of uranium with about half the cost being for the Zircaloy. The experimental tube cost was in the range of $\$ 80$ to $\$ 100 / 1 \mathrm{~b}$ of uranium, the high cost resulting primarily from inclusion of development costs.

The coextrusion process used for making the tubes consisted of the extrusion of a cylindrical billet, containing prefabricated core and cladding components, into a tubular shape. The extrusion was carried out at an elevated temperature $\left(600\right.$ to $\left.650^{\circ} \mathrm{C}\right)$. Conical extmusion dies were used so that both the core and cladding components could be made to flow in a streamlined fashion resulting in uniform core and cladding throughout the length of the tube. Particular care was taken in the shape of the billet components to prevent nonuniform cladding of the tube ends.

Individual billet components were made by conventional metal-working techniques. The core was made by elther extrusion or casting followed by machining to finished size. Cladding sleeves were machined from extruded Zircaloy stock and the end seals machined from Zircaloy forgings. The billet components were assembled and encased in copper which acted as a lubricant during the high-temperature extrusion. 
The coextruded tube was heat treated in 1ts copper extrusion jacket. After heat treatment, the copper was removed by ac1d dissolution from the Zircaloy cladding surface. Following inspection, the tube was etched and then autoclaved In high-temperature water $\left(395^{\circ} \mathrm{C}\right)$ and high pressure steam (1500 psi at $400^{\circ} \mathrm{C}$ ). Various in-process nondestructive tests were employed to insure core-to-clad bond quality and clad thickness.

The general types of tubes fabricated are described below. More complete deta1ls for individual experimental tubes are presented in the tabulated summary of power program fuel tubes and in Appendix $A$.

\section{A. Thick-Wall Tubes}

The original design for metal tubular power fuel elements was for a $2.060^{\prime \prime}$ OD $X 0.297^{\prime \prime}$ wall including $0.015^{\prime \prime}$ thick cladding. The tubes were approximately 10 feet long for the HWCTR, 4 feet for VBWR, and 15 feet for the contemplated 100 MWe power reactor. The tube wall thickness was based on an expected 500 to $550^{\circ} \mathrm{C}$ maximum central metal temperature, a heat flux of about $400,000 \mathrm{pcu} / \mathrm{ft}^{2}-\mathrm{hr}$, and a burmup to $3000 \mathrm{MWD} /$ tonne.

Uranium-2 w/o zirconium was the first choice for a core alloy because of its good extrusion compatibility with Zircaloy-2 cladding. This alloy was reported to exhibit a lower corrosion rate in high temperature water than unalloyed uranium, an advantage in the event of cladding fallure in the reactor. In addition, the irradiation 
stablilty was expected to be as good as or better than that of unalloyed uranium. A total of $42 \mathrm{U}-2 \mathrm{w} / 0 \mathrm{Zr}$ tubes were fabricated, 10 of which were the 4-foot tubes for VBWR. Seven full-length tubes and one short tube were irradiated at SRP, one full-length tube at NRU and one short tube at VBWR.

Postirradiation inspection revealed that splitting or cracking had occurred in some cores during irradiation. As a result, the core material in subsequent tubes was changed to unalloyed uranium, and studies were begun on other uranium alloys.

The Zircaloy cladding thickness was increased from $0.015^{\prime \prime}$ to $0.030^{\prime \prime}$ for the unalloyed uranium tubes so that the uranium content of the tubes would not be increased. Secondarliy, the th1cker cladding gave more adequate insurance of obtaining satisfactory tubes despite the greater difference in fabrication properties of unalloyed uranium and the Zircaloy-2 cladding and end seals.

Thirteen thick-wall tubes with unalloyed uranium cores, one with U-1 w/O Si core and one with U-1.5 w/O Mo core were fabricated before the fuel design was changed to include thinner wall thickness. Reduction in wall thickness would reduce the maximum central core temperatures and, hopefully, increase exposures to higher levels than those originally specifled. Two unalloyed uranium tubes were irradiated prior to adoption of the change. 
Fabrication details for the thick-wall tubes are presented in Appendix A, Sections 1-6, 8 and 10-13.

B. Thin-Wall Tubes

In early 1960 the single-tube, thick-wall fuel design was changed to a concentric two-tube design having thin walls. The following table compares the dimensions of the concentric tubes with the single thick-wall tube.

\begin{tabular}{|c|c|c|c|}
\hline \multirow{2}{*}{ Item } & \multirow{2}{*}{$\begin{array}{l}\text { Single } \\
\text { Tube } \\
\text { (Inches) }\end{array}$} & \multicolumn{2}{|c|}{ Concentric Tubes } \\
\hline & & $\frac{\text { Outer }}{\text { (Inches) }}$ & $\frac{\text { Inner }}{\text { (1nches) }}$ \\
\hline $\begin{array}{l}\text { Outside diameter } \\
\text { Inside diameter } \\
\text { Cladding thlckness } \\
\text { Core thickness } \\
\text { Total wall thickness }\end{array}$ & $\begin{array}{l}2.060 \\
1.462 \\
0.030 \\
0.237 \\
0.299\end{array}$ & $\begin{array}{l}2.060 \\
1.700 \\
0.025 \\
0.130 \\
0.180\end{array}$ & $\begin{array}{l}1.020 \\
0.660 \\
0.025 \\
0.130 \\
0.180\end{array}$ \\
\hline
\end{tabular}

Development and fabrication of the outer and inner thin-wall fuel elements was performed concurrently.

1. Outer Tubes

Of the 55 experimental thin-wall outer tubes fabricated, 44 had unalloyed uranium cores. Five of the 55 tubes had enriched uranium cores; two unalloyed full-length tubes were enriched to $2.1 \% 235 \mathrm{U}$ for Irradiation in HWCTR; and three alloyed short tubes were enriched to $3.0 \% 235 \mathrm{U}$ for Irradiation in VBWR. The tubes with uranium alloy cores included four with $\mathrm{U}-1 \mathrm{w} / \mathrm{O} \mathrm{SI}$ (one with $\mathrm{U}$ enriched to $3 \% 235 \mathrm{U}$ ); $81 \times$ with $\mathrm{U}-1.5 \mathrm{w} / \mathrm{O}$ Mo (two with $\mathrm{U}$ enriched to $3 \% 235 \mathrm{U}$ ); and one with U-0.3 w/O Al-0.5 w/o Si. 
Four of the unalloyed uranium tubes were fabricated with different clad thicknesses to evaluate (1) the effect of cladding restraint on fuel swelling durIng irradiation and (2) whether the direction of swelling could be controlled. Dimensions of these tubes are listed below.

"Restraint" Tubes

\begin{tabular}{|c|c|c|c|c|}
\hline & $\begin{array}{l}\text { Normal Tube } \\
\text { (Inches) }\end{array}$ & $\begin{array}{l}\text { (1ype A } \\
\text { (inches) }\end{array}$ & $\begin{array}{l}\text { (1ype } B \\
\text { (inches) }\end{array}$ & $\begin{array}{l}\text { Iype c } \\
\text { (Inches) }\end{array}$ \\
\hline $\begin{array}{l}\text { Outside diameter } \\
\text { Inside diameter }\end{array}$ & $\begin{array}{l}2.060 \\
1.700\end{array}$ & $\begin{array}{l}2.060 \\
1.560\end{array}$ & $\begin{array}{l}2.060 \\
1.670\end{array}$ & $\begin{array}{l}2.060 \\
1.670\end{array}$ \\
\hline $\begin{array}{c}\text { Cladding thickness, } \\
\text { outside }\end{array}$ & 0.025 & 0.060 & 0.025 & 0.040 \\
\hline $\begin{array}{c}\text { Cladding thickness, } \\
\text { inside }\end{array}$ & 0.025 & 0.060 & 0.040 & 0.025 \\
\hline Core thickness & 0.130 & 0.130 & 0.130 & 0.130 \\
\hline Total wall thickness & 0.180 & 0.250 & 0.195 & 0.195 \\
\hline
\end{tabular}

Among the developments accomplished with the experimental tubes were the establishment of basic information for fabrication of the thin-wall tubes, such as methods for grain refinement of the billet core prior to machining and billet preshape design which resulted in obtaining more uniform cladding of the coextruded tubes. The process developed for grain refinement of the billet core consisted of beta treatment of the core stock followed by a single extrusion at a low reduction ratio. This replaced the one initially used which consisted of repeated alpha extrusions with intermediate upsets and which resulted in low metal ylelds due to the large amount of machining involved. A b1llet end shape ("preshape") which incorporated several different tapers was found to result In more uniform cladding thickness in the taper region between the core and end cap of the extruded tubes than did spherical preshapes. 
Six of the outer tubes were irradiated individually. These included two unalloyed natural uranium tubes, two unalloyed uranium with $2.1 \% 235 \mathrm{v}$ enrichment, one Type A restraint tube, and one short $U-1.5 \mathrm{w} / 0$ Mo tube enriched to $3.0 \% 235 \mathrm{~J}$ (assembly polsoned to some degree; tube originally scheduled for VBWR).

Fabrication details for the thin-wall outer tubes are presented in Appendix A, Sections 14-16, 18-19, 21-22, 24-25 and 27-28.

2. Inner Tubes

Twenty-one experimental thin-wall inner tubes were fabricated, all with unalloyed uranium cores. Five were enriched to $2.1 \% 2350$. Three were fabricated with different claddin thicknesses to evaluate the effect of cladding restraint, as was done for the outer tubes. Dimensions of these tubes are listed below.

\begin{tabular}{|c|c|c|c|}
\hline & $\begin{array}{l}\text { Norma 1 Tube } \\
\text { (Inches) }\end{array}$ & $\begin{array}{l}\text { Restrail } \\
\text { Type D } \\
\text { (Inches) }\end{array}$ & $\begin{array}{l}\text { Tubes } \\
\text { Type E } \\
\text { (1nches) }\end{array}$ \\
\hline Outs1de diameter & 1.020 & 1.020 & 1.020 \\
\hline Inside diameter & 0.660 & 0.520 & 0.630 \\
\hline $\begin{array}{c}\text { Cladding thickness, } \\
\text { outside }\end{array}$ & 0.025 & 0.060 & 0.040 \\
\hline $\begin{array}{c}\text { Cladding thickness, } \\
\text { inside }\end{array}$ & 0.025 & 0.060 & 0.025 \\
\hline Core thickness & 0.130 & 0.130 & 0.130 \\
\hline Total wall thickness & 0.180 & 0.250 & 0.195 \\
\hline
\end{tabular}

Four of the unalloyed, natural uranium, inner, thinwall tubes were irradiated individually. The process for fabricating the inner tubes was similar to that for the outer tubes. 
Deta11s are presented in Appendix A, Sections 17, 20, 23 and 26.

3. Sem1-Production Run

Fifteen sets of unalloyed, natural uranium, thinwall tubes were successfully fabricated for irradiation as nested pairs in HWCTR. These tubes were made using the fabrication techniques developed for making the individual outer and inner experimental thin-wall tubes. Seven fuel assemblies using seven each of these tubes were Irradiated in HWCTR with Zircaloy-2 spiral ribbontype spacers between the two fuel tubes. Irradiation testing of these tubes was terminated following early fallure of two assemblies as a result of rapid fretting corrosion of the zircaloy cladding where the spacers had vibrated against the tube walls.

Fabrication details for these tubes are presented In Appendix A, Sections 34 and 35.

\section{Short Metal Tubes}

A short length (11t") tubular fuel element with brazed end seals was designed to provide a means for 1rradiating a wide range of compositions and/or heat treatments in a minimum number of reactor positions. Postirradiation examination was expedited by the use of short tubes because they could be introduced into high level caves directly and without the cutting and handling required for long elements. The core wall thickness of these tubes was approximately $40 \%$ greater than the previous thin-wall design to obtain a higher core temperature during irradiation. Twenty-four short 
elements, consisting of four variations of core heat

treatment and composition, were fabricated from the long

extruded tubes.

Tube

Numbers

Composition

$\begin{array}{ll}150,151 & \text { Unalloyed uranium } \\ 152,153, & \text { U-350 ppm Fe-900 ppm A1 } \\ 154 & \\ 155 & \text { U-350 ppm Fe-900 ppm A1-500 ppm Si-1000 ppm Mo } \\ 157,158 & \text { U-350 ppm Fe-900 ppm A1-300 ppm Si }\end{array}$

Tube

Numbers

Billet Core Heat Treatment Prior to Extrusion Cladding

150,151 Triple beta-treated followed by series of extrusions and intermediate upsets.

152,153, Beta-treated - a1r-cooled followed by beta-treated -

155,157, o11 quench.

154 Gamma-treated - furnace-cooled.

Two reactor assemblies containing a total of five

short tubes from each of Nos. 153, 154 and 158 for a total

of fifteen short tubes were irradiated in the HWCTR. Fabri-

cation details for these tubes are presented in Appendix A,

Section 29.

D. Thorium Metal Tubes

The Du Pont program on power reactor development included the assessment of the heavy water-moderated-andcooled breeder reactor concept operating on the Th-233U fuel cycle. Fabrication of several $\mathrm{Th}-235 \mathrm{U}$ alloy fuel elements was undertaken as a step in the evaluation of a prototype fuel element. The coextruded test elements, 2.540 " oD X 
$1.830^{\prime \prime}$ ID, $0.290^{\prime \prime}$ thick core and $0.030^{\prime \prime}$ cladding thickness, were designed for a serles of Irradiations in HWCTR. Four thorium-natural uranium and three thorium-enriched uranium elements were made. Two of the enriched elements were irradiated in the HWCTR. Fabrication details for these tubes are presented in Appendix A, Section 33.

E. HWCTR Driver Tubes

1. M-1 and $M-2$ Driver Tubes

Eight prototype tubular driver elements were made with natural uranium to develop the process for fabricating enriched tubes for HWCTR. After successful development, three enriched tubes were fabricated, two of which were for test 1rradiation at SRP. To produce 50 acceptable tubes for HWCTR, a production run of 56 tubes was made.

All tubes had $\mathrm{z} 1$ rconium- $9.3 \mathrm{w} / \mathrm{o}$ fully enriched uranium cores with Zircaloy-2 end seals. Twenty-seven tubes were clad with Zircaloy-2, twenty-seven with Zircaloy-4, (1) and two with Zircaloy-2 on the outer surface and Zircaloy-4 on the inner surface. The change to Zircaloy-4 was made midway during fabrication in view of an expected lesser amount of hydrogen absorbed during irradiation by Zircaloy -4 as compared to Zircaloy-2. Nominal dimensions of the tubes were $2.300^{\prime \prime}$ OD X $0.167^{\prime \prime}$ wall including $0.015^{\prime \prime}$ cladding thickness. of the 56 production elements only 3 were not consldered of irradiation quality because of minor cladding defects.

(1) Current designation of this alloy is low nickel-Zircaloy-2. 
Forty-eight of the tubes were 1rradiated in HWCTR, 24 as the M-1 driver set and 24 as the M-2 driver set, with satisfactory results. The remaining 8 enriched tubes, including the 3 with minor cladding defects, were placed in storage at SRP.

Fabrication details for these tubes are presented In Appendix A, Sections 7, 9 and 36.

2. Two-Tube Concentric Design

To permit operation of the HWCTR at higher flux levels in test fuel positions, a concentric two-tube driver fuel design was developed. The two-tube rested design provided greater heat transfer area than was avallable in the one-tube case. The uranium loading per driver assembly was also increased from 108 grams/ft to $120 \mathrm{grams} / \mathrm{ft}$. Four outer and five inner prototype tubes were fabricated. Comparison of the dimensions of these tubes to the single-tube design follows:

\begin{tabular}{|c|c|c|c|}
\hline \multirow{3}{*}{$\begin{array}{l}\text { Outside diameter, inches } \\
\text { Inside dlameter, inches } \\
\text { Cladding thickness, m1ls } \\
\text { Core thickness, mils } \\
\text { Total wall thickness, m1ls }\end{array}$} & $\begin{array}{c}\text { Single } \\
\text { Tube } \\
\end{array}$ & \multicolumn{2}{|c|}{$\frac{\text { Concentric Tubes }}{\text { outer Inner* }}$} \\
\hline & $\begin{array}{l}2.300 \\
1.966\end{array}$ & $\begin{array}{l}2.700 \\
2.430\end{array}$ & $\begin{array}{l}1.720 \\
1.400\end{array}$ \\
\hline & $\begin{array}{r}15 \\
137 \\
167\end{array}$ & $\begin{array}{r}15 \\
105 \\
135\end{array}$ & $\begin{array}{l}15 \\
130 \\
160\end{array}$ \\
\hline \multicolumn{4}{|c|}{ 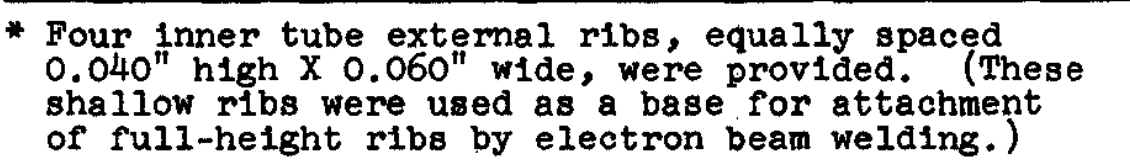 } \\
\hline
\end{tabular}

The core alloy was changed from zirconium-9.3 w/o enriched uranium to Zircaloy-2 $6.5 \mathrm{w} / \mathrm{o}$ enriched uranium. 
The lower uranium concentration was used to utilize core thicknesses in the range in which prior experience was ava1lable; the new development costs incident to the use of thinner walls of $9.3 \mathrm{w} / 0$ uranium were thus avolded.

The inner tube, originally concelved with a smooth outer surface, was modified to incorporate shallow Zircaloy ribs on this surface to provide a suitable base for subsequent electron beam welding of full-height Zircaloy ribs for spacing the concentric tubes. Electron beam welding of the full-helght ribs to the extruded shallow ribs was satisfactorily demonstrated on a 70-1nch length of a prototype inner tube. Development was terminated on the two-tube driver design because the higher cost of producing elements of this type did not appear to be justifled by their difference in performance over that of single-tube arivers.

Fabrication details of the two-tube design are presented in Appendix A, Sections 30 and 31.

\section{3. $\mathrm{M}-3$ Driver Tubes}

To maintain the reactivity characteristics of the two-tube design, the entire uranium loading of the inner tube was added to that of the outer tube. To attain the desired higher flux in the test positions, It was necessary to reduce the coolant water temperature 
to permit a higher burnout safety factor and hence higher heat transfer rate of the driver tubes. Prior to fabricating the 28 Zircaloy-2 $11.1 \mathrm{w} / 0$ fully enriched uranium driver tubes, 3 prototypes were made using natural uranium. The design of this element is extermally identical to the outer tube of the two-tube driver element described above. The uranium in the core alloy was increased, placing in this single tube all the uranium that was originally distributed between the two tubes of the earlier design.

Four of the 28 production tubes were not considered irradiation candidates because of minor defects. Operation of the HWCTR was terminated before any of this set of driver tubes could be irradiated. Fabrication deta1ls are presented in Appendix A, Sections 32 and 37. 


\section{NUCLEAR METALS}

Power Reactor Program Progress Reports

\begin{tabular}{|c|c|c|c|c|c|}
\hline $\begin{array}{r}\text { NMI- } 4350 \\
4351 \\
4353 \\
4354 \\
4355\end{array}$ & $\begin{array}{l}5 / 30 / 57 \\
6 / 24 / 57 \\
7 / 20 / 57 \\
8 / 21 / 57 \\
9 / 21 / 57\end{array}$ & $\begin{array}{l}-6 / 23 / 57 \\
-7 / 19 / 57 \\
-8 / 20 / 57 \\
-9 / 20 / 57 \\
-10 / 16 / 57\end{array}$ & $\begin{array}{r}\text { NMI-7228 } \\
7229 \\
7230 \\
7231 \\
7232\end{array}$ & $\begin{array}{l}5 / 1 / 60 \\
6 / 1 / 60 \\
7 / 1 / 60 \\
8 / 1 / 60 \\
9 / 1 / 60\end{array}$ & $\begin{array}{l}5 / 31 / 60 \\
-6 / 30 / 60 \\
-7 / 31 / 60 \\
-8 / 31 / 60 \\
-9 / 30 / 60\end{array}$ \\
\hline $\begin{array}{r}\text { NMI- } 4356 \\
4357 \\
4358 \\
4359 \\
4361\end{array}$ & $\begin{array}{r}10 / 17 / 57 \\
11 / 18 / 57 \\
1 / 26 / 58 \\
2 / 21 / 58 \\
3 / 19 / 58\end{array}$ & $\begin{array}{lr}- & 11 / 17 / 57 \\
- & 1 / 25 / 58 \\
- & 2 / 20 / 58 \\
- & 3 / 18 / 58 \\
- & 4 / 11 / 58\end{array}$ & $\begin{array}{r}\text { NMI-7233 } \\
7234 \\
7235 \\
7236 \\
7237\end{array}$ & $\begin{array}{c}10 / 1 / 60 \\
11 / 1 / 60 \\
12 / 1 / 60 \\
1 / 1 / 61 \\
2 / 1 / 61\end{array}$ & $\begin{array}{l}-10 / 31 / 60 \\
-11 / 30 / 60 \\
=12 / 31 / 60 \\
=\quad 1 / 31 / 61 \\
-\quad 2 / 28 / 61\end{array}$ \\
\hline $\begin{array}{r}\text { NMI- } 4362 \\
4363 \\
4366 \\
4368 \\
4369\end{array}$ & $\begin{array}{l}4 / 12 / 58 \\
5 / 7 / 58 \\
6 / 7 / 58 \\
7 / 6 / 58 \\
8 / 1 / 58\end{array}$ & $\begin{array}{l}-5 / 6 / 58 \\
=\quad 6 / 6 / 58 \\
-\quad 7 / 6 / 58 \\
-7 / 31 / 58 \\
-8 / 31 / 58\end{array}$ & $\begin{array}{r}\text { NMI-7238 } \\
7239 \\
7240 \\
7241 \\
7242\end{array}$ & $\begin{array}{l}3 / 1 / 61 \\
4 / 1 / 61 \\
5 / 1 / 61 \\
6 / 1 / 61 \\
8 / 1 / 61\end{array}$ & $\begin{array}{ll} & 3 / 31 / 61 \\
- & 4 / 30 / 61 \\
- & 5 / 31 / 61 \\
- & 7 / 31 / 61 \\
- & 8 / 31 / 61\end{array}$ \\
\hline $\begin{array}{r}\text { NMI- } 4381 \\
4382 \\
4383 \\
4384 \\
4385\end{array}$ & $\begin{array}{r}9 / 1 / 58 \\
10 / 1 / 58 \\
11 / 1 / 58 \\
12 / 1 / 58 \\
1 / 1 / 59\end{array}$ & $\begin{array}{l}-9 / 30 / 58 \\
-10 / 31 / 58 \\
-11 / 30 / 58 \\
-12 / 31 / 58 \\
-11 / 31 / 59\end{array}$ & $\begin{array}{r} \\
\text { NMI-7243 } \\
7244 \\
7245 \\
7246 \\
7247\end{array}$ & $\begin{array}{l}9 / 1 / 61 \\
10 / 1 / 61 \\
11 / 1 / 61 \\
12 / 1 / 61 \\
1 / 1 / 62\end{array}$ & $\begin{array}{rr} & 9 / 30 / 61 \\
- & 10 / 31 / 61 \\
-11 / 30 / 61 \\
-12 / 31 / 61 \\
-\quad 1 / 31 / 62\end{array}$ \\
\hline $\begin{array}{r}\text { NMI- } 4386 \\
4392 \\
4393 \\
4394 \\
4395\end{array}$ & $\begin{array}{l}2 / 1 / 59 \\
3 / 1 / 59 \\
4 / 1 / 59 \\
5 / 1 / 59 \\
6 / 1 / 59\end{array}$ & $\begin{array}{l}-2 / 28 / 59 \\
-3 / 31 / 59 \\
-4 / 30 / 59 \\
-5 / 31 / 59 \\
-6 / 30 / 59\end{array}$ & $\begin{array}{r}\text { NMI-7248 } \\
7249 \\
7250 \\
7251 \\
7252\end{array}$ & $\begin{array}{l}2 / 1 / 62 \\
3 / 1 / 62 \\
4 / 1 / 62 \\
5 / 1 / 62 \\
6 / 1 / 62\end{array}$ & $\begin{array}{l}-2 / 28 / 62 \\
-\quad 3 / 31 / 62 \\
-\quad 4 / 30 / 62 \\
-\quad 5 / 31 / 62 \\
-\quad 6 / 30 / 62\end{array}$ \\
\hline $\begin{array}{r}\text { NMI- } 4396 \\
4397 \\
4398 \\
7221 \\
7222\end{array}$ & $\begin{array}{r}7 / 1 / 59 \\
8 / 1 / 59 \\
9 / 1 / 59 \\
10 / 1 / 59 \\
11 / 1 / 59\end{array}$ & $\begin{array}{l}-7 / 31 / 59 \\
-8 / 31 / 59 \\
-9 / 30 / 59 \\
-10 / 31 / 59 \\
-11 / 30 / 59\end{array}$ & $\begin{array}{r}\text { NMI-7253 } \\
7254 \\
7255 \\
7256 \\
7257\end{array}$ & $\begin{array}{r}7 / 1 / 62 \\
8 / 1 / 62 \\
9 / 1 / 62 \\
10 / 1 / 62 \\
11 / 1 / 62\end{array}$ & $\begin{array}{l}-\quad 7 / 31 / 62 \\
-\quad 8 / 31 / 62 \\
-\quad 9 / 30 / 62 \\
-10 / 31 / 62 \\
-11 / 30 / 62\end{array}$ \\
\hline $\begin{array}{r}\text { NMI-7223 } \\
7224 \\
7225 \\
7226 \\
7227\end{array}$ & $\begin{array}{r}12 / 1 / 59 \\
1 / 1 / 60 \\
2 / 1 / 60 \\
3 / 1 / 60 \\
4 / 1 / 60\end{array}$ & $\begin{array}{l}-12 / 31 / 59 \\
-1 / 31 / 60 \\
-2 / 29 / 60 \\
-3 / 31 / 60 \\
-4 / 30 / 60\end{array}$ & $\begin{array}{r}\text { NMI-7258 } \\
7259 \\
7260\end{array}$ & $\begin{array}{r}12 / 1 / 62 \\
1 / 1 / 63 \\
2 / 1 / 63\end{array}$ & $\begin{array}{l}-12 / 31 / 62 \\
-\quad 1 / 31 / 63 \\
-\quad 4 / 30 / 63\end{array}$ \\
\hline
\end{tabular}




\section{SUMMARY OF NUCLEAR METALS POWER PROGRAM FUEL TUBES}

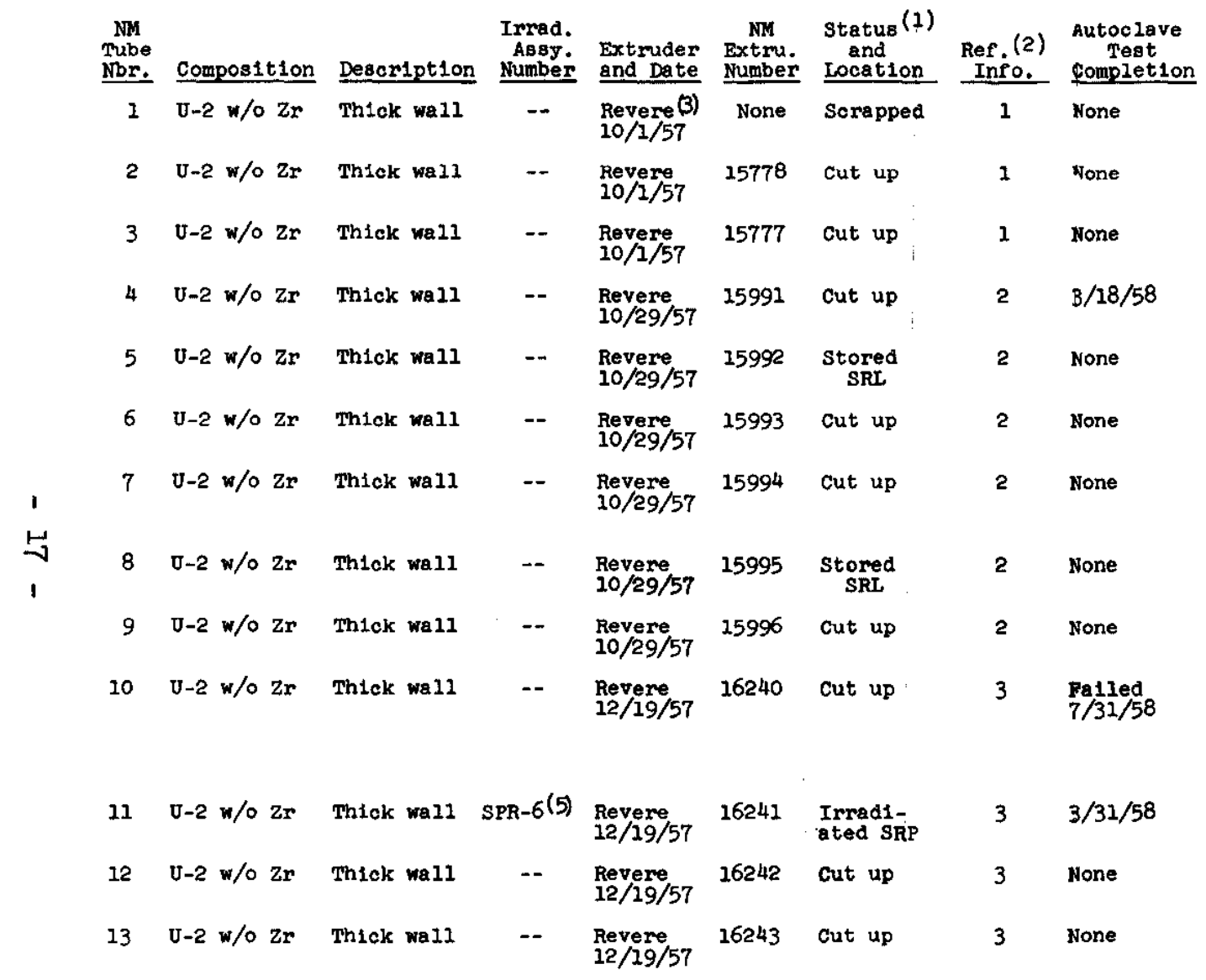

\section{Not extmided. Copper can torn off because not cen-
tered in liner. Disassembled.}

Tandem extrusion of 3 cores. Interface design varied.

Changes in interface design. Destructiveiy examined.

Used for etching experiments. Samples used for $800^{\circ} \mathrm{C}$ heat treatment studies $(4)$

Used for SRL flow atudies and HWCTR zero power tests. .

F1rst double evacuated and sealed billet. Destructively examined.

First full scale $880^{\circ} \mathrm{C}$ heat treatment. Two feet used for burst tests; one foot used for X-ray orlentation

Used for NRU flow tests at SRL and HWCTR zero power tests. Not sultable for irradiation.

Tandem extrusion of 3 cores. All 3 sections used for vertical heat treatment and corrosion studies.

Zr plcked up on die during extmusion. Rear cladding thin. Heat treated on graphite cylinder. Falled durIng autoclave test. Repalred and heat treated on Mo pads with no restralnt. Used for metallurgical structure studies.

Heat treated at $800^{\circ} \mathrm{C}$

Heat treated on tantalum pad. Used for corrosion tudies.

Rear cladding thin. Nail hole over core $5 \mathrm{mils}$ deep. Heat treated on stalnless steel pad. Used for corro
(1) Tube status as of $3 / 1 / 65$.

(2) Reference Information is presented in Appendix A, listed under appropriate section number.

(3) Revere Copper \& Brass Co., Detrolt, Mich1gan.
(4) Unless otherwise speclfied, heat treatment of $\mathrm{U}-2 \mathrm{w} / \mathrm{O} \mathrm{Zr}$ tubes was
$880^{\circ} \mathrm{C}$ for 8 hours followed by cooling at a rate equivalent to air cooting.

(5) SPR - Savannah Production Reactor Test Fuel number. 
Summary of Nuclear Metals Power Program Fuel Tubes (Continued)

\begin{tabular}{|c|c|c|c|c|c|c|c|c|c|}
\hline $\begin{array}{l}\text { NMI } \\
\text { Tube } \\
\text { Nor. }\end{array}$ & Composition & Description & $\begin{array}{l}\text { Irrad. } \\
\text { Assy. } \\
\text { Number }\end{array}$ & $\begin{array}{l}\text { Extruder } \\
\text { and Date }\end{array}$ & $\begin{array}{l}\begin{array}{c}\text { NM } \\
\text { Extru. } \\
\text { Number }\end{array} \\
\end{array}$ & $\begin{array}{l}\text { Status } \\
\text { and } \\
\text { Lodation } \\
\end{array}$ & $\begin{array}{l}\text { Ref. } \\
\text { Info, }\end{array}$ & $\begin{array}{l}\text { Autoclave } \\
\text { Test } \\
\text { Completion }\end{array}$ & Comments \\
\hline 14 & $\mathrm{U}-2 \mathrm{w} / 0 \mathrm{Zr}$ & Thick wall & - & $\begin{array}{l}\text { Revere } \\
12 / 19 / 57\end{array}$ & 16244 & $\begin{array}{l}\text { Stored } \\
\text { SRL }\end{array}$ & 3 & $3 / 26 / 58$ & $\begin{array}{l}\text { Tantalum pits on surface. Used for HWCTR zero power } \\
\text { tests. May be suitable for Irradiation; however, warp- } \\
\text { ing did occur during autoclaving and may be expected } \\
\text { to warp if Irradiated. }\end{array}$ \\
\hline 15 & $\mathrm{U}-2 \mathrm{w} / \mathrm{Or}$ & Thick wall & SPR-2 & $\begin{array}{l}\text { Revere } \\
1 / 30 / 58\end{array}$ & 16431 & $\begin{array}{l}\text { Irradia- } \\
\text { ted isRP }\end{array}$ & 3 & $B / 28 / 58$ & None. \\
\hline 16 & $\mathrm{U}-2 \mathrm{w} / 0 \mathrm{Zr}$ & Th1ck wall & -- & $\begin{array}{l}\text { Revere } \\
1 / 30 / 58\end{array}$ & 16432 & $\begin{array}{l}\text { Stored } \\
\text { SRL }\end{array}$ & 3 & $10 / 28 / 58$ & $\begin{array}{l}\text { Heat treated on stainless steel. Used for HWCTR zero } \\
\text { power tests. }\end{array}$ \\
\hline 17 & $\mathrm{U}-2 \mathrm{w} / 0 \mathrm{Zr}$ & Thick wall & -- & $\begin{array}{l}\text { Revere } \\
3 / 11 / 58\end{array}$ & 16817 & Cut up & 4 & None & $\begin{array}{l}\text { Mandrel broke during extmusion. Used in as-extruded } \\
\text { condition for corrosion tests. }\end{array}$ \\
\hline 18 & $\mathrm{U}-2 \mathrm{~W} / \mathrm{O} \mathrm{Zr}$ & Th1ck w811 & -- & $\begin{array}{l}\text { Revere } \\
3 / 11 / 58\end{array}$ & 16818 & $\begin{array}{c}\text { Stored } \\
\text { SRI } \\
\vdots\end{array}$ & 4 & -- & $\begin{array}{l}\text { Thin cladding at rear end seal. Heat treated on both } \\
\text { graphite and Mo pads. one end had white corrosion pro- } \\
\text { duct. End seal section removed for U contamination } \\
\text { analysis. Surface has several scratches. Used for } \\
\text { HWCTR zero power tests. }\end{array}$ \\
\hline 19 & $\mathrm{U}-2 \mathrm{w} / \mathrm{o} \mathrm{z}_{\mathbf{r}}$ & Thick wall & $\mathrm{SPR}-3$ & $\begin{array}{l}\text { Revere } \\
3 / 21 / 58\end{array}$ & 16819 & Impad1a- & 4 & $6 / 30 / 58$ & Heat treated on stalnless steel. \\
\hline 20 & $\mathrm{U}-2 \approx / \circ \mathrm{Zr}$ & Th1ck wall & -- & $\begin{array}{l}\text { Revere } \\
3 / 11 / 58\end{array}$ & 16820 & $\underset{S K L}{\text { Stored }}$ & 4 & $4 / 11 / 58$ & $\begin{array}{l}\text { Heat treated on Mo. Has pad marks. Used in Canada as } \\
\text { demonstrattion tube and for trial handling muns. Later } \\
\text { used for HWCTR zero power tests. }\end{array}$ \\
\hline 21 & $\mathrm{U}-2 \mathrm{w} / 0 \mathrm{Zr}$ & Th1ck wa11 & -- & $\begin{array}{l}\text { Revere } \\
3 / 11 / 58\end{array}$ & 16821 & out up & 4 & Hone & $\begin{array}{l}\text { Wandre 1 broke during extmus lon. Used for corrosion } \\
\text { studies. End seals used for core end location studies. }\end{array}$ \\
\hline 22 & $\mathrm{U}-2 \mathrm{w} / 0 \mathrm{Zr}$ & Th1ck wall & SPR-8 & $\begin{array}{l}\operatorname{ABC}(6) \\
5 / 9 / 58\end{array}$ & 18393 & Trrad1a- & 5 & $10 / 31 / 58$ & $\begin{array}{l}\text { Irradiated at SRP in low and high flux conditions. } \\
\text { Falied during high flux portion of test. }\end{array}$ \\
\hline 23 & $U-2 w / \circ 2 r$ & Th1ck wall & -- & $\begin{array}{l}A B C \\
5 / 9 / 58\end{array}$ & 18394 & Cut up & 5 & $11 / 3 / 58$ & $\begin{array}{l}\text { Used for corrosion studies, simulated spheroidizing heat } \\
\text { treatment and growth index measurements. }\end{array}$ \\
\hline 24 & $U-2 w / 0 Z F$ & Th1ck wall & -- & $5 / 23 / 58$ & 18384 & Sorapped & 5 & None & $\begin{array}{l}\text { Did not extrude because of press fallure. Billet } \\
\text { modifled and used for temperature studies. }\end{array}$ \\
\hline 25 & $\mathrm{U}-2 \mathrm{w} / \mathrm{Or}$ & Thick wal1 & -- & $6 / 27 / 58$ & 18385 & Cut up & 5 & $11 / 10 / 58$ & $\begin{array}{l}\text { Core had high carbon content, Used for corrosion } \\
\text { studies and dissolution studies. }\end{array}$ \\
\hline 26 & $\mathrm{U}-2 \mathrm{w} / \mathrm{Zr}$ & Th1ck wall & -- & $\begin{array}{l}\mathrm{ABC} \\
5 / 23 / 58\end{array}$ & 15386 & $\begin{array}{l}\text { Stored } \\
\text { : SRL }\end{array}$ & 5 & $11 / 13 / 58$ & $\begin{array}{l}\text { Originally assigned to Chalk River, Iater used for } \\
\text { HWCTR zero power studies. Sultable for lrradiation. } \\
\text { Tube No. } 72 \text {, unalloyed U, substituted for this tube for } \\
\text { Chalk River (NRU) irradiation. }\end{array}$ \\
\hline 27 & $\mathrm{U}-2 \mathrm{w} / 0 \mathrm{zr}$ & Thick wall & - & $\begin{array}{l}A B C \\
5 / 23 / 58\end{array}$ & 18387 & Cut up & 5 & None & $\begin{array}{l}\text { Serlous pick-up of } \mathrm{Zr} \text { on die during extrusion. Used to } \\
\text { check diffusion rates of copper for various heat treat- } \\
\text { ments. }\end{array}$ \\
\hline
\end{tabular}

(6) American Brass Company, Waterbury, Conn. 


\section{Summary of Nuclear Metals Power Program Fuel Tubes (Continued)}

\begin{tabular}{|c|c|c|c|c|c|c|c|c|c|}
\hline $\begin{array}{l}\text { Mu } \\
\text { Tube } \\
\text { Nor. }\end{array}$ & Compos1tion & Description & $\begin{array}{l}\text { Irrad. } \\
\text { Aasy. } \\
\text { Mumber }\end{array}$ & $\begin{array}{l}\text { Extruder } \\
\text { and Date }\end{array}$ & $\begin{array}{l}\text { NM } \\
\text { Extru. } \\
\text { Number }\end{array}$ & $\begin{array}{l}\text { Status } \\
\text { and } \\
\text { Location } \\
\end{array}$ & $\begin{array}{l}\text { Ref. } \\
\text { Info, }\end{array}$ & $\begin{array}{l}\text { Autoolave } \\
\text { Test } \\
\text { gompletion }\end{array}$ & Comments \\
\hline 28 & $\mathrm{U}-2 \mathrm{w} / \mathrm{OZr}$ & Thick wall & SPR-7 & $\begin{array}{c}A B C \\
5 / 23 / 58\end{array}$ & 18388 & $\begin{array}{l}\text { Irradia- } \\
\text { ted SRP }\end{array}$ & 5 & $111 / 17 / 58$ & $\begin{array}{l}\text { Heat treated at } 880^{\circ} \mathrm{C} \text { for } 7 \text { hours, Re-heat treated at } \\
680^{\circ} \mathrm{C} \text { for } 72 \text { hours to spheroldize epsilon. }\end{array}$ \\
\hline 29 & $\mathrm{U}-2 \mathrm{w} / 0 \mathrm{Zr}$ & Th1ck wall & SPR-4 & $6 / 27 / 58$ & 18389 & $\begin{array}{l}\text { Irrad1a- } \\
\text { ted } S R P\end{array}$ & 5 & $11 / 20 / 58$ & Falled during Irradiation at SRP \\
\hline 30 & $0-2 w / 0 \mathrm{Zr}$ & Thick wail & SPR-5 & $6 / 27 / 58$ & 13890 & $\begin{array}{l}\text { Irradia- } \\
\text { ted SRP }\end{array}$ & 5 & $111 / 24 / 58$ & None. \\
\hline 31 & $\mathrm{~J}-2 \mathrm{w} / 0 \mathrm{zr}$ & Thick wall & -- & $6 / 27 / 58$ & 18391 & $\begin{array}{c}\text { Stored } \\
\text { SRE }\end{array}$ & 5 & $11 / 28 / 58$ & $\begin{array}{l}\text { Originally assigned as spare for NRU 1rradiation. } \\
\text { Later used for HWCTR zero power tests. Sultable for } \\
\text { Irradiation. }\end{array}$ \\
\hline 32 & $\mathrm{U}-2 \mathrm{w} / \mathrm{Ozr}$ & Th1ck wall & -- & $6 / 27 / 58$ & 18392 & $\begin{array}{l}\text { Stored } \\
\text { SRL }\end{array}$ & 5 & $12 / 1 / 58$ & Used for HWCTR zero power tests. \\
\hline 33 & $\mathrm{U}-2 \mathrm{w} / 0 \mathrm{Zr}$ & $\begin{array}{l}\text { Th1ck } \\
\text { VBWR }\end{array}$ & -- & $10 / 10 / 58$ & 19657 & $\underset{\mathrm{MM}}{\text { Stored }}$ & 6 & -- & $\begin{array}{l}\text { Plekled, X-rayed, autoradiographed and heat treated. } \\
\text { Used for thermal cycling studies. }\end{array}$ \\
\hline - & $\mathrm{U}-2 \mathrm{w} / 0 \mathrm{Zr}$ & $\begin{array}{c}\text { Thick wall } \\
\text { VBWR }\end{array}$ & SPR-9 & $\begin{array}{c}A B C \\
10 / 10 / 58\end{array}$ & 19658 & $\begin{array}{l}\text { Irradia- } \\
\text { ted \$RP }\end{array}$ & 6 & $11 / 6 / 58$ & $\begin{array}{l}\text { Was not heat treated. Used by } A B C \text { as exhibition tube; } \\
\text { sent to VBWR as demonstration tube; then sent to SRP } \\
\text { for 1rradiation. Fa1led during irradiation at SRP. }\end{array}$ \\
\hline 35 & $\mathrm{U}-2 \mathrm{w} / 0 \mathrm{Zr}$ & $\begin{array}{l}\text { Th1ck wall } \\
\text { VBWR }\end{array}$ & -- & $10 / 10 / 58$ & 19659 & $\begin{array}{l}\text { Stored } \\
\text { SRL }\end{array}$ & 6 & -- & Used for flow tests at SRL. \\
\hline 36 & $\mathrm{U}-2 \mathrm{w} / 0 \mathrm{zr}$ & $\begin{array}{l}\text { Th1ck wall } \\
\text { VBWR }\end{array}$ & -- & $10 / 20 / 58$ & 19660 & $\underset{N M}{\text { Stored }}$ & 6 & -- & $\begin{array}{l}\text { P1okled, X-rayed, autoradiographed and heat treated. } \\
\text { Questionable end seal bond. }\end{array}$ \\
\hline 37 & $\mathrm{U}-2 \mathrm{w} / \mathrm{Or}$ & $\begin{array}{l}\text { Th1ck wall } \\
\text { JEWR }\end{array}$ & -- & $12 / 12 / 58$ & 19889 & $\begin{array}{l}\text { Stored } \\
\text { SRL }\end{array}$ & 6 & $1 / 4 / 59$ & $\begin{array}{l}\text { U enriched to } 3.06 \% 235 \mathrm{U} \text {. Required excessive extru- } \\
\text { sion pressure. Has questionable surface. }\end{array}$ \\
\hline 38 & $\mathrm{U}-2 \mathrm{w} / \mathrm{O} \mathrm{Zr}$ & $\begin{array}{l}\text { Th1ck wall } \\
\text { VBWR }\end{array}$ & -- & $12 / 12 / 58$ & 19890 & Cut up & 6 & None & $\begin{array}{l}\text { U enriched to } 3.06 \% 235 \mathrm{~J} \text {. Not heat treated. Destruc- } \\
\text { tively examined to obtain autoradiographic standards. } \\
\text { Also used for growth 1ndex measurements to characterize } \\
\text { tube No. } 34 \text {. }\end{array}$ \\
\hline 39 & $\mathrm{U}-2 \mathrm{w} / 0 \mathrm{zr}$ & $\begin{array}{l}\text { Ph1ck wall } \\
\text { VBWR }\end{array}$ & -- & $12 / 12 / 58$ & 19891 & $\begin{array}{l}\text { Stored } \\
\text { SRL }\end{array}$ & 6 & $1 / 26 / 59$ & $\begin{array}{l}\text { U enriched to } 3.06 \% \text { 235 } \mathrm{v} \text {. Possible candidate for } \\
\text { limited Irradiation. }\end{array}$ \\
\hline 40 & U-2 w/o zr & $\begin{array}{l}\text { Thick wall } \\
\text { VBWR }\end{array}$ & -- & $\begin{array}{c}\mathrm{ABC} \\
12 / 12 / 58\end{array}$ & 19892 & $\begin{array}{c}\text { Stored } \\
\text { SRL }\end{array}$ & 6 & $2 / 9 / 59$ & $U$ enriched to $3.06 \% 235 \mathrm{U}$. Suitable for irradiation. \\
\hline 41 & $\mathrm{U}-2 \mathrm{w} / 0 \mathrm{zr}$ & $\begin{array}{l}\text { Th1ok wall } \\
\text { VBWR }\end{array}$ & -- & $12 / 12 / 58$ & 19893 & $\begin{array}{l}\text { Irradiated } \\
\text { VBWR; } \\
\text { stored SRI }\end{array}$ & 6 & $2 / 9 / 59$ & $\begin{array}{l}\mathrm{U} \text { enriched to } 3.06 \% 235 \mathrm{U} \text {. Irradiated at VBWR to } 1200 \\
\mathrm{MWD} / \mathrm{T} \text {. }\end{array}$ \\
\hline 42 & $\mathrm{U}-2 \mathrm{w} / \mathrm{zr}$ & $\begin{array}{l}\text { Thick wall } \\
\text { VBWR }\end{array}$ & -- & $12 / 12 / 58$ & 1989 & $\begin{array}{l}\text { Stored } \\
\text { SRI }\end{array}$ & 6 & $2 / 9 / 59$ & $\begin{array}{l}\text { U enriched to } 3.06 \% 235 \text {. Formerly VBWR standby tube. } \\
\text { Sultable for lrradiation. }\end{array}$ \\
\hline
\end{tabular}

(7) VBWR - Vallec1tos Bolling Water Reactor fuel tubes. 


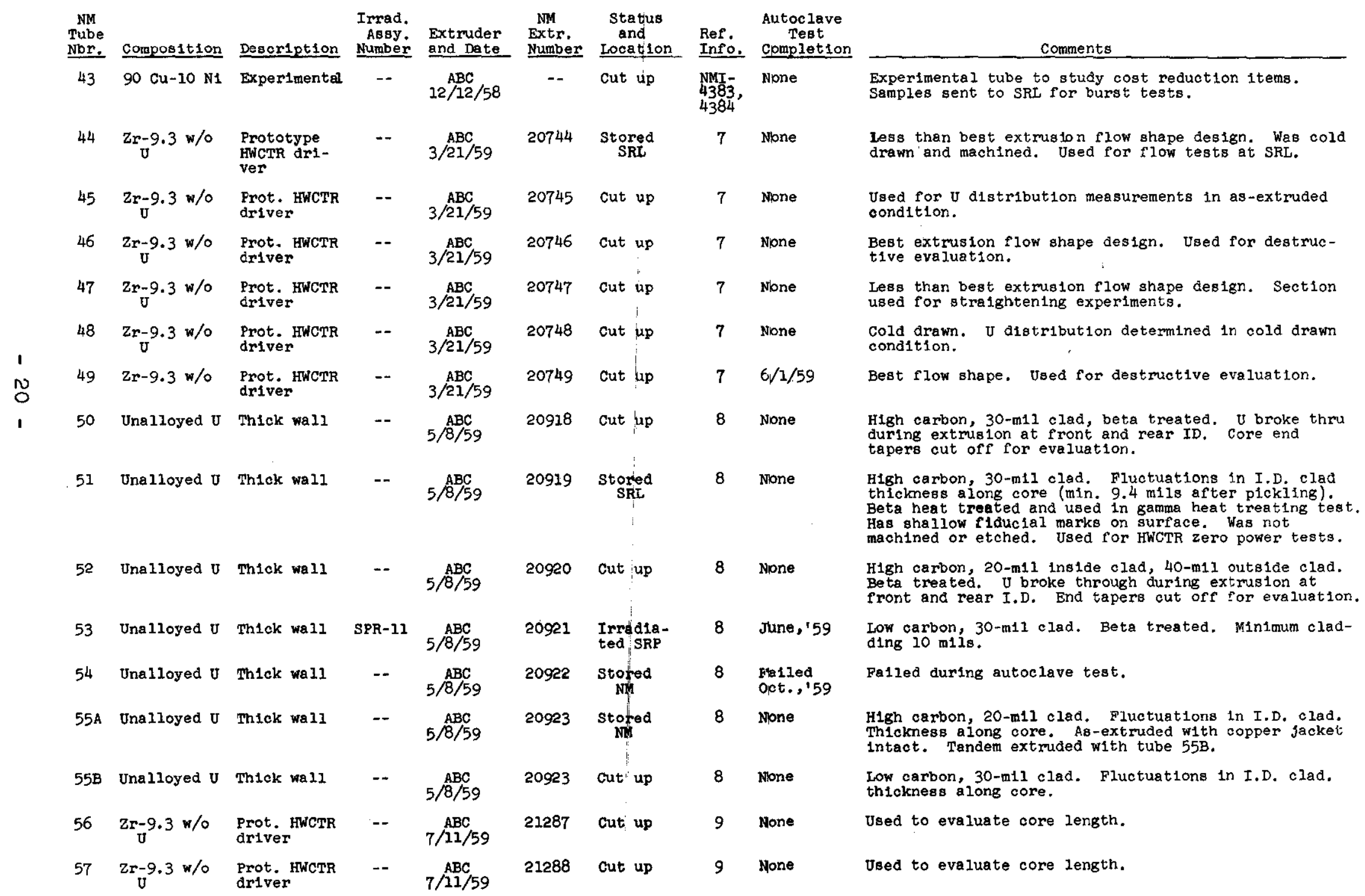




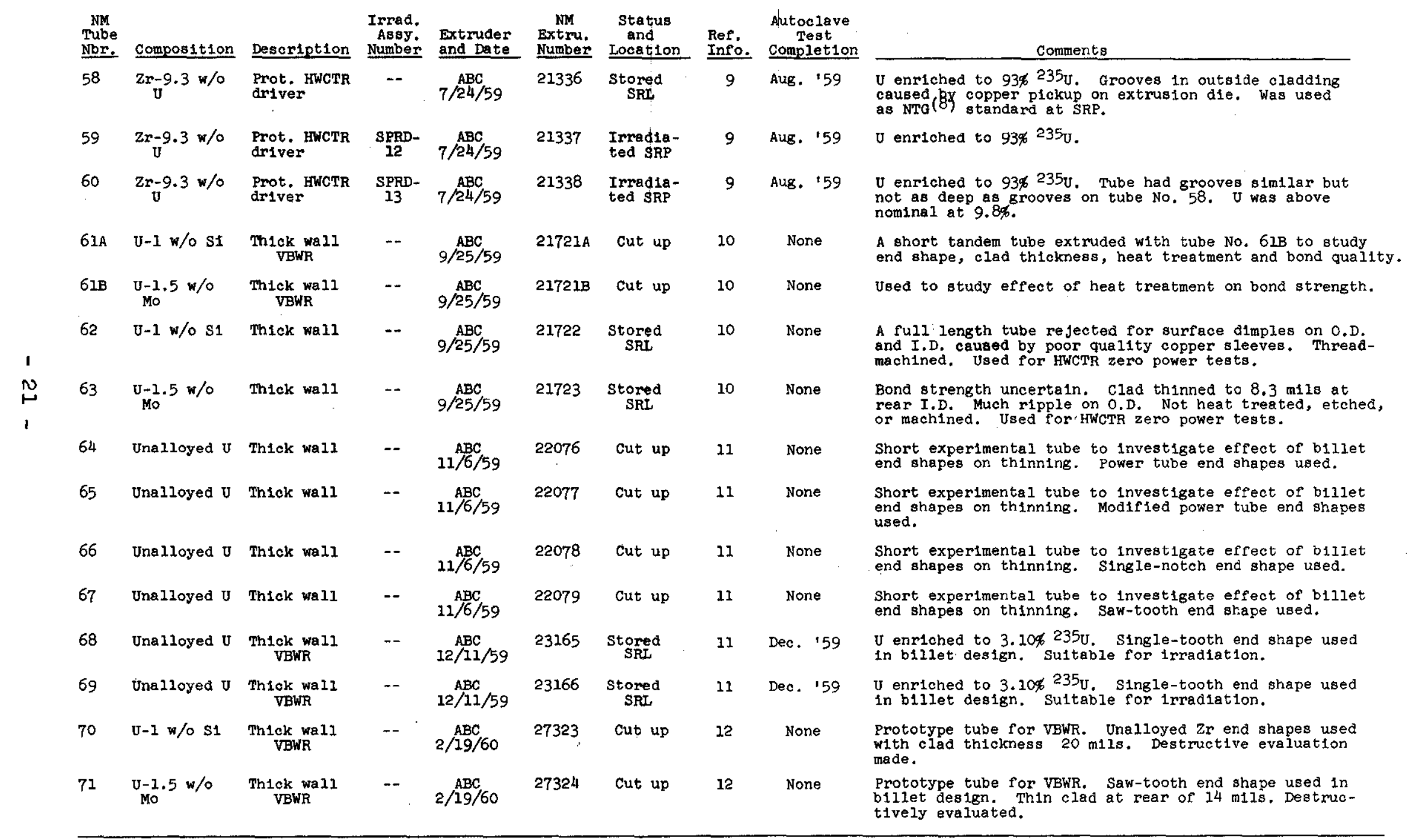

(8) NTG - Nuclear Test Gage at Savannah River Plant. 


\section{Summary of Nuclear Metals Power Program Fuel Tubes (Continued)}

\begin{tabular}{|c|c|c|c|c|c|c|c|c|c|}
\hline $\begin{array}{l}\text { NM } \\
\text { Tube } \\
\text { Mbr. }\end{array}$ & Composition & Description & $\begin{array}{l}\text { Irrad. } \\
\text { Assy. } \\
\text { Number }\end{array}$ & $\begin{array}{l}\text { Extruder } \\
\text { and Date }\end{array}$ & $\begin{array}{l}\text { MNu } \\
\text { Extru. } \\
\text { Number } \\
\end{array}$ & $\begin{array}{l}\text { Status } \\
\text { and } \\
\text { Location } \\
\end{array}$ & $\begin{array}{l}\text { Rer. } \\
\text { Info. }\end{array}$ & $\begin{array}{l}\text { Autoclave } \\
\text { Test } \\
\text { Completion }\end{array}$ & Comments \\
\hline 72 & Ona1loyed U & Th1ck wall & -- & $2 / 19 / 60$ & 27325 & $\begin{array}{l}\text { Irradia- } \\
\text { ted NRU }\end{array}$ & 13 & March 160 & $\begin{array}{l}\text { Beta treated and a1r cooled at SRL. Irradiated in } E-20 \\
\text { loop in NRU. Thin } 10-\mathrm{m} 11 \text { cladding at front } 0 . \mathrm{D} \text {. }\end{array}$ \\
\hline 73 & Unalloyed U & Th1ck wal1 & -- & $2 / 10 / 60$ & 27326 & Cut up & 13 & None & $\begin{array}{l}\text { Beta treated and a1r cooled at SRL. Inclusion in O.D. } \\
\text { cladding w1th p1t } 8 \text { mils deep. }\end{array}$ \\
\hline 74 & Unalloyed 0 & $\begin{array}{l}\text { Thin wall } \\
\text { Outer }\end{array}$ & -- & $4 / 8 / 60$ & 27944 & $\begin{array}{l}\text { Stored } \\
\text { SRL }\end{array}$ & 14 & May 160 & Not heat treated. Used for flow tests at SRL. \\
\hline $74 \mathrm{~A}$ & Unalloyed 0 & $\begin{array}{l}\text { Thin wall } \\
\text { Outer }\end{array}$ & -- & $4 / 8 / 60$ & 27746 & Cut up & 14 & None & $\begin{array}{l}\text { Modifled angular end shape used in billet. Some break- } \\
\text { through of core occurred during extrusion. }\end{array}$ \\
\hline $74 \mathrm{~B}$ & Unalloyed 0 & $\begin{array}{l}\text { Thin wall } \\
\text { Outer }\end{array}$ & -- & $4 / 8 / 60$ & 27747 & Cut up & 14 & None & Used Hanford-style spherical end shape in billet. \\
\hline 75 & Unalloyed U & $\underset{\text { Outer }}{\text { Thin }}$ & TWT $-2^{(9)}$ & $4 / 22 / 60$ & 27943 & $\begin{array}{l}\text { Irradia- } \\
\text { ted SRP }\end{array}$ & 14 & May 160 & $\begin{array}{l}\text { Beta heat treated and a1r cooled at Atlas Steels, Ltd., } \\
\text { Welland, Ontarlo. }\end{array}$ \\
\hline 76 & Unalloyed $\mathrm{U}$ & $\begin{array}{l}\text { Thin wall } \\
\text { Outer--VBwR }\end{array}$ & -- & ${ }_{4 / 22 / 60}^{N D / 2}$ & 27942 & Cut up & 14 & None & Destructively evaluated. \\
\hline 77 & $0-1 \mathrm{w} / \mathrm{os}$ & $\begin{array}{l}\text { Th1n wall } \\
\text { Outer--VBWR }\end{array}$ & -- & $4 / 23 / 60$ & 28551 & Cut up & 15 & None & $\begin{array}{l}\text { Prototype for VBWR test. Destmuctively evaluated for } \\
\text { end-seal clad thinning. }\end{array}$ \\
\hline 78 & $\mathrm{U}-1.5 \mathrm{w} / \mathrm{O}$ & $\begin{array}{l}\text { Thin wall } \\
\text { Outer--VBWR }\end{array}$ & -- & $4 / 23 / 60$ & 28552 & cut up & 15 & None & $\begin{array}{l}\text { Prototype for VBWR test. Destructively evaluated for } \\
\text { end-seal clad thinning. Also used to evaluate braze } \\
\text { seals. }\end{array}$ \\
\hline 79 & $0-1 w / 0$ S1 & $\begin{array}{l}\text { Thin wall } \\
\text { Outer--VBWR }\end{array}$ & -- & $4 / 23 / 60$ & 28553 & $\begin{array}{c}\text { Stored } \\
\text { SRL }\end{array}$ & 15 & Nay 160 & $\mathrm{U}$ enriched to $3 \phi^{235} \mathrm{U}$. Sultable for irradiation. \\
\hline 80 & $\underset{\text { Mo }}{0-1.5 w / 0}$ & $\begin{array}{l}\text { Th1n wall } \\
\text { Outer--VBWR }\end{array}$ & -- & $6 / 16 / 60$ & 28874 & Cut up & 15 & None & $\begin{array}{l}\text { VBWR prototype. Destructively evaluated for end-seal } \\
\text { clad thinning; no thinning found. Also used to } \\
\text { evaluate braze seals. }\end{array}$ \\
\hline 81 & $0-1.5 w / 0$ & $\begin{array}{l}\text { Th1n wall } \\
\text { Outer--VBWR }\end{array}$ & -- & $6 / 16 / 60$ & 28875 & Cut up & 15 & None & $\begin{array}{l}\text { VBWR prototype. Destructively evaluated with } 15-m i l \\
\text { thinning found at rear. }\end{array}$ \\
\hline 82 & $\mathrm{NO}_{\mathrm{MO}} \mathrm{-1.5 \textrm {w } / \mathrm { O }}$ & $\begin{array}{l}\text { Thin wall } \\
\text { Outer--VBWR }\end{array}$ & $\underset{(\mathbb{D})}{3 E M T-2}$ & $6 / 16 / 60$ & 29115 & $\begin{array}{l}\text { Irragla - } \\
\text { ted HicrR }\end{array}$ & 15 & May 160 & U enriched to \\
\hline 83 & $\underset{\text { Mo }}{\mathrm{O}-1.5 \% / 0}$ & $\begin{array}{l}\text { Thin wall } \\
\text { Outer--vBwR }\end{array}$ & -- & $7 / 8 / 60$ & 29116 & $\begin{array}{l}\text { Stored } \\
\text { SRI }\end{array}$ & 15 & Sept. 162 & $\mathrm{U}$ enriched to $3 x^{235}$. Sultable for irradiation. \\
\hline 84 & Unalloyed U & $\begin{array}{l}\text { Th1n wall } \\
\text { Outer }\end{array}$ & -- & $\begin{array}{c}\text { MM } \\
\text { June' } 60\end{array}$ & 28137 & Cut up & 16 & None & $\begin{array}{l}\text { Billet design used } 10^{\circ} \text { core bevels plus other preshap- } \\
\text { ing with larger angles. }\end{array}$ \\
\hline 85 & Unalloyed U & $\begin{array}{l}\text { Thin wall } \\
\text { Outer }\end{array}$ & -- & May 60 & 28133 & Cut up & 16 & None & $\begin{array}{l}\text { Billet design used stepped end shape. Portions of tube } \\
\text { used for transient melting studies. }\end{array}$ \\
\hline 86 & Unalloyed U & $\begin{array}{l}\text { Thin wall } \\
\text { Outer }\end{array}$ & - & $\underset{\text { May' }}{\mathrm{MM}} 60$ & 28136 & Cut up & 16 & None & Similar to tube No. 84 . \\
\hline
\end{tabular}

(9) SRP number, TWT - thin-waIled tube.

(10) HWCTR number, ENT - enriched metal tube. 


\begin{tabular}{|c|c|c|c|c|c|c|c|c|c|c|}
\hline $\begin{array}{l}\text { NM } \\
\text { Tube } \\
\text { Nor. }\end{array}$ & Compos1tion & Description & $\begin{array}{l}\text { Irrad. } \\
\text { Assy. } \\
\text { Number }\end{array}$ & $\begin{array}{l}\text { Extmuder } \\
\text { and Dete }\end{array}$ & $\begin{array}{l}\text { NM } \\
\text { Extmu. } \\
\text { Number }\end{array}$ & $\begin{array}{r}\text { St: } \\
\text { ax } \\
\text { Loce }\end{array}$ & ind & $\begin{array}{l}\text { Ref. } \\
\text { Info. }\end{array}$ & $\begin{array}{l}\text { Autoclave } \\
\text { Test } \\
\text { Completion }\end{array}$ & Comments \\
\hline 87 & Unalloyed t & $\begin{array}{c}\text { Thin wall } \\
\text { Outer }\end{array}$ & -- & May 160 & 28134 & cut & up & 16 & None & B1llet design used uncompensated radius. \\
\hline 88 & Unalloyed U & $\begin{array}{l}\text { Thin wall } \\
\text { Outer }\end{array}$ & -- & $\begin{array}{ll}\mathrm{NM} \\
\mathrm{July} & 60\end{array}$ & 28138 & cut & & 16 & None & $\begin{array}{l}\text { Billet dealgn used tapered inner sleeve at rear with } \\
\text { front the same as tube No. } 84 \text {. }\end{array}$ \\
\hline 89 & Unalloyed 0 & $\begin{array}{l}\text { Thin wall } \\
\text { Outer }\end{array}$ & $\cdots$ & July'60 & 28140 & cut & up & 16 & None & Billet design used short $10^{\circ}$ core bevels. \\
\hline 90 & Unalloyed U & $\begin{array}{c}\text { Thin wail } \\
\text { Outer }\end{array}$ & -- & ${ }_{\text {May }}^{\mathrm{NM}}+60$ & 28127 & cut & up & 16 & None & Billet design used flat $0^{\circ}$ compensation. \\
\hline 91 & Jnalloyed U & $\begin{array}{l}\text { Thin wall } \\
\text { Outer }\end{array}$ & -- & May 160 & 28130 & cut & up & 16 & None & Billet deslgn used flat $+26^{\circ}$ compensation. \\
\hline 92 & Unalloyed U & $\begin{array}{l}\text { Thin wall } \\
\text { Outer }\end{array}$ & -- & $\operatorname{may}^{\text {NM }} 160$ & 28132 & cut & up & 16 & None & Billet design used flat $45^{\circ}$ compensation. \\
\hline 93 & Unalloyed 0 & $\begin{array}{c}\text { Th1n wall } \\
\text { Outer }\end{array}$ & -- & May 160 & 28129 & cut & up & 16 & None & Billet design used flat $-15^{\circ}$ compensation. \\
\hline$\stackrel{N}{\omega}$ & Una1loyed U & $\begin{array}{l}\text { Thin wall } \\
\text { Outer }\end{array}$ & -- & $\mathrm{May} \cdot 60$ & 28126 & Cut & up & 16 & None & Billet design used flat $-27^{\circ}$ compensation. \\
\hline 95 & Unalloyed U & $\begin{array}{l}\text { Th1n wall } \\
\text { Outer }\end{array}$ & -- & $6 / 18 / 60$ & 28965 & $\begin{array}{r}\text { Stor } \\
\text { SF }\end{array}$ & & 16 & Nov. 160 & $\begin{array}{l}\text { 011-quenched at SRL; a 1r cooled, beta treated at Atlas } \\
\text { on } 10 / 4 / 60 \text {. Flow tested at SRL. }\end{array}$ \\
\hline 96 & Una1 loyed U & $\begin{array}{l}\text { Thin wall } \\
\text { Outer }\end{array}$ & -- & $\frac{N M}{6 / 28 / 60}$ & 28966 & $\begin{array}{l}\text { Stor } \\
\text { NM }\end{array}$ & & 16 & None & $\begin{array}{l}\text { Billet design same as tube No. } 84 \text {. Beta treated and } \\
\text { a1r cooled at SRL. Damaged during extrusion press } \\
\text { failure. Stored in as-plckled condition. }\end{array}$ \\
\hline 97 & Unalloyed U & $\begin{array}{l}\text { Thin wall } \\
\text { Outer }\end{array}$ & -- & $6 / 18 / 60$ & 28967 & $\begin{array}{r}\text { Stor } \\
\text { SR }\end{array}$ & red & 16 & Nov. ${ }^{160}$ & $\begin{array}{l}\text { 011-quenched at SRL; air cooled, beta treated at Atlas } \\
\text { on } 10 / 4 / 60 \text {. Flow tested at SRL. }\end{array}$ \\
\hline 98 & Unalloyed U & $\begin{array}{l}\text { Thin wall } \\
\text { outer- VBWR }\end{array}$ & -- & $6 / 18 / 60$ & 28968 & Cut & up & 16 & None & $\begin{array}{l}\text { VBWR prototype. B1llet design used long } 10^{\circ} \text { core } \\
\text { bevels. }\end{array}$ \\
\hline 99 & Unalloyed U & $\begin{array}{l}\text { Thin wall } \\
\text { Outer }\end{array}$ & -- & July'60 & 28142 & Cut & up & 16 & None & Billet design used long $10^{\circ}$ core bevels. \\
\hline 100 & Unalloyed U & $\begin{array}{l}\text { Thin wall } \\
\text { Inner }\end{array}$ & -- & $\stackrel{\text { NMI }}{\text { June: } 60}$ & 29205 & Cut & up & 17 & None & Destmuctively evaluated for end-seal clad-thinning. \\
\hline 101 & Unalloyed 0 & $\begin{array}{l}\text { Thin wall } \\
\text { Inner }\end{array}$ & -- & $\underset{\text { June }}{\text { NMM }} 60$ & 29206 & Cut & up & 17 & None & $\begin{array}{l}\text { Destructively evaluted for end-seal clad-thinning. } \\
\text { B1llet des1gn used } 10 \text { core bevels. Sections used for } \\
\text { m1crostructure analys1s at SRL. }\end{array}$ \\
\hline 102 & Unallojed U & $\begin{array}{c}\text { Th1n wall } \\
\text { Inner }\end{array}$ & -- & Nune 160 & 29207 & cut & up & 17 & None & Destructively evaluated for end-seal clad-thinning. \\
\hline 103 & Unalloyed 0 & $\begin{array}{l}\text { Thin wall } \\
\text { Inner }\end{array}$ & TWIT-3 & $8 / 5 / 60$ & 29309 & $\begin{array}{l}\text { Irra } \\
\text { ted }\end{array}$ & SRP & 17 & oct. 60 & A1r-cooled at Atlas on $10 / 4 / 60$ \\
\hline 104 & Unalloyed 0 & $\begin{array}{c}\text { Th1n wall } \\
\text { Innex }\end{array}$ & TWIT-3 & $8 / 5 / 60$ & 29310 & $\begin{array}{l}\text { Irra } \\
\text { ted }\end{array}$ & $\begin{array}{l}\text { ad1e- } \\
\text { SRP }\end{array}$ & 17 & oct. 160 & Air-cooled at Atlas on $10 / 4 / 60$ \\
\hline
\end{tabular}

(11) SRP number, TWIT - thin-walled Inner tube. 
Summary of Nuclear Metals Power Program Fuel Tubes (Continued)

\begin{tabular}{|c|c|c|c|c|c|c|c|c|c|}
\hline $\begin{array}{l}\text { MM } \\
\text { Tube } \\
\text { Nor. }\end{array}$ & Composition & Description & $\begin{array}{l}\text { Irrad. } \\
\text { Assy. } \\
\text { Number }\end{array}$ & $\begin{array}{l}\text { Extmuder } \\
\text { and pate }\end{array}$ & $\begin{array}{l}\text { NM } \\
\text { Extmu. } \\
\text { Number }\end{array}$ & $\begin{array}{l}\text { Status } \\
\text { snd } \\
\text { Location } \\
\end{array}$ & $\begin{array}{l}\text { Ref. } \\
\text { Info. }\end{array}$ & $\begin{array}{l}\text { Autoclave } \\
\quad \text { Test } \\
\text { Completion }\end{array}$ & Comments \\
\hline 105 & Unalloyed U & $\begin{array}{l}\text { Thin wa11 } \\
\text { Inner }\end{array}$ & $\cdots$ & $8 / 5 / 60$ & 29311 & $\begin{array}{l}\text { Stored } \\
\text { SRL }\end{array}$ & 17 & None & Not heat treated. Used for flow tests at SRL. \\
\hline 106 & Unalloyed 0 & $\begin{array}{l}\text { Thin wall } \\
\text { Outer- VBWR }\end{array}$ & -- & $8 / 30 / 60$ & 29589 & Cut up & 16 & None & VBWR prototype. Thinning at front ID. \\
\hline 107 & Unalloyed 0 & $\begin{array}{l}\text { Thin wall } \\
\text { Outer }\end{array}$ & -- & $9 / 2 / 60$ & 29593 & Cut up & 18 & None & $\begin{array}{l}\text { Destructive evaluation showed good centering of front } \\
\text { taper. cladding uniform w1th } 20 \text { mils minimum. }\end{array}$ \\
\hline 108 & Unalioyed U & $\begin{array}{l}\text { Th1n wall } \\
\text { Outer }\end{array}$ & $\min -3$ & $9 / 23 / 60$ & 29778 & $\begin{array}{l}\text { Irradiabed } \\
\text { SRP }\end{array}$ & 18 & Nov. 160 & Air cooled at Atlas on $10 / 4 / 60$. \\
\hline 109 & Unalloyed U & $\begin{array}{l}\text { Thin mall } \\
\text { Outer }\end{array}$ & -- & $9 / 23 / 60$ & 29779 & $\underset{\mathrm{NM}}{\text { Stored at }}$ & 18 & Nov. 160 & $\begin{array}{l}\text { Air cooled at Atlas on } 10 / 4 / 60 \text {. Very 1rregular cladding } \\
\text { as indicated by eddy current examination. Stored in } \\
\text { autoclaved condition. }\end{array}$ \\
\hline 110 & Unalloyed $v$ & $\begin{array}{l}\text { Thin wall } \\
\text { Outer }\end{array}$ & -- & $9 / 23 / 60$ & 29780 & Stored & 18 & Nov, 160 & Air cooled at Atlas on $10 / 4 / 60$. \\
\hline 131 & Unalloyed U & $\begin{array}{l}\text { Thin wall } \\
\text { Outer }\end{array}$ & -- & $9 / 23 / 60$ & 29781 & $\begin{array}{l}\text { Stored } \\
\text { SRL }\end{array}$ & 18 & Nov. 160 & $\begin{array}{l}\text { Breakthrough during 'extrusion. A1r cooled at Atlas on } \\
10 / 4 / 60 \text {. Used for flow tests at SRL. }\end{array}$ \\
\hline 112 & Unalloyed U & $\begin{array}{l}\text { Th1n wall } \\
\text { Outer }\end{array}$ & -- & $9 / 23 / 60$ & 29782 & $\underset{N N}{\text { Stored }}$ & 18 & Nov. 160 & $\begin{array}{l}\text { A1r cooled at Atlas on } 10 / 4 / 60 \text {. Double-autoclaved. } \\
\text { Used for autoclave stra1ghtening tests. }\end{array}$ \\
\hline 113 & Unalloyed U & $\begin{array}{l}\text { Thin wa11 } \\
\text { Outer- VBWR }\end{array}$ & -- & $9 / 29 / 60$ & 29861 & cut up & 16 & None & VBWR prototype. \\
\hline 114 & Unalloyed U & $\begin{array}{l}\text { Thin wall } \\
\text { Outer- VBWR }\end{array}$ & -- & $10 / 19 / 60$ & 30103 & $\begin{array}{l}\text { Stored } \\
\text { SRI }\end{array}$ & 19 & Nov. 160 & $\begin{array}{l}\text { U enriched to } 3 x^{235} \text {. Some shallow depression less } \\
\text { than } 3 \text { mils. Suitable for 1rradiation. }\end{array}$ \\
\hline 115 & Unalloyed 0 & $\begin{array}{l}\text { Thin wall } \\
\text { Outer- VBWR }\end{array}$ & -- & $10 / 19 / 60$ & 30104 & $\begin{array}{c}\text { Stored } \\
\mathrm{SRL}\end{array}$ & 19 & Nov, 160 & $\begin{array}{l}\text { U enriched to } 3 \% 235 \text {. Some shallow depressions. } \\
\text { Sultable for irradiation. }\end{array}$ \\
\hline 116 & Unalloyed $v$ & $\begin{array}{l}\text { Then wall } \\
\text { Inner }\end{array}$ & -- & $12 / 2 / 60$ & 30795 & $\underset{N M}{\text { Stored }}$ & 20 & None & Inner tube demonstration set. Surface scratches on ID. \\
\hline 117 & Unalloyed U & $\begin{array}{c}\text { Thin wall } \\
\text { Inner }\end{array}$ & TWIT-2 & $12 / 2 / 60$ & 30796 & $\begin{array}{l}\text { Irrad1ated } \\
\text { SRP }\end{array}$ & 20 & $\operatorname{Jan} .161$ & Inner tube demonstration set. \\
\hline 128 & Unalloyed U & $\begin{array}{l}\text { Thin wall } \\
\text { Inner }\end{array}$ & -- & $\begin{array}{c}\mathrm{MM} \\
12 / 2 / 60\end{array}$ & 30797 & $\begin{array}{c}\text { Stored } \\
N M\end{array}$ & 20 & None & Inner tube demonstration set. Surface scratches on ID. \\
\hline 119 & Onalloyed U & $\begin{array}{c}\text { Thin wali } \\
\text { Inner }\end{array}$ & TWIT-2 & $12 / 2 / 60$ & 30798 & $\begin{array}{c}\text { Irrad1ated } \\
\text { SRP }\end{array}$ & 20 & Jan. 161 & Inner tube demonstration set. \\
\hline 120 & Unalloyed U & $\begin{array}{l}\text { Thin wall } \\
\text { Inner }\end{array}$ & -- & $12 / 2 / 60$ & 30799 & $\begin{array}{c}\text { stored } \\
\text { SRL }\end{array}$ & 20 & None & Inner tube demonstration set. UBed for flow tests at SRL. \\
\hline 121 & Unalloyed U & $\underset{\text { Inner }}{\text { Thin wall }}$ & -- & $12 / 2 / 60$ & 30800 & $\begin{array}{l}\text { Stored } \\
\text { SRL. }\end{array}$ & 20 & Deo. 160 & Not heat treated. Uned for flow tes ts at SRL. \\
\hline
\end{tabular}




\begin{tabular}{|c|c|c|c|c|c|c|c|c|c|}
\hline $\begin{array}{l}\text { NRI } \\
\text { Tube } \\
\text { Nar. }\end{array}$ & Composition & Description & $\begin{array}{l}\text { Irrad. } \\
\text { Assy. } \\
\text { Number }\end{array}$ & $\begin{array}{l}\text { Extruder } \\
\text { and Date }\end{array}$ & $\begin{array}{l}\text { Nu } \\
\text { Extru. } \\
\text { Number }\end{array}$ & $\begin{array}{l}\text { Status } \\
\text { and } \\
\text { Location } \\
\end{array}$ & $\begin{array}{l}\text { Ref. } \\
\text { Info. }\end{array}$ & $\begin{array}{l}\text { Autoclave } \\
\text { Test } \\
\text { Completion } \\
\end{array}$ & Comments \\
\hline 122 & Unalloyed U & $\begin{array}{c}\text { Thin wall } \\
\text { Outer }\end{array}$ & $=$ & $\underset{2 / 24 / 61}{\mathrm{NM}}$ & 31229 & Cut up & 21. & None & $\begin{array}{l}\text { Experimental tube to improve core-clad interface. Core } \\
\text { prepared by upset-extrusion of triple beta treated } \\
\text { casting. Marked improvement noted. }\end{array}$ \\
\hline 123 & Onalloyed $\sigma$ & $\begin{array}{l}\text { Thin wall } \\
\text { Outer }\end{array}$ & - & $2 / 24 / 61$ & 31230 & Cut up & 21 & None & $\begin{array}{l}\text { Experimental tube to Improve core-clad interface using } \\
\text { a chilled casting with core processing similar to } \\
\text { tube No. 12. Se. Severe shift caused by ram-mandrel mis- } \\
\text { aligninent. }\end{array}$ \\
\hline 124 & Enalloyed $\mathrm{J}$ & $\begin{array}{l}\text { Thin wall } \\
\text { Outer }\end{array}$ & -- & $5 / 4 / 61$ & 31873 & Cut up & 21 & None & Evaluation of solid $\mathrm{zr}$ billet nose. \\
\hline 125 & Unalloyed 0 & $\begin{array}{l}\text { Thin wali } \\
\text { Outer }\end{array}$ & -- & $5 / 18 / 61$ & 32001 & $\underset{\text { MM }}{\text { Stored }}$ & 22 & July :61 & HWCTR outer tube prototype. \\
\hline 126 & Unalloyed U & $\begin{array}{l}\text { Thin wall } \\
\text { Outer }\end{array}$ & -- & $\stackrel{\operatorname{MM}}{5 / 28 / 61}$ & 32002 & $\underset{\text { Stop }}{\text { Stored }}$ & 22 & July 161 & HWCTR outer tube prototype. \\
\hline 127 & Unalloyed 0 & $\underset{\text { Outer }}{\text { Th1n wall }}$ & - & $5 / 18 / 61$ & 32003 & $\underset{\text { Stored }}{\text { NM }}$ & 22 & July 161 & HWCTR outer tube prototype. \\
\hline 128 & Unalloyed 0 & $\begin{array}{l}\text { Thin wall } \\
\text { Inner }\end{array}$ & -- & $5 / 26 / 61$ & 32039 & $\underset{N M}{\text { Stored }}$ & 23 & Aug. 161 & $\begin{array}{l}\text { Type D restraint tube with } 60 \mathrm{mll} \text { cladding both } \mathrm{OD} \text { and ID } \\
5-8 \text { m11 striation in cladding. }\end{array}$ \\
\hline 129 & Onalloyed 0 & $\underset{\text { Thin ral1 }}{\text { Inner }}$ & -- & $\underset{5 / 26 / 61}{\operatorname{Mng}}$ & 32040 & $\begin{array}{r}\text { Stored } \\
\text { SRL }\end{array}$ & 23 & Aug. 161 & $\begin{array}{l}\text { Type E restraint tube with } 40 \mathrm{~m} 1 \mathrm{l} \text { OD clad and } 25 \mathrm{~m} 1 \mathrm{ID} \\
\text { clad. Sultable for Irradiation. }\end{array}$ \\
\hline 130 & Unalloyed $\mathrm{J}$ & $\underset{\text { Thiter }}{\text { Thall }}$ & -- & $6 / 15 / 61$ & 32345 & $\begin{array}{r}\text { Stored } \\
\text { SRL }\end{array}$ & 24 & Aug. 161 & $\begin{array}{l}\text { Type B restraint tube with } 25 \mathrm{mil} \text { oD clad and } 40 \mathrm{mll} \text { ID } \\
\text { clad. Sultable for Irradiation. }\end{array}$ \\
\hline 131 & Unalloyed $\mathrm{J}$ & $\begin{array}{l}\text { Thin wall } \\
\text { Outer }\end{array}$ & -- & $6 / 15 / 61$ & 32346 & $\begin{array}{r}\text { Stored } \\
\text { SRL }\end{array}$ & 24 & Aug. 161 & $\begin{array}{l}\text { Type } c \text { restraint tube with } 40 \mathrm{~m} 11 \text { OD } \mathrm{clad} \text { and } 25 \mathrm{~m} 1 \mathrm{ID} \\
\text { clad. Suitable for } 1 \text { rradiation. }\end{array}$ \\
\hline 132 & Enalloyed 0 & $\begin{array}{l}\text { Th1n wall } \\
\text { Outer }\end{array}$ & -- & $\begin{array}{l}A B C \\
8 / 25 / 61\end{array}$ & 32995 & $\underset{m}{\text { Stored }}$ & 24 & Oct. 161 & $\begin{array}{l}\text { Restraint tube with } 60 \mathrm{~m} 11 \text { oD and ID cladding. Slight } \\
\text { wh1te oxide over } 2 \mathrm{ft} \text {. span caused by autoclave } \\
\text { malfunction. }\end{array}$ \\
\hline 133 & Enalloyed $\mathrm{J}$ & $\underset{\text { Outer }}{\text { Thin wall }}$ & $\begin{array}{l}\text { RMT }-1-2 \\
(12)\end{array}$ & $8 / 25 / 61$ & 32996 & $\begin{array}{l}\text { Irradiated } \\
\text { BwCrR }\end{array}$ & 24 & $\operatorname{Jan} .162$ & Type A restraint tube with $60 \mathrm{~m} 11 \mathrm{~s}$ oD and ID cladding. \\
\hline 134 & $\nabla-1 \approx / 0$ S1 & $\begin{array}{l}\text { Th1n wall } \\
\text { Outer }\end{array}$ & -- & $8 / 25 / 61$ & 32997 & Sorapped & 25 & None & Experimental alloy tube. Stalled in extrusion press. \\
\hline 135 & $\begin{array}{l}0-0.3 \mathrm{w} / 0 \mathrm{Al} \\
-0.5 \mathrm{w} / \mathrm{S}\end{array}$ & $\begin{array}{l}\text { Th1n wall } \\
\text { Outer }\end{array}$ & -- & $8 / 25 / 61$ & 32998 & $\begin{array}{r}\text { Stored } \\
8 R L\end{array}$ & 25 & Jan. 162 & $\begin{array}{l}\text { Experimental ternary alloy tube. Sultable for } \\
\text { Irradiation. }\end{array}$ \\
\hline 136 & Unalloyed E & $\begin{array}{l}\text { Thin weil } \\
\text { Inner }\end{array}$ & -- & $9 / 9 / 61$ & 33158 & $\underset{\text { SRL }}{\text { Stored }}$ & 23 & Jan. 162 & $\begin{array}{l}\text { Type D restraint tube w1th } 60 \mathrm{~m} 11 \mathrm{~s} \text { oD and ID cladding. } \\
\text { Replacement for tube No. } 128 \text {. Sultable for Irradiation. }\end{array}$ \\
\hline
\end{tabular}

(12) HWCYR number, RMr - Restraint metal tube. 
Summary of Nuclear Metals Power Program Fuel Tubes (Continued)

\begin{tabular}{|c|c|c|c|c|c|c|c|c|c|}
\hline $\begin{array}{l}\text { Mri } \\
\text { Trube } \\
\text { Nbr. }\end{array}$ & Composition & Description & $\begin{array}{l}\text { Irrad. } \\
\text { Assy. } \\
\text { Number }\end{array}$ & $\begin{array}{l}\text { Extmuder } \\
\text { and Date }\end{array}$ & $\begin{array}{l}\text { Mar } \\
\text { Extru. } \\
\text { Number }\end{array}$ & $\begin{array}{l}\text { Status } \\
\text { and } \\
\text { Location } \\
\end{array}$ & $\begin{array}{l}\text { Ref. } \\
\text { Info. }\end{array}$ & $\begin{array}{l}\text { Autoolave } \\
\text { Test } \\
\text { Completion } \\
\end{array}$ & Comments \\
\hline 137 & Unalloyed $\mathrm{U}$ & $\begin{array}{l}\text { Thin wall } \\
\text { Inner }\end{array}$ & -- & $\begin{array}{c}\mathrm{NM} \\
2 / 14 / 62\end{array}$ & -- & Cut up & 26 & None & $\begin{array}{l}\text { Prototype for HWCTR enriched tubes for scouting billet } \\
\text { design parameters and to evaluate non bond standards. }\end{array}$ \\
\hline 138 & Unalloged 0 & $\begin{array}{l}\text { Thin wall } \\
\text { Outer }\end{array}$ & -- & $\stackrel{M M}{M / 8 / 62}$ & 35820 & Gut up & 27 & None & Demonstration of Process B core preparation. \\
\hline 139 & Unalloyed 0 & $\underset{\text { Outer }}{\text { Thin wall }}$ & -- & $\stackrel{\operatorname{MN}}{3 / 8 / 62}$ & 35821 & Cut up & 27 & None & Demonstration of Process B core preparation. \\
\hline 240 & Unallojed 0 & $\begin{array}{l}\text { Thin wall } \\
\text { Outer }\end{array}$ & -- & $3 / 8 / 62$ & 35819 & Cut up & 27 & None & Demonstration of Process $B$ core preparation. \\
\hline 141 & v-1 w/o s1 & $\begin{array}{l}\text { Thin wall } \\
\text { Outer }\end{array}$ & -- & $\stackrel{A B C}{4 / 14 / 62}$ & 35897 & $\begin{array}{l}\text { Stored } \\
\text { SRI }\end{array}$ & 25 & June 162 & Experimental alloy tube. Suftable for Irradiation. \\
\hline 142 & U-1.5 x/0 Mo & $\underset{\text { Outer }}{\text { Thin wall }}$ & $=$ & $4 / 14 / 62$ & -- & $\underset{\text { SM }}{\text { Stored }}$ & 25 & None & $\begin{array}{l}\text { Experimental alloy tube. Stored in as-extruded } \\
\text { condition. }\end{array}$ \\
\hline 143 & Unalloyed 0 & $\begin{array}{l}\text { Thin wall } \\
\text { Inner }\end{array}$ & $\cdots$ & $4 / 27 / 62$ & 35929 & $\begin{array}{r}\text { Stored } \\
\text { SRI }\end{array}$ & 26 & June 162 & $\begin{array}{l}\text { U enriched to } 2.1 \% \text { 235v } 011 \text { quenched at SRL.) Suit- } \\
\text { able for Irradiation. ("No.1" marked on tube.) }\end{array}$ \\
\hline $\begin{array}{l}n \\
\text { a } \\
1\end{array}$ & Onalloyed $\theta$ & $\begin{array}{l}\text { Thin wall } \\
\text { Inner }\end{array}$ & -- & $4 / 27 / 62$ & 35930 & $\begin{array}{l}\text { Stored } \\
\text { SRI }\end{array}$ & 26 & June 162 & 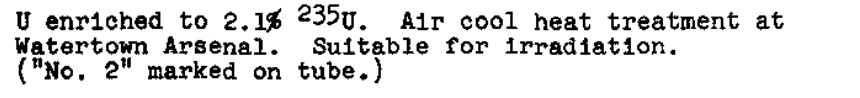 \\
\hline 145 & Onalloyed 0 & $\begin{array}{l}\text { Thin wall } \\
\text { Inner }\end{array}$ & -- & $4 / 27 / 62$ & 35931 & $\begin{array}{c}\text { Stored } \\
\text { SRL }\end{array}$ & 26 & Nov. 162 & $\begin{array}{l}\text { U enrlehed to } 2.1 \% 235 \mathrm{~J} \text {. } 011 \text { quenched at SRL. } \\
\text { Sultable for 1rradiation. }\end{array}$ \\
\hline 146 & Enalloyed U & $\begin{array}{l}\text { Thin wall } \\
\text { Inner }\end{array}$ & -- & $4 / 27 / 62$ & 35932 & $\begin{array}{c}\text { Stored } \\
\text { SRI }\end{array}$ & 26 & Nov. 162 & $\begin{array}{l}\text { U enriched to } 2.1 \% 235 \mathrm{~J}, 011 \text { quenched at SRL. Trregular } \\
\text { cladding th1ckness. Sultable for limited 1rradiation. }\end{array}$ \\
\hline 147 & Unalloyed U & $\begin{array}{l}\text { Thin wall } \\
\text { Inner }\end{array}$ & -- & $\underset{4 / 27 / 62}{M M}$ & 35933 & $\begin{array}{l}\text { Stored } \\
\text { SRI }\end{array}$ & 26 & June 162 & $\begin{array}{l}\text { U enriched to } 2.1 \%{ }^{235} \mathrm{v} \text {. } 011 \text { quenched at SRL. Sultable } \\
\text { for 1rrad1ation. (" } 5 \text { " marked on tube.) }\end{array}$ \\
\hline 148 & Unalloyed U & $\begin{array}{c}\text { Thin wall } \\
\text { Outer }\end{array}$ & $\frac{\text { ETwO-2 }}{(13)}$ & $6 / 29 / 62$ & 36308 & $\begin{array}{l}\text { Irradiated } \\
\text { HWCTR }\end{array}$ & 28 & Jan. 163 & $\mathrm{U}$ enriched to $2.1 \% 235 \mathrm{v}$. 011 quenched at SRL. \\
\hline 149 & Unalloyed U & $\begin{array}{l}\text { Thin wall } \\
\text { Outer }\end{array}$ & EYWO-3 & $6 / 29 / 62$ & 36309 & $\begin{array}{c}\text { Irradiated } \\
\text { HWCIR }\end{array}$ & 28 & Jan. 163 & U enrlched to $2.16235 \mathrm{~J}$. 011 quenched at SRL. \\
\hline 150 & Unalloyed 0 & $\begin{array}{l}\text { Short } \\
\text { HWCHR }\end{array}$ & -- & $11 / 5 / 62$ & 36692 & Cut up & 29 & None & Braze development. \\
\hline 151 & Unalloged V & $\begin{array}{l}\text { Short } \\
\text { HWCWR }\end{array}$ & -- & $11 / 5 / 62$ & 36693 & $\begin{array}{c}\text { Stored } \\
\text { SRI }\end{array}$ & 29 & March 163 & $\begin{array}{l}\text { Tube cut into } 11 \frac{1}{4} \text { sections with end caps brazed. Six } \\
\text { sections sent to SRL as 1rradiation oandidates. }\end{array}$ \\
\hline 152 & $\begin{array}{l}\mathrm{J}-350 \mathrm{Fe}-900 \\
\mathrm{A1}\end{array}$ & $\begin{array}{l}\text { Short } \\
\text { HWGrR }\end{array}$ & -- & $12 / 26 / 62$ & 36823 & Cut up & 29 & None & Braze development. \\
\hline
\end{tabular}

(13) HWCRR number, ENo - Enriched thin wall outer tube. 
Summary of Nuclear Metals Power Program Fuel Tubes (Continued)

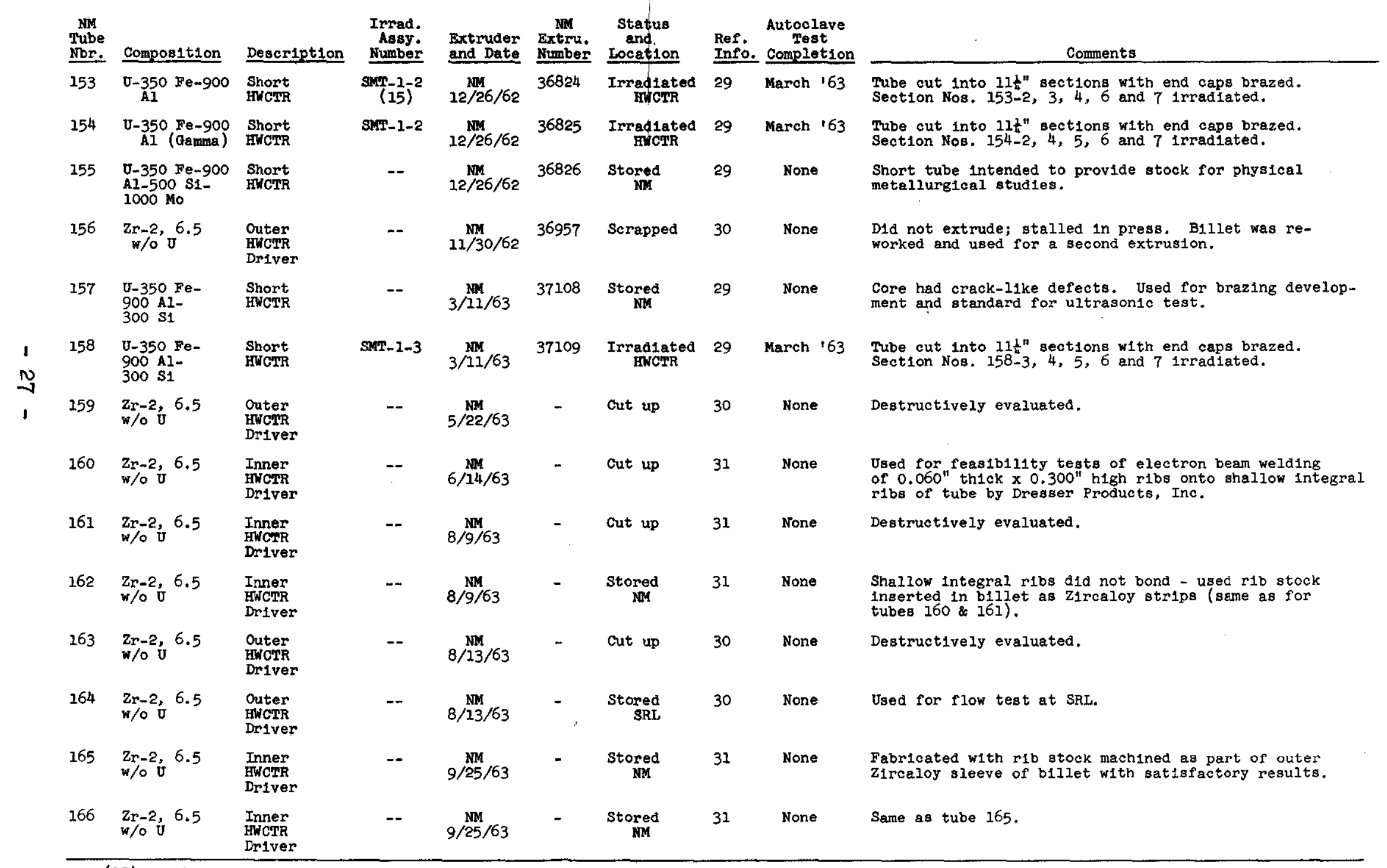

(15) HWCTR number, SMT - Segmented metal tube. 
Summary of Nuclear Metals Power Program Fuel Tubes (Continued)

\begin{tabular}{|c|c|c|c|c|c|c|c|c|c|}
\hline $\begin{array}{l}\text { NM } \\
\text { Tube } \\
\text { Nbr. }\end{array}$ & Composition & Description & $\begin{array}{l}\text { Irrad. } \\
\text { Assy. } \\
\text { Number } \\
\end{array}$ & $\begin{array}{l}\text { Extruder } \\
\text { and Date }\end{array}$ & $\begin{array}{l}\text { NM } \\
\text { Extru. } \\
\text { Number }\end{array}$ & $\begin{array}{l}\text { Status } \\
\text { and } \\
\text { Location } \\
\end{array}$ & $\begin{array}{l}\text { Ref. } \\
\text { Info. }\end{array}$ & $\begin{array}{l}\text { Autoclave } \\
\text { Test } \\
\text { Completion } \\
\end{array}$ & Comments \\
\hline 167 & $\underset{\mathrm{w} / \mathrm{O} \text { U }}{\mathrm{Zr}-2}, 11.1$ & $\begin{array}{l}\text { Single } \\
\text { HWCTR } \\
\text { Driver }\end{array}$ & -- & $\frac{\mathrm{NM}}{11 / 6 / 63}$ & -- & Cut up & 32 & None & Destructively evaluated. \\
\hline 168 & $\underset{w / 0 \text { U }}{\mathrm{Zr}-2,11.1}$ & $\begin{array}{l}\text { Single } \\
\text { HWCTR } \\
\text { Driver }\end{array}$ & -- & $\stackrel{\text { NM }}{11 / 27 / 63}$ & -- & Cut up & 32 & None & Destructively evaluated. \\
\hline 169 & $\begin{array}{l}\mathrm{zr}-2,11.1 \\
\mathrm{w} / \mathrm{O} \cup\end{array}$ & $\begin{array}{l}\text { Single } \\
\text { HWCTR } \\
\text { Drtver }\end{array}$ & -- & $\stackrel{\mathrm{NM}}{1 / 7 / 64}$ & -- & Cut up & 32 & None & Destructively evaluated. \\
\hline $1-1$ & $\mathrm{Th}-1.6 \mathrm{w} / \mathrm{OU}$ & $\begin{array}{l}\text { Th metal } \\
\text { fuel tube }\end{array}$ & -- & $\underset{4 / 2 / 64}{\operatorname{SRL}}$ & -- & Cut up & 33 & None & Destructively evaluated. \\
\hline $1-2$ & $\mathrm{Th}-1.6 \mathrm{w} / \mathrm{o} \mathrm{v}$ & $\begin{array}{l}\text { Th metal } \\
\text { fuel tube }\end{array}$ & -- & $\begin{array}{l}\text { SRL } \\
4 / 2 / 64\end{array}$ & -- & Cut up & 33 & None & Destructively evaluated. \\
\hline $1-3$ & $T h-1.6 \mathrm{w} / \mathrm{o} \mathrm{U}$ & $\begin{array}{l}\text { Th metal } \\
\text { fuel tube }\end{array}$ & $\frac{\text { TMT-1 }}{(16)}$ & $\underset{4 / 2 / 64}{\text { SRI }}$ & -- & $\begin{array}{r}\text { Stored } \\
\text { SRL }\end{array}$ & 33 & None & Used for long term flow tests at SRL. \\
\hline$\infty$ & $\mathrm{Th}-1.6 \mathrm{w} / \mathrm{o} \mathrm{U}$ & $\begin{array}{l}\text { Th metal } \\
\text { fuel tube }\end{array}$ & - & $\begin{array}{c}\text { SRL } \\
7 / 9 / 64\end{array}$ & -- & $\begin{array}{c}\text { Stored } \\
\text { SRL }\end{array}$ & 33 & None & Th1s tube was not evaluated. \\
\hline $2-1$ & $\mathrm{Th}-1.5 \mathrm{w} / 0 \mathrm{U}$ & $\begin{array}{l}\text { Th metal } \\
\text { fuel tube }\end{array}$ & TMT-1-2 & $\begin{array}{l}\text { SRL } \\
7 / 9 / 64\end{array}$ & -- & $\begin{array}{c}\text { Irradiated } \\
\text { HWCTR }\end{array}$ & 33 & July 164 & $\mathrm{U}$ enriched to $93 \% 235 \mathrm{v}$ \\
\hline $2-2$ & $\operatorname{Th}-1.5 \mathrm{w} / \mathrm{O} \mathrm{U}$ & $\begin{array}{l}\text { Th metal } \\
\text { fuel tube }\end{array}$ & -- & $\begin{array}{c}\text { SRL } \\
7 / 9 / 64\end{array}$ & -- & $\begin{array}{c}\text { Stored } \\
\text { SRL }\end{array}$ & 33 & None & $\begin{array}{l}\text { U enriched to } 93 \% 235 \mathrm{U} \text {. Not suitable for Irradiation } \\
\text { because of ring markings on the inner surface at each } \\
\text { end of the tube. }\end{array}$ \\
\hline $2-3$ & $T h-1.5 \mathrm{w} / 0 \mathrm{U}$ & $\begin{array}{l}\text { Th metal } \\
\text { fuel tube }\end{array}$ & TMT-1-3 & $\begin{array}{c}\text { SRL } \\
7 / 9 / 64\end{array}$ & -- & $\begin{array}{l}\text { Irrad1ated } \\
\text { HWCTR }\end{array}$ & 33 & July 164 & $\mathrm{U}$ enriched to $93 \% 235 \mathrm{v}$ \\
\hline TWOTs & Unalloyed U & $\begin{array}{l}\text { Thin wall } \\
\text { outer tubes }\end{array}$ & $\begin{array}{l}\text { TWNT } \\
\text { (17) }\end{array}$ & $\underset{1962}{N M}$ & -- & $\begin{array}{l}8 \text { tubes 1r- } \\
\text { radiated } \\
\text { HWCTR. Re- } \\
\text { maining } 7 \\
\text { stored } \\
\text { SRL }\end{array}$ & 34 & $\begin{array}{l}\text { All tubes } \\
\text { auto- } \\
\text { claved }\end{array}$ & $\begin{array}{l}15 \text { of these tubes were fabricated as part of a nested } \\
\text { pairs of tubes for irradiation in HWCTR. } 7 \text { were 1rradi. } \\
\text { ated as nested pairs of outers and 1nners with spiral } \\
\text { ribbon spacers. Two of the assemblies failed. One was } \\
\text { irradiated individually as HWCTR Assy. No. TWO-1-2. }\end{array}$ \\
\hline TWITs & Unalloyed U & $\begin{array}{l}\text { Thin wall } \\
\text { Inner tubes }\end{array}$ & TWNT & $\begin{array}{l}N M \\
1962\end{array}$ & -- & $\begin{array}{l}7 \text { tubes } 1 \text { r- } \\
\text { rad1ated } \\
\text { HWCTR. Re- } \\
\text { ma1n1ng } 8 \\
\text { stored } \\
\text { SRL. }\end{array}$ & 35 & $\begin{array}{l}\text { All tubes } \\
\text { auto- } \\
\text { claved }\end{array}$ & Same as for TWOTs, 15 tubes fabricated. \\
\hline
\end{tabular}

(26) HWCTR number, TMT - Thorlum metal tube.

(17) HWCTR number, TWNT - Thin wall nested tubes. 


\section{Summary of Nuclear Metals Power Program Fuel Tubes (Continued)}

\begin{tabular}{|c|c|c|c|c|c|c|c|c|c|}
\hline $\begin{array}{l}\mathrm{MM} \\
\text { Mube } \\
\mathrm{Mbr}\end{array}$ & Composition & Description & $\begin{array}{l}\text { Irrad. } \\
\text { Assy: } \\
\text { Number }\end{array}$ & $\begin{array}{l}\text { Extruder } \\
\text { and Date }\end{array}$ & $\begin{array}{l}\mathrm{Mr} \\
\text { Extru. } \\
\text { Number }\end{array}$ & $\begin{array}{l}\text { Status } \\
\text { and } \\
\text { Location } \\
\end{array}$ & $\begin{array}{l}\text { Rer. } \\
\text { Info. }\end{array}$ & $\begin{array}{l}\text { Autoclave } \\
\text { Test } \\
\text { Completion }\end{array}$ & Comments \\
\hline $\begin{array}{c}M-1 \\
M-2 \\
\text { Drivers }\end{array}$ & $\mathrm{zr}-9.3 \times / 0 \mathrm{U}$ & $\begin{array}{l}\text { HWCTR } \\
\text { Drfver } \\
\text { Thubes }\end{array}$ & $\frac{M-1}{M-2} \approx$ & $\begin{array}{r}\mathrm{ABC} \\
1960\end{array}$ & -- & 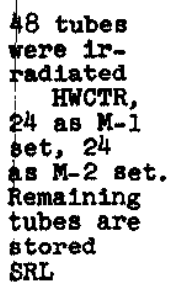 & 36 & $\begin{array}{l}13 \text { of the } \\
56 \text { tubes } \\
\text { tested }\end{array}$ & $\begin{array}{l}\text { U enriched to } 93 \% 235 \mathrm{U} \text {. } 56 \text { tubes were fabricated; } \\
53 \text { suitable for irradiation, and } 3 \text { rejected for minor } \\
\text { defects. }\end{array}$ \\
\hline $\begin{array}{l}x-3 \\
\text { Drivers }\end{array}$ & $\stackrel{2 r-2,1}{11.1} \times / 0 \quad 0$ & $\begin{array}{l}\text { BwCIR } \\
\text { Single } \\
\text { Trube } \\
\text { Driver }\end{array}$ & $M-3$ & $\begin{array}{c}\text { NM } \\
1964\end{array}$ & -- & $\begin{array}{l}\text { Stored } \\
\text { SRL }\end{array}$ & 37 & $\begin{array}{l}8 \text { of the } \\
28 \text { tubes } \\
\text { tested }\end{array}$ & $\begin{array}{l}\text { J enriched to } 93 \% 235 \mathrm{~J} \text {. } 28 \text { tubes were fabricated; } \\
24 \text { sultable for Irradiation. HWCTR operation term1- } \\
\text { nated prior to use of this driver set. }\end{array}$ \\
\hline
\end{tabular}


1. NM Tube Nos. 1-3

Ref: Progress Reports NMI-4355, 4356.

These three tubes were the first group of full-scale extmusions of Z1rcaloy-2 clad U-2 w/o $\mathrm{Zr}$ fuel. Extmusion was performed at $663^{\circ} \mathrm{C}$ with tube No. I not being extmuded. The remaining two tubes, Nos. 2 and 3 , were destructively examined following their extmusion. No detalied data were published.

2. NM Tube Nos. 4-9

Ref: (1) Progress Reports NMI-4350, 4B56, 4357.

(2) Letter, $3 / 25 / 58$, J. L. Klein, N.M. , to P. H. Permar, SRL, "SRI and NRU Flow Test tubes (5 and 8$)$ ".

Of this group of 6 tubes, 2 were shipped to SRL for flow tests. The remaining 4 tubes were cut up for destructive evaluation and other uses. Data for tubes 5 and 8 are presented below.

A. Description

Thick wall tubes, $2.060 "$ oD X $0.297^{\prime \prime}$ wall (nominal).

No. 5 - SRL flow test tube (Drawing ST-MDX3-1552)

No. 8 - NRU " " " (Drawing ST-MDX4-1598)

B. Composition

Core: U-2 w/o $\mathrm{Zr}$ alloy.

Cladding: Zircaloy-2 ( $15 \mathrm{mils}$ nominal thickness).

End seals: Integral-type Zircaloy-2.

C. Dimensions

Taken before final bright etching. Approximately 2 mils removed from each surface during bright etching; therefore, I.D. should be increased by $\sim 0.004^{\prime \prime}$ and $0 . D$. decreased by $\sim 0.004^{\prime \prime}$.

Over uniform core oD

\begin{tabular}{c}
$\begin{array}{c}\text { Tube 5 } \\
\text { (1nches) }\end{array}$ \\
\hline 2.060 \\
1.447 \\
2.079 \\
1.464 \\
132
\end{tabular}

Tube 8

Over uniform core OD

Over end seals, OD

overall length

ID

Core, tip-to-tip

$125-7 / 8$

(inches)

2.056

1.447

2.079

1.467

$132 / 8-7 / 8$ 
D. Extrusion Conditions

(Done at Revere Copper \& Brass Co., Detro1t, M1chigan)

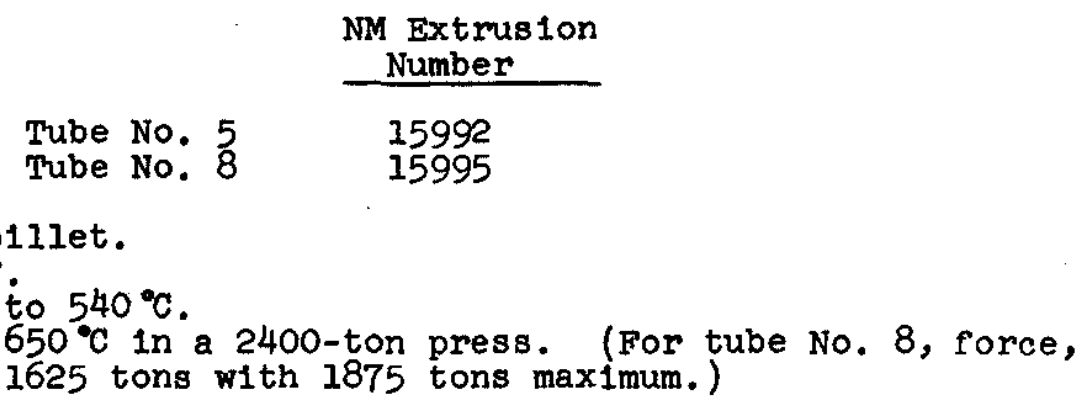

E. Heat Treatment - none.

F. Autoclave Test - none.

G. Remarks

Both tubes were used for flow tests and HWCTR zero power tests at SRL.

H. Current Conditions

Tubes 5 and 8 are stored at SRL.

These tubes are not suitable for 1rradiation.

3. NM Tube Nos. 10-16

Ref: (1) Progress Reports NMI-4357, 4358, 4359, 4361, 4362, 4366.

(2) NMI-4360, 4/19/58, J. I. Klein, "Evaluation of Three Zircaloy-clad U-2 w/o Zr Fuel Tubes (11, 14 \& 15)".

Four of the 7 tubes were sent to SRL, 2 ( $1 I$ and 15) were 1rradiated at SRP, 1 (14) used for flow tests, and 1 (16) used for HWCTR zero power tests. The remaining three tubes were cut up for evaluation and other uses. Data for tubes 11, 14, 15 and 16 are presented below.

A. Description

Thick-wall tube, $2.060^{n}$ oD X $0.297^{n}$ wall (nominal)

B. Composition

Core: U-2 w/o Zr alloy.

Cladding: Zircaloy-2 (15 mils).

End seals: Integral-type Z1rcaloy-2 


\section{Dimensions}

Taken before final bright-etching (ID should be increased $0.002^{\prime \prime}$ and OD decreased by $\left.0.002^{\prime \prime}\right)$. Tube 16 after etching and before heat treatment.

Tube 11 (inches)

Over uniform core, OD

Over end seals, OD

Overall length

Core, tip-to-tip

2.065
1.470
2.073
1.476
$132-1 / 8$
$117-3 / 8$

Tube 14 (Inches)

Tube 15 (inches)

Tube 16

2.064

1. 468

2.077

1.475

$132-1 / 4$

$118-3 / 8$

$\begin{array}{ll}2.064 & 2.055 \\ 1.472 & 1.456 \\ 2.072 & 2.076 \\ 1.477 & 1.474 \\ 132-1 / 4 & 132-1 / 4 \\ 119-5 / 8 & 119-9 / 16\end{array}$

D. Extrusion Conditions

(Done at Revere Copper \& Grass Co., Detroit, Michigan)

Tube 11 Tube 14 Tube 15 Tube 16

NM extrusion number

Tool temperature

$\begin{array}{cccc}16241 & 16244 & 16431 & 16432\end{array}$

Billet temperature $\left({ }^{\circ} \mathrm{C}\right.$ )

Extrusion force, running (tons)

Extrusion force, max. (tons)

Ram speed (inches/min)

540

650

1800

540

2100

1850

650

650

25

2050

350

1900

Billets, copper-clad

Copper cutoff

E. Heat Treatment

No. 11 held at $800^{\circ} \mathrm{C}$ for $\frac{1}{2}$ hour supported by Mo pads; rapid cool.

No. 14 held at $880^{\circ} \mathrm{C}$ for 7 hours wrapped in Ta foil; rapid cool. Tantalum stuck to zircaloy cladding causing indentations and pits.

No. 15 held at $880^{\circ} \mathrm{C}$ for 7 hours supported by Mo pads; rapid cool.

No. 16 held at $890^{\circ} \mathrm{C}$ for 7 hours supported by type $304 \mathrm{stain-}$ less steel; rapid cool.

F. Autoclave Test

No. 11 tested for 24 hours at $345^{\circ} \mathrm{C}$ in water. Black tarnish film with a few white spots near end.

No. 14 tested for 24 hours at $345^{\circ} \mathrm{C}$ in water. Write spots running length of cladding attributed to faulty rinsing of etching solution.

No. 15 tested for 24 hours at $345^{\circ} \mathrm{C}$ in water. General black tarnish film with some white spots. 


\section{G. Remarks}

None.

H. Current Conditions

Tubes 14 and 16 are stored at SRL. No. 14 may be suitable for irradiation; No. 16 is not.

4. NM Tube Nos, 17-21

Ref: (1) Progress Reports NMI-4359, 4361, 4366.

(2) NMI-4365, 7/24/58, J. L. Klein, "Evaluation of Z1rcaloy-clad U-2 w/o Zr Alloy Tube No. 19, Extrusion No. $16819^{\prime \prime}$.

Three of the f1ve tubes were sent to SRL, two (18 and 20) were used for HWCTR zero power tests, and one (19) was irradiated at SRP. Tube No. 20 was used 28 a demonstration tube and for trial handling runs in Canada before belng sent to SRL. The remaining two tubes were cut up for evaluation and other uses. Data for tubes 18,19 and 20 are presented below.

A. Description

Th1ck-wall tube, 2.060" oD X $0.297^{\prime \prime}$ wall (nominal).

B. Composition

Core: U-2 w/o $\mathrm{Zr}$ alloy

Cladding: Zircaloy-2 (is mils)

End seals: Integral-type Zircaloy-2

C. D1mensions

Taken before final bright-etching (ID should be increased $\sim 0.002^{\prime \prime}$ and OD decreased by $\left.\sim 0.002^{\prime \prime}\right)$. Tube 18 after etching and before heat treatment.

\begin{tabular}{|c|c|c|c|}
\hline & $\begin{array}{l}\text { Tube } 18 \\
\text { (inches) }\end{array}$ & $\begin{array}{l}\text { Tube } 19 \\
\text { (inches) }\end{array}$ & $\begin{array}{l}\text { Tube } 20 \\
\text { (Inches) }\end{array}$ \\
\hline $\begin{array}{l}\text { Over uniform core, OD } \\
\text { Over end seals, OD } \\
\text { Overall length ID }\end{array}$ & $\begin{array}{c}2.054 \\
1.456 \\
2.070 \\
1.472 \\
132\end{array}$ & $\begin{array}{c}2.063 \\
1.468 \\
2.070 \\
1.476 \\
132 \\
110\end{array}$ & $\begin{array}{c}2.058 \\
1.467 \\
2.066 \\
1.472 \\
132\end{array}$ \\
\hline
\end{tabular}


D. Extrusion Conditions

(Done at Revere Copper \& Brass Co., Detrolt, Michigan)

Thube 18 Tube 19 Tube 20

NM extmusion number

Tool temperature $\left({ }^{\circ} \mathrm{C}\right)$

Billet heat temp. $\left({ }^{\circ} \mathrm{C}\right)$

Extrusion force, munnin

Ram speed (inches/min)

Extrusion constant, munning (tsi)

Extrusion ratio $\begin{array}{lll}16818 & 16819 & 16820\end{array}$

Billets, copper-clad

$\begin{array}{lll}- & 540 & - \\ - & 650\end{array}$

Copper cutoff

E. Heat Treatment

No. 19 held at $890^{\circ} \mathrm{C}$ for 7 hours supported by a ground, type 304 stainless steel angle; rapid cool.

No. 20 held at $880^{\circ} \mathrm{C}$ for 7 hours supported by Mo pads followed by water quenching.

F. Autoclave Test

No. 19 tested for 24 hours at $345^{\circ} \mathrm{C}$ in water followed by 24 hours at $400^{\circ} \mathrm{C}$ in $1500 \mathrm{psi}$ steam. Hard, lustrous, black oxide film formed.

No. 20 tested for 24 hours at $345^{\circ} \mathrm{C}$ in water, followed by 24 hours at $400^{\circ} \mathrm{C}$ in 1500 psi steam. Satisfactory corrosion resistance shown.

G. Remarks

None.

H. Current Condition

Nos. 18 and 20 are stored at SRL. Ne1ther 1s considered suitable for irradiation. 
5. NM Tube Nos. 22-32

Ref: (1) Progress Reports NMI-4361, 4363, 4366, 4381, 4382, 4383.

(2) NMI-4374, 2/13/59, D.F.Kaufman and R.G.Jenkins, "Evaluation of Zircaloy-Clad U-2 w/o Zr Alloy Tube No. 22".

(3) NMI-4375, 2/19/59, D.F.Kaufman and R.G.Jenkins, "Evaluation of Zircaloy-Clad U-2 w/o $\operatorname{~rr}$ Alloy Tube No. $23 "$ ".

(4) NMI-4376, 2/26/59, D.F.Kaufman and R.G.Jenkins, "Evaluation of Zircaloy-Clad U-2 w/o Zr Alloy Tube No. 25".

(5) NMI-4372, 1/9/59, D.F.Kaufman and R.G.Jenkins, "Evaluation of Zircaloy-Clad U-2 w/o $\mathrm{Zr}$ Alloy Tube No. 26".

(6) NMI-4377, 4/13/59, D.F.Kaufman and R.G.Jenkins, "Evaluation of Zircaloy-Clad U-2 w/o $\mathrm{Zr}$ Alloy Tube No. 28."

(7) NMI-4377--Supplement, 7/29/60, D.F.Kaufman, R.G.Jenkins, W.B.Tuffin, "Spheroldization Heat Treatment and Re-evaluation of Zircaloy-Clad U-2 w/o $\mathrm{Zr}$ Alloy Tube No. 28".

(8) NMI-4370, 12/16/58, D.F.Kaufman and R.G.Jenkins, "Complete Set of Tables for Power Tube No. 29".

(9) NMI-4371 (Rev.), 12/23/58, D.F.Kaufman and R.G.Jenkins, "Evaluation of Zircaloy-Clad U-2 w/o Zr Alloy Tube No. 30 ".

(10) NMI-4373, 1/12/59, D.F.Kaufman and R.G.Jenkins, "Evaluation of Zircaloy-Clad U-2 w/o Zr Alloy Tube No. 31".

(11) NMI-4378, 4/13/59, D.F.Kaufman and R.G.Jenk1ns, "Evaluation of Zircaloy-Clad $U-2$ w/o Zr Alloy Tube No. $32 "$ ".

To demonstrate a prototype semi-production operation for manufacture of co-extruded, long, tubular fuel elements, a total of 11 tubes was scheduled--an initial mun of 2 tubes followed by a group of 9 tubes. This group of tubes was the first to be scheduled at American Brass Co. (ABC). These and all subsequent thick-walled tubes were extruded at $A B C$ primarily because of close location.

The first 2 tubes (22 and 23) were extruded on schedule; only 4 of the remaining 9 were on schedule; 2 (26 and 28) extruded successfully and 2 ( 24 and 27 ) unsuccessfully. All of the remaining 5 prototype tubes $(25,29-32)$ were later successfully extruded after modification of billet handiing procedures at $\mathrm{ABC}$.

of the 11 tubes in this program, $4(22,28,29,30)$ were irradiated at SRP; $3(26,31,32)$ were used for HWCTR zero power tests and are stored at SRL; and the remaining $4(23,24,25,27)$ were cut up for evaluation and other uses. Data for the irradiated tubes and those stored at SRL are presented below. 
A. Description

Thick-wall tubes, $2.060^{\prime \prime}$ OD X $0.297^{\prime \prime}$ wall (nominal).

Draw1ng STD-MDX-4-1598, Rev. 3 .

B. Composition

Core: U-2 w/o $\mathrm{Zr}$ alloy.

Cladding: Zircaloy-2 ( $15 \mathrm{mlls}$ ).

End seals: Integral-type Z1rcaloy-2.

C. Dimensions

Taken before final bright-etching; ID should be increased $\sim 0.002^{\prime \prime}$ and OD decreased $\sim 0.002^{\circ}$.

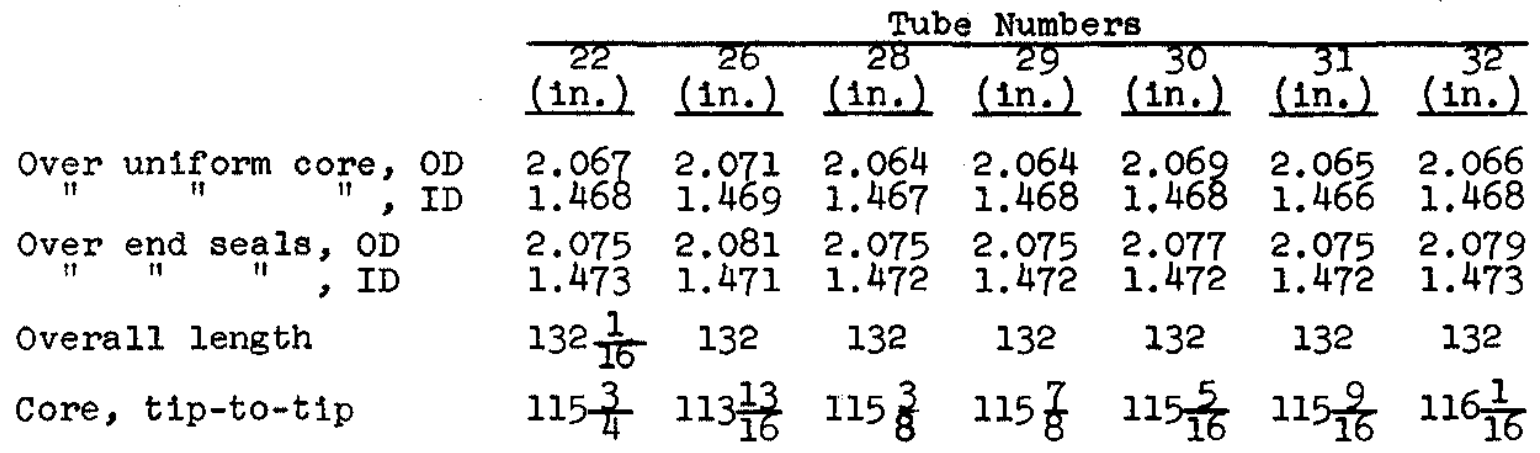

D. Extmusion Conditions

(Done at American Brass Co.)

\begin{tabular}{|c|c|c|c|c|c|c|c|}
\hline & \multicolumn{7}{|c|}{ Tube Numbers } \\
\hline & 22 & 26 & 28 & 29 & 30 & $3 I$ & 32 \\
\hline NM extrusion number & 18393 & 18386 & 18388 & 18389 & 18390 & 18391 & 18392 \\
\hline Tool temperature $\left({ }^{\circ} \mathrm{C}\right)$ & $\begin{array}{l}430- \\
480(1)\end{array}$ & $\begin{array}{l}430- \\
480\end{array}$ & $\begin{array}{l}430- \\
480\end{array}$ & $\begin{array}{l}430- \\
480\end{array}$ & $\begin{array}{l}430- \\
480\end{array}$ & $\begin{array}{l}430- \\
480\end{array}$ & $\begin{array}{l}430- \\
480\end{array}$ \\
\hline Billet temp. $\left({ }^{\circ} \mathrm{C}\right)$ & 645 & 665 & 660 & 650 & 650 & 650 & 650 \\
\hline $\begin{array}{l}\text { Extmusion force, run- } \\
\text { ning (tons) }\end{array}$ & 1630 & 1630 & 1670 & 1600 & 1600 & 1600 & 1600 \\
\hline $\begin{array}{l}\text { Extmusion force, maxi- } \\
\text { mum (tons) }\end{array}$ & 1800 & 1730 & 1800 & 1770 & 1800 & 1880 & 1880 \\
\hline Ram speed (Inches/min) & 25 & 25 & 25 & 25 & 25 & 25 & 25 \\
\hline $\begin{array}{l}\text { Extmusion constant, } \\
\text { munning (ts1) }\end{array}$ & 17.1 & 17.1 & 17.5 & 16.8 & 16.8 & 16.8 & 16.8 \\
\hline $\begin{array}{l}\text { Extrusion ratio } \\
\text { Billets, copper-clad } \\
\text { Copper cutoff }\end{array}$ & 18.3 & 18.3 & 18.3 & 18.3 & 18.3 & 18.3 & 18.3 \\
\hline
\end{tabular}

(1) Liner at $430^{\circ} \mathrm{C}$; die and mandrel at $480^{\circ} \mathrm{C}$. 
E. Heat Treatment

Tubes $22,26,29,30$ and 32 were held at $890^{\circ} \mathrm{C}$ for 7 hours supported by a graphite strip-Iined stainless steel angle; rapid cool (water quenching of evacuated tube container).

No. 28 held at $890^{\circ} \mathrm{C}$ for 9 hours; rapid cool. Following autoclaving, this tube recelved a spheroldization heat treatment at New England Metaliurgical Corp. The tube was held in the range $670-695^{\circ} \mathrm{C}$ for 72 hours, and rapid cooled by quenching the evacuated container in a $4 \%$ caustic solution.

No. 31 held at $890^{\circ} \mathrm{C}$ for 9 hours and rapld cooled.

F. Autoclave Test

All 7 tubes were tested for 24 hours at $345^{\circ} \mathrm{C}$ in water followed by 24 hours at $400^{\circ} \mathrm{C}$ in $1500 \mathrm{ps} 1$ steam. All tubes had a continuous, hard, Iustrous, black oxide film assoclated with Zircaloy having good corrosion resistance to high temperature water.

G. Remarks

None.

\section{H. Current Condition}

Nos. 26, 31 and 32 are stored at SRL. All three tubes are considered sultable for irradiation.

6. NM Tube Nos. 33-42

Ref: (1) Progress Reports NMI-4369, 4381, 4382, 4383, 4384, $4385,4386,4392,4393$.

(2) NMI-4389, 6/2/59, D.F.Kaufman and W.J.R1chmond, "Evaluation of Zircaloy-Clad U-2 w/o $\mathrm{Zr}$ Alloy Tube No. 36".

(3) NMI-4387, 6/10/59, D.F.Kaufman and W.J.R1chmond, "EvaIuation of S1X Zircaloy-Clad U-2 w/o $\mathrm{Zr}$ Alloy Tubes Made for Irradiation in the VBWR Test Loop $(37-42)^{\prime \prime}$.

(4) NMI-4380, 1/7/59, W.J.Richmond and D.F.Kaufman, "Evaluation of Zircaloy-Clad U-2 w/o Zr Alloy Tube No. $34 "$. 
A group of ten fuel tubes was fabricated as part of a program to provide an irradiation candidate for testing in the General Electric Bolling Water Test Reactor (VBWR), Vallecitos, California.

The first four tubes (33-36) used natural uranium and served as prototypes. Of these, one (34) was irradiated at SRP and one (35) was used for SRL flow tests.

The second group of tubes $(37-42)$ used uranium enriched to $3.06 \% 235 \mathrm{U}$. No. 42 was irradiated at VBWR; No. 38 was destructively evaluated. The remaining four tubes, three of which are suitable for irradiation, are stored at SRL.

Data for one of the natural uranium tubes (34) and five of the enriched tubes are presented below.

A. Description

Thick-wall tubes designed for use in the VBWR, 2.060" OD X $0.297^{\prime \prime}$ wall (nominal). Drawing ST-MDX-2253.

B. Composition

Core: U-2 w/o Zr alloy.

Tubes $37-42$, U enriched to $3.06 \% 235 \mathrm{U}$.

Cladding: Zircaloy-2 ( $15 \mathrm{mils}$ ).

End seals: Integral-type Zircaloy-2.

C. Dimensions

Taken before final bright-etching. (ID should be increased $\sim 0.003$ and OD decreased by $\sim 0.003^{\prime \prime}$.

Tube Numbers

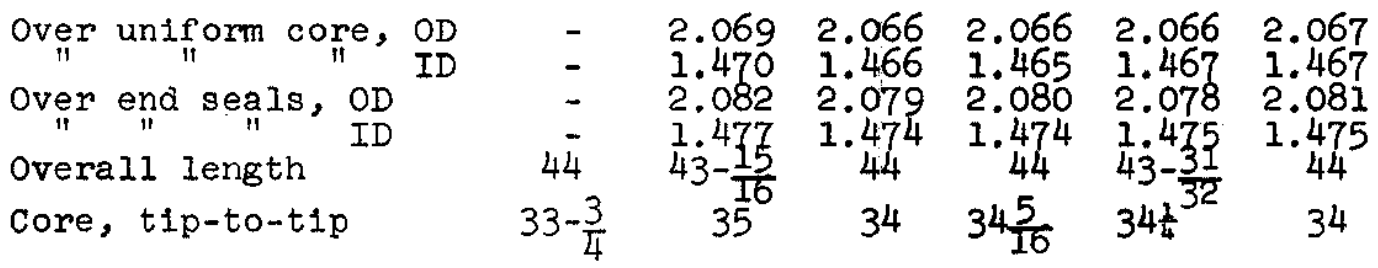




\section{Extrusion Conditions}

(Done at American Brass Co.)

NM extrusion number

Tool temperature $\left({ }^{\circ} \mathrm{C}\right)$

Billet temperature $\left({ }^{\circ} \mathrm{C}\right)$

Extrusion force, running

(tons)

Extrusion force, maximum (tons)

Ram speed (Inches/min)

Extrusion constant, munning (ts1)

Extrusion ratio

Billets, copper-clad

Copper cutoff
Tube Numbers

\begin{tabular}{|c|c|c|c|c|c|}
\hline 34 & 37 & 39 & 40 & $4 I$ & 42 \\
\hline 19658 & 19889 & 19897 & 19892 & 19893 & 19894 \\
\hline${ }_{430-}^{430}$ & $\begin{array}{r}430- \\
480\end{array}$ & $\begin{array}{c}430-! \\
480\end{array}$ & $\begin{array}{r}430- \\
480\end{array}$ & $\begin{array}{r}430- \\
480\end{array}$ & $\begin{array}{r}430- \\
480\end{array}$ \\
\hline 655 & 650 & 645 & 650 & 650 & 650 \\
\hline 1550 & 1770 & 1530 & 1570 & 1520 & 1500 \\
\hline 1760 & 1900 & 1690 & 1640 & 1670 & 1670 \\
\hline 25 & 25 & 25 & 25 & 25 & 25 \\
\hline 16.4 & 17.0 & 16.0 & 16.4 & 15.9 & 15.7 \\
\hline 18.3 & 18.3 & 18.3 & 18.3 & 18.3 & 18.3 \\
\hline
\end{tabular}

E. Heat Treatment

All tubes were held at $890^{\circ} \mathrm{C}$ for 7 hours and rapld-cooled.

F. Autoclave Test

Nos. $34,37,39-42$ were tested for 24 hours at $345^{\circ} \mathrm{C}$ in water followed by 24 hours at $400^{\circ} \mathrm{C}$ in 1500 psi steam. There was no evidence of bond-line corrosion at the tube ends after autoclaving. Only tube 39 had white oxide after autoclaving;

all others showed lustrous black oxide.

G. Remarks

No. 34 failed during irradiation at SRP.

No. 41 was Irradiated to $1200 \mathrm{MWD} / \mathrm{T}$ in the VBWR.

H. Current Condition

Tube Nos. 35, 37, 39, 40, 41 (Irradiated in VBWR) and 42 are stored at SRL. Tubes 35 and 37 are considered unsuitable for irradiation.

Tube Nos 33 and 36 are stored at NM.

(1) Liner at $430^{\circ} \mathrm{C}$; die and mandrel at $480^{\circ} \mathrm{C}$. 
7. NM Tube Nos. $44-49$

Ref: Progress Reports NMI-4383, 4384, 4385, 4386, 4392, 4393, 4394.

A group of $s i x$ prototype tubes was fabricated to develop a process for production of driver tubes for HWCTR. All but one (44, used for flow tests) were destructively evaluated. Data for tube 44 are presented below.

A. Description

Prototype HWCTR driver tubes, 2.300" OD X $0.167^{\prime \prime}$ wall

(nominal).

B. Composition

Core: Zr-9.3 w/o U alloy.

Cladding: Zircaloy-2 (15 mils).

End seals: Integral-type Z1rcaloy-2.

C. Dimensions

No. 44 was cold-drawn from its as-extmuded size of $2.367^{\prime \prime}$ OD

$\mathrm{X} 1.976^{\prime \prime}$ ID to $2.308^{\prime \prime}$ OD $\mathrm{X} 1.964^{\prime \prime}$ ID in two passes.

Overall length: 118 ".

Core length, tip-to-tip: 99-15/16"

Bottom end seal length (front as-extmuded): 2 ".

D. Extrusion Data

Not reported.

E. Heat Treatment

None.

F. Autoclave Test

None.

G. Remarks

Based on the $11.2 \%$ cold-drawing of tube 44 to s1ze, as determined from length measurements, cold-drawing has been eliminated from the process, with ail future tubes extruded to size.

H. Current Condition

Tube 44 is stored at SRL. 
8. NM Tube Nos. 50-55

Ref: (1) Progress Reports NMI-4392, 4393, 4394, 4395, 4396, $4397,4398,7221$.

(2) NMI-4394, 11/19/59, W.I.Larson, "Evaluation of Zircaloy-Clad Unalioyed Uranium Tube No. 53".

To broaden the base of the power tube irradiation program at SRP, NM fabricated a group of 5 dingot-base unalloyed urantum tubes, similar in dimensions to the previous $U-2 \mathrm{w} / 0 \mathrm{Zr}$ cored power tubes. One tube, No. 53, was Irradiated at SRP. Data for No. 53 are presented below.

A. Description

Unalloyed $U$ thick-wall tubes, 2.060" OD X $0.297^{\prime \prime}$ wall (nomina1).

B. Composition

Core: Unalloyed U.

Cladding: Z1rcaloy-2 ( $30 \mathrm{mils}$ ).

End seals: Integral-type Zircaloy-2.

C. Dimensions

Taken before final bright-etching; (ID should be increased $\sim 0.003^{\prime \prime}$ and OD decreased $0.003^{\circ}$.)

$\begin{array}{lc} & \begin{array}{c}\text { Tube 53 } \\ \text { (inches) }\end{array} \\ \text { Over uniform core, OD } & 2.057 \\ \text { "Ner end seals, OD } & 1.469 \\ \text { "ID } & 2.070 \\ \text { Overall Iength" ID } & 1.475 \\ \text { Core, tip-to-tip } & 132 \\ & 108 \frac{1}{4}\end{array}$

D. Extrusion Conditions

(Done at American Brass Co.)

NM extrusion number

Tool temperature $\left({ }^{\circ} \mathrm{C}\right)$

Billet temperature $\left({ }^{\circ} \mathrm{C}\right)$

Extrusion force, running (tons)

Extrusion constant, running (ts1)

Ram speed (inches/min)

Extrusion ratio

Billets, copper-clad

Copper cutoff

(1) Liner at $430^{\circ} \mathrm{C}$; die and mandrel at $480^{\circ} \mathrm{C}$. 


\section{E. Heat Treatment}

Heat treatment was done at the Hick Corporation, Hyde Park, Mass., using a Holden Type-70I vertical salt bath furnace. The tube, in 1ts copper facket, was immersed in a $730^{\circ} \mathrm{C}$ salt bath for 10 minutes, alr-cooled to $200^{\circ} \mathrm{C}$, then water-quenched.

F. Autoclave Test

No. 53 was tested for 4 hours at $345^{\circ} \mathrm{C}$ in water followed by $6^{2}$ hours at $400^{\circ} \mathrm{C}$ in $1500 \mathrm{ps} 1$ steam. A continuous, hard, lustrous, black oxide film resulted. The insurance welds at the tube ends had a white oxide layer.

G. Remariks

Tube 55 was extruded from a tandem billet containing two short cores, one with high carbon and one with low carbon, for characterization of the full-length tubes.

Because of inferior bond quality at the integral end seals of No. 53, insurance welds were made at both tube ends at the end-seal-cladding interfaces.

No. 5$]$ was used for HWCTR zero power tests.

\section{H. Current Condition}

Tube 5.1 is stored at SRL, and tubes 54 and 55-A are stored at NM. None of these tubes are sultable for irradiation.

9. NM Tube Nos. 56-60

Ref. (1) Progress Reports NMI-4395, 4396, 4397.

(2) NMI-4379, 4/26/60, A.M.Huntress and D.F.Kaufman, "Evaluation of Three Enriched HWCTR Driver Tubes for Irradiation at SRP (58-60)"

A second group of five prototype HWCTR, two natural U $(56,57)$ and three enriched $(58-60)$, were fabricated to obtain enriched tubes for irradiation tests at SRP. This second group of prototypes differed from the first set (Item 7 of this Appendix) in their being extruded directly to size rather than 15\% oversize for subsequent cold-drawing to size. Two of these enriched tubes $(59,60)$ were irradiated at SRP with the third (58) used as a Nuclear Test Gage (NTG) standard. The two natural U tubes were used to evaluate core length. Data for the enriched tubes are presented below. 
A. Description

Prototype HWCTR driver tubes, $2.300^{\prime \prime}$ OD X $0.167^{\prime \prime}$ wall

(nominal).

Drawing STD-MDX4-2346.

B. Composition

Core: $\mathrm{Zr}-9.3 \mathrm{w} / \mathrm{O} \mathrm{U}$ alloy (Nos. 58-60, U enriched to $93 \% 235 \mathrm{U}$ ).

Cladding: Z1rcaloy-2 ( 15 mils).

End seals: Integral-type Z1rcaloy-2.

C. Dimensions

Taken before final bright-etching. (ID should be increased $0.003^{\prime \prime}$ and OD decreased by $0.003^{\circ}$.)

Tube Numbers

\begin{tabular}{|c|c|c|}
\hline $\begin{array}{c}58 \\
\text { (Inches) }\end{array}$ & $\begin{array}{c}59 \\
\text { (Inches) }\end{array}$ & $\begin{array}{c}60 \\
\text { (inches) }\end{array}$ \\
\hline $\begin{array}{l}2.302 \\
1.961\end{array}$ & $\begin{array}{l}2.300 \\
1.961\end{array}$ & $\begin{array}{l}2.298 \\
1.961\end{array}$ \\
\hline $\begin{array}{l}2.302 \\
1.960\end{array}$ & $\begin{array}{l}2.300 \\
1.960\end{array}$ & $\begin{array}{l}2.298 \\
1.960\end{array}$ \\
\hline 118 & 118 & 118 \\
\hline $112-3 / 16$ & $112-1 / 4$ & $112-3 /$ \\
\hline
\end{tabular}

D. Extrusion Conditions

(Done at American Brass Co.)

NM extrusion number

Tool temperature $\left({ }^{\circ} \mathrm{C}\right)$

Billet temperature $\left({ }^{\circ} \mathrm{C}\right)$

Extrusion force, running (tons)

Extrusion force, maximum (tons)

Extrusion constant, running (ts1)

Ram speed (inches/min)

Extrusion ratio

Tube Numbers

\begin{tabular}{|c|c|c|}
\hline 58 & 59 & 60 \\
\hline $\begin{array}{c}21336 \\
430-480(1) \\
690\end{array}$ & $\begin{array}{c}21337 \\
430-480 \\
690\end{array}$ & $\begin{array}{c}21338 \\
430-480 \\
690\end{array}$ \\
\hline 1250 & 1170 & 1210 \\
\hline 1330 & 1290 & 1330 \\
\hline 20.6 & 19.3 & 20.0 \\
\hline $\begin{array}{c}25 \\
17.1\end{array}$ & $\begin{array}{c}25 \\
17.1\end{array}$ & $\begin{array}{c}25 \\
17.1\end{array}$ \\
\hline
\end{tabular}

Billets, copper-clad

Copper cutoff

(1) Liner at $430^{\circ} \mathrm{C}$; die and mandrel at $480^{\circ} \mathrm{C}$. 
E. Heat Treatment

None.

F. Autoclave Test

The three enriched tubes were tested for 4 hours at $345^{\circ} \mathrm{C}$ in water followed by 4 hours at $400{ }^{\circ} \mathrm{C}$ in 1500 psi steam. A lustrous, black oxide resulted.

\section{Q. Remarks}

Tube 58 was not suitable for irradiation because of clad thinning in scored areas of 2 to 6 mils (minimum clad specification - 10 mils). Tube was utilized as a standard for the Nuclear Test Gage at SRP.

\section{H. Current Condition}

Tube 58 is stored at SRL; it is not sultable for irradiation.

10. NM Tube Nos. 61-63

Ref: Progress Reports NMI-4395, 4396, 4397, 7221, 7222, 7223.

To produce experimental power tubes with uranium alloys other than $U-2 \mathrm{w} / 0 \mathrm{Zr}$, a group of three tubes was fabricated. Two of the tubes were full-length, one with a core of U-1 w/o S1 (62) and the other with a core of U-1.5 w/o Mo (63). The third tube $(61 \mathrm{~A}, 61 \mathrm{~B})$ was a tandem extrusion containing a short core of each of the two compositions used for destructive evaluation to characterize the full-size tubes. Data for the full-length tubes used for HWCTR zero power test are presented below.

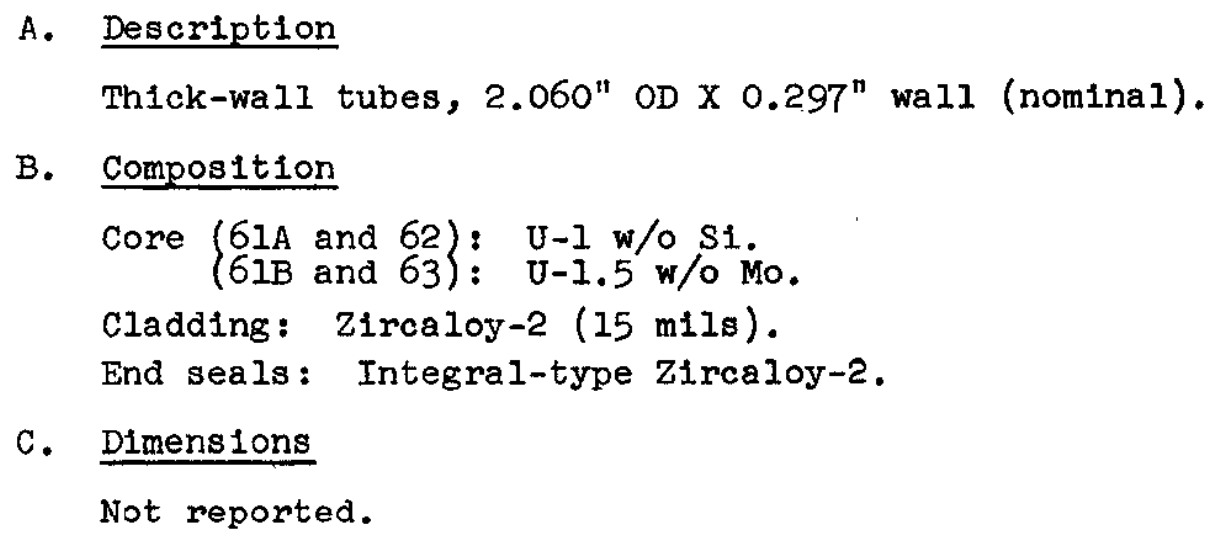


D. Extrusion Conditions

Not reported.

E. Heat Treatment

Tube 62 was beta-treated in a salt bath for 10 minutes at $730^{\circ} \mathrm{C}$ and air-cooled in its copper extrusion jacket.

Tube 63 recelved no heat treatment.

F. Autoclave Test

None.

G. Remarks

Several depressions up to $6 \frac{1}{2} \mathrm{mils}$ deep were observed on the OD over the untform core of Tube 62 . Clad thinning on the inner surface near the rear end seal was observed on Tube 63 . Neither tube was considered suitable for irradiation.

H. Current Condition

Tubes 62 and 63 are stored at SRL. They are not sultable for irradiation.

11. NM Tube Nos. 64-69

Ref: (1) Progress Reports NMI-4398, 7221, 7222, 7223, 7224.

(2) NMI-7200, 4/25/60, W.L.Iarson, "Evaluation of Z1rcaloyClad Unalloyed Uranlum Tubes Made For Irradiation in the VBWR Test Loop $(68,69) "$.

Th1s group of thick-wall tubes consisted of four short natural U prototypes (64-67) to investigate the effect of billet end shapes on clad thinning and two enriched $U$ tubes $(68,69)$ for Irradiation testing at the VBWR. The four prototype tubes were destructively evaluated. Although the two enriched tubes are suitable for irradiation, they were not used and are currently stored at SRL. Data for the enriched tubes are presented below.

A. Description

Th1ck-wall VBWR tubes, 2.060" OD X $0.297^{\prime \prime}$ wall (nominal). Drawing MDX4-2253.

B. Composition

Core: Unalloyed $U(68,69-U$ enriched to $3.10 \%$ 235U)

Cladding: Z1rcaloy-2 (30 $\mathrm{mlls}$ ).

End seals: Integral-type zircontum. 
C. Dimensions

Taken before final bright-etching. (ID should be increased $0.003^{\prime \prime}$ and $O D$ decreased by $0.003^{n}$.)

\begin{tabular}{|c|c|c|}
\hline & $\begin{array}{r}\text { Tube } 68 \\
\text { (Inches) }\end{array}$ & $\begin{array}{l}\text { Tube } 69 \\
\text { (inches) }\end{array}$ \\
\hline Over uniform core, OD & $\begin{array}{l}2.067 \\
1.468\end{array}$ & $\begin{array}{l}2.066 \\
1.467\end{array}$ \\
\hline Over end seals, OD & $\begin{array}{l}2.082 \\
1.472\end{array}$ & $\begin{array}{l}2.080 \\
1.469\end{array}$ \\
\hline $\begin{array}{l}\text { Overall length } \\
\text { Core, tip-to-tip }\end{array}$ & $\begin{array}{l}50 \\
39-5 / 8\end{array}$ & $\begin{array}{l}50 \\
40-1 / 2\end{array}$ \\
\hline
\end{tabular}

D. Extmusion Conditions

(Done at American Brass Co.)

NM extrusion number

Tool temperature $\left({ }^{\circ} \mathrm{C}\right)$

Billet temperature $\left({ }^{\circ} \mathrm{C}\right)$

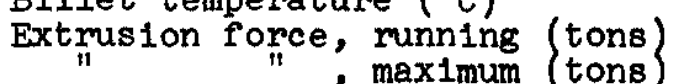

Extrusion constant, running (tsi)

Ram speed (inches/min)

Extrusion ratio

Billets copper-clad

Copper cutoff

\begin{tabular}{cc} 
Trube 68 & Thube 69 \\
\cline { 2 - 2 } & 27323 \\
$430-480(1)$ & 27324 \\
630 & $430-480$ \\
1230 & 630 \\
1660 & 1260 \\
12.9 & 1650 \\
25 & 13.2 \\
18.3 & 25 \\
& 18.3
\end{tabular}

E. Heat Treatment

Both enriched tubes were beta-treated in a salt bath for 10 minutes at $700^{\circ} \mathrm{C}$ and air-cooled in their copper extrusion jackets.

F. Autoclave Test

The two tubes were tested for 4 hours at $345^{\circ} \mathrm{C}$ in water followed by 4 hours at $400^{\circ} \mathrm{C}$ in 1500 ps 1 steam. Both tubes exhibited a continuous, hard, iustrous, black oxide film with no white oxide observed.

G. Remarks

Four different core end shape designs were extruded $(64,67)$ to obtain the design used for the two enriched tubes. The core end shape selected had six-degree compensated front and rear end shapes; single tooth in middle of front and rear end seal walis interlocking with core; also $1 / 8-1$ nch, $10^{\circ}$ chamfers on all four corners of core.

(1) Iiner at $430^{\circ} \mathrm{C}$; die and mandrel at $480^{\circ} \mathrm{C}$. 
Both enriched tubes had clad thinning on the oD near the end seals $\left(0.011^{\prime \prime}\right.$ versus a specified $\left.0.030 \pm 0.003^{\prime \prime}\right)$. However, both tubes were considered suitable for Irradiation testing.

\section{H. Current Condition}

Tubes 68 and 69 are stored at SRI and are considered suitable for irradiation.

12. NM Tube Nos. $70-71$

Ref: Progress Reports NMI-7224, 7225, 7226.

Two thick-wall tubes (2.060" OD X $0.297^{\prime \prime}$ wall) having different core compositions, U-1 w/O SI (70) and U-1.5 w/o Mo (71), were fabricated as prototypes for production of similar tubes for the VBWR. Both tubes were destructively evaluated.

Work on thlck-wall tubes was discontinued because of a design change from single thick-wall power tubes to a two concentric thin-wall tube design. As a result of this design change no detalled data on tubes 70 and 71 were reported.

13. NM Tube Nos. $72-73$

Ref: Progress Reports NMI-7224, 7225, 7226.

Two prototype thick-wall tubes were fabricated with unalloyed U cores to develop irradiation candidates at SRP or at chalk River in the NRU. Tube 73 was destructively evaluated, and tube 72 was processed and shlpped to Chalk Rlver where it was irradiated in NRU.

As a result of the design change to concentric thin-wall power fuel tubes, development of thick-wall tubes was discontinued with these two tubes. Data on tube 72 are presented below.

A. Description

Th1ck-wall tube, 2.060" OD X 0.297" wall (nominal).

B. Composition

Core: Unalloyed U

Cladding: Zircaloy-2 (30 mils)

End seals: Integral-type Zircaloy-2

C. Dimensions

None reported. 
D. Extrusion Conditions

(Done at American Brass Co.)

No data reported.

E. Heat Treatment

Both tubes were beta-heat treated in a salt pot at SRL while still in their copper extrusion jackets.

F. Autoclave Test

Tube 72 was tested for 4 hours at $345^{\circ} \mathrm{C}$ in water followed by 4 hours at $400^{\circ} \mathrm{C}$ in 1500 psi steam. A lustrous, black film resulted. Tube 73 was not autoclaved.

G. Remarks

Tube 72 had outer clad thinning of $9 \mathrm{mils}$ at the front end of the core as determined by autoradiography.

H. Current Condition

Disposition of tube 72 following irradiation in NRU is unknown.

14. NM Tube Nos, 74A, 74B, 74-76

Ref: (1) Progress reports NMI-7226, 7227, 7228

(2) NMI-7202, 12/27/60, H.F.Sawyer, "Evaluation of Zircaloy-4 Clad Unalloyed Urantum Outer Tube No. 74 Made for Flow-Testing at SRP, Extrusion No. 27944".

(3) NMT-7203, 12/14/60, H.F.Sawyer, "Evaluation of Zircaloy-4 Clad Unalloyed Uranium Outer Thube No. 75 , Extrusion No. 27943, Made for Irradiation at Savannah River".

Five tubes were fabricated in this first group of thin-wall outers $\left(2.060^{\prime \prime}\right.$ oD $x 0.180^{\prime \prime}$ wall) two ( $74 \AA$ and $\left.74 \mathrm{~B}\right)$ to evaluate core preshapes, two full-length $\left(118^{\prime \prime}\right)$ tubes $(74,75)$, and one short (50") tube $(76)$. Tube 74 was used for flow testing and tube 75 irradiated at SRP. Destructive evluation of tube 76 , a VBWR prototype, was done at NM. Data for tubes 74 and 75 are presented below. 


\section{A. Description}

Thin-wall tubes, $2.060^{\prime \prime}$ oD $\mathrm{x} 0.180 "$ wall (nominal).

B. Composition

Core: Unalloyed $U$

Cladding: Zircaloy-4 (25 mils)

End seals: Integral-type zirconium

C. Dimensions

Taken before final bright-etching. (ID should be increased $0.003^{11}$ and OD decreased by $0.003^{\circ}$.)

\begin{tabular}{lcc} 
Over uniform core, OD & $\begin{array}{c}\text { Tube 74 } \\
\text { (1nches) }\end{array}$ & $\begin{array}{c}\text { Tube 75 } \\
\text { (inches) }\end{array}$ \\
\cline { 2 - 3 } ", ID & 2.055 & 2.065 \\
Over end seals, OD & 1.684 & 1.694 \\
", ID & 2.069 & 2.070 \\
Overall length & 1.697 & 1.696 \\
Core, tip-to-t1p & 118 & 118 \\
& 105 & 103.9
\end{tabular}

D. Extrusion Conditions

(Done at Nuclear Metals.)

Tube 74 Tube 75

NM extrusion number

Tool temperature $\left({ }^{\circ} \mathrm{C}\right)$

Billet temperature $\left({ }^{\circ} \mathrm{C}\right)$

Extrusion force, running (tons)

Extrusion force, maximum (tons)

Extrusion constant, running (tsi)

Ram speed (inches/min)

Extrusion ratio(1)

$\begin{array}{cc}27944 & 27943 \\ 480 & 480 \\ 630 & 630 \\ 755 & 795 \\ 1025 & 1100 \\ 15.6 & 16.4 \\ 15 & 15 \\ 15.1 & 15.1\end{array}$

Billets, copper-clad

Copper cutoff

E. Heat Treatment

Tube 74 was not heat treated.

Beta heat treatment of tube 75 in its copper extrusion jacket was performed at Atlas Steels, Itd., Welland, ontario, using a 15-inch ID by 16-foot deep salt bath. The tube was held in the $730^{\circ} \mathrm{C}$ salt for 10 minutes, alrcooled for $5 \frac{1}{2}$ minutes, then water-quenched. The tube was rotated at $15 \mathrm{rpm}$ to help prevent distortion during the operation.

(1) Based on tool sizes. 
E. Autoclave Test

Both tubes were tested for 4 hours at $345^{\circ} \mathrm{C}$ in water followed by 4 hours at $400^{\circ} \mathrm{C}$ in $1500 \mathrm{psi}$ steam. A continuous, hard, lustrous, black oxide film was observed on both tubes.

G. Remarks

The angular preshape used in tube $74 \mathrm{~A}$ produced more uniform cladding in the taper region than the spherical preshapes of tube 74B. Angular preshapes were subsequently used.

H. Current Condition

Tube 74 is stored at SRL and is not a suitable candidate for Irradiation.

15. NM Tube Nos. $77-83$

Ref: (1) Progress Reports NMI-7226, 7227, 7228.

(2) NMI-7204, 11/20/61, W.J.R1chmond, "Evaluation of Zircaloy-4 Clad U-1 w/ S1, Tube No. $79^{\prime \prime}$.

(3) NMI-7212, 12/21/61, W.J.R1chmond, "Evaluation of Zircaloy-4 Clad U-1.5 w/o Mo Tubes No. 82 and No. $83^{\prime \prime}$.

(4) NMI-7212 Supplement, 2/15/63, W.J.Richmond, "Completion of Evaluation of Zircaloy-Clad $\mathrm{U}-1.5 \mathrm{w} / \mathrm{0}$ Mo Tube No. $83^{\prime \prime}$.

To produce VBWR enrlched thin-wall tubes for 1rradiation tests, a group of four natural $U$ prototypes and three enriched U ( $3 \% 235 U)$ were fabricated. One of the natural $U$ tubes (77) and one enriched $U$ tube (79) were alloyed with 1 w/o S1. The remaining three natural $\mathrm{U}$ tubes $(78,80,81)$ and two enriched tubes $(82,83)$ were alloyed with $1.5 \mathrm{w} / \mathrm{O}$ Mo. The four natural $U$ tubes were destructively evaluated. Tubes 79 and 83 are both suitable for irradiation test and are stored at SRL. Thube 82 was Irradiated in the HWCTR. Data for the three enriched $U$ tubes are presented below.

A. Description

Thin-wall tubes, $2.060^{\prime \prime}$ OD $\mathrm{x} 0.180^{\prime \prime}$ wall nominal for the VBWR. 
B. Composition

Core: Tube $77 \quad-\mathrm{U}-1 \mathrm{w} / 0 \mathrm{Si}$

$$
\begin{aligned}
& \text { " } 78,80,81-U-1 \text { - } / 1.5 \mathrm{w} / 0 \text { (U enriched to } 3 \% \text { 235U) } \\
& \text { " } 82,83-U-I .5 \text { W/o Mo (U enriched to } 3 \% \text { 235 U) }
\end{aligned}
$$

Cladding: Zircaloy-4 (25 mils)

End seals: Integral-type zirconium (Zircaloy-4 for tubes 77 and 79 )

C. Dimensions

Taken before final bright-etching. (ID should be increased

\begin{tabular}{|c|c|c|c|}
\hline & \multicolumn{3}{|c|}{ Tube Numbers } \\
\hline & $\begin{array}{c}79 \\
\text { (inches) }\end{array}$ & $\begin{array}{c}82 \\
\text { (inches) }\end{array}$ & $\begin{array}{c}83 \\
\text { (inches) }\end{array}$ \\
\hline Over uniform core, OD & $\begin{array}{l}2.072 \\
1.699\end{array}$ & $\begin{array}{l}2.072 \\
1.695\end{array}$ & $\begin{array}{l}2.071 \\
1.696\end{array}$ \\
\hline$\underset{\|}{\text { Over end seals, }}$ & $\begin{array}{l}2.081 \\
1.707\end{array}$ & $\begin{array}{l}2.080 \\
1.701\end{array}$ & - \\
\hline $\begin{array}{l}\text { Overall length } \\
\text { Core, Tip-to-tip }\end{array}$ & $\begin{array}{l}50 \\
41-3 / 4\end{array}$ & $\begin{array}{l}50 \\
42-1 / 2\end{array}$ & $\begin{array}{l}44-11 / 16 \\
43-1 / 4\end{array}$ \\
\hline
\end{tabular}
$0.003^{\prime \prime}$ and OD decreased by $0.003^{\mathrm{n}}$.)

D. Extmusion Conditions

(Done at American Brass Co.)

NM extrusion number

Tool temperature $\left({ }^{\circ} \mathrm{C}\right)$

Billet temperature $\left({ }^{\circ} \mathrm{C}\right.$

Extmision force, manning (tons)

Tube 79 Tube 82 Tube 83

Extrusion constant, running (tsi)

Ram speed (inches/min)

\begin{tabular}{|c|c|c|}
\hline $\begin{array}{c}28553 \\
430-480(1) \\
649 \\
1280 \\
1360 \\
20.0 \\
25 \\
18.3\end{array}$ & $\begin{array}{c}29115 \\
430-480 \\
632 \\
1120 \\
1200 \\
17.5 \\
25 \\
18.3\end{array}$ & $\begin{array}{c}29116 \\
430-480 \\
632 \\
1140 \\
1320 \\
17.8 \\
25 \\
18.3\end{array}$ \\
\hline
\end{tabular}

Extrusion ratio

Billets copper-clad

Copper cutoff

E. Heat Preatment

Tube 79 was immersed in a triple chloride salt bath at $740^{\circ} \mathrm{C}$ for 10 minutes followed by air-cooling in its copper extrusion jacket.

(1) Liner at $430^{\circ} \mathrm{C}$; die and mandrel at $480^{\circ} \mathrm{C}$. 
Tubes 82 and 83 were each placed in an evacuated steel container in a graphite-lined stainless steel angle support fixture and heat treated at $775^{\circ} \mathrm{C}$ for 15 minutes, furnace-cooled to $535^{\circ} \mathrm{C}$, held 1 hour, then alr-cooled. This heat treatment was developed to give extmuded material a microstmucture resembling that of as-cast material.

F. Autoclave Test

Tubes 79 and 82 were tested for 4 hours at $345^{\circ} \mathrm{C}$ in water followed by 4 hours at $400^{\circ} \mathrm{C}$ in 1500 psi steam. Both tubes exhibited a continuous, hard, black oxide surface.

Tube 83 was tested for 4 hours at $345^{\circ} \mathrm{C}$ in water followed by 32 hours at $400^{\circ} \mathrm{C}$ in $1500 \mathrm{ps} 1$ steam. A continuous, hard, black oxide surface film resulted.

G. Remarks

None.

H. Current Condition

Tubes 79 and 83 are stored at SRL and are suitable for irradiation testing.

16. NM Tube Nos. 84-99, 106, 113

Ref: (1) Progress Reports NMI-7227, 7228, 7229, 7230, 7231, 7232, 7233,7234 .

(2) NMI-7208, 4/24/61, H.F.Sawyer and E.F.Jordan, "Evaluation of the Prototype Set of Zircaloy-4 Clad Unalloyed Uran1um Outer Tubes Nos. 95 and 97, Extrusions No. 28965 and No. $28967 "$ ".

A group of 18 tube extrusions were made to obtain basic information for fabrication of thin-wall outer tubes using unalioyed uranium cores. Fifteen of these tubes were of full length (118") with the three remaining tubes $(98,106,113)$ being of short length (50") for the VBWR.

Two of the long tubes $(95,97)$ were flow tested at SRL; a third (96) was damaged during extrusion; and the remaining 15 tubes were destructively evaluated. Data for tubes 95 and 97 are presented below.

A. Description

Thin-wall tubes, $2.060^{\prime \prime}$ OD X $0.180^{\prime \prime}$ wall (nominal). 
B. Composition

Core: Unalloyed urantum

Cladding: Zircaloy-4 (25 mils)

End seals: Integral-type, zircontum

C. Dimensions

Taken before final bright-etching. (ID should be increased

$0.003^{\prime \prime}$ and $O D$ decreased by $0.003^{\prime \prime}$.)

$\begin{array}{ll}\begin{array}{c}\text { Tube 95 } \\ \text { (inches) }\end{array} & \begin{array}{l}\text { Tube 97 } \\ \text { (inches) }\end{array} \\ 2.060 & 2.062 \\ 1.699 & 1.696 \\ 2.068 & 2.074 \\ 1.699 & 1.701 \\ 118 & 118 \\ 111.6 & 111.0\end{array}$

D. Extrusion Conditions

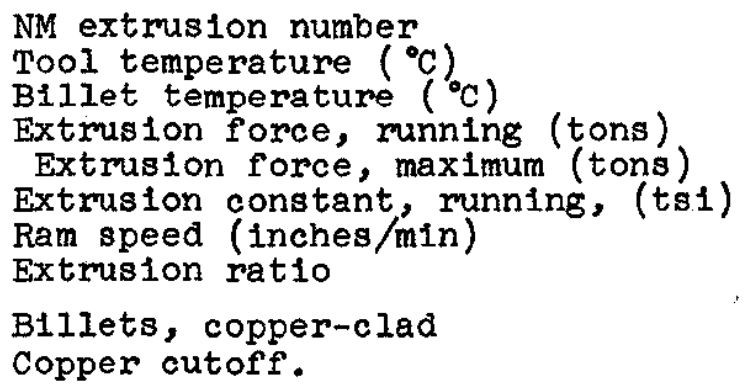

\begin{tabular}{ccc} 
Tube 95 & Tube 97 \\
\cline { 3 - 3 } 28965 & & 28967 \\
480 & & 480 \\
630 & 630 \\
850 & 740 \\
1140 & 985 \\
17.7 & 15.4 \\
15 & 15 \\
15.1 & 15.1
\end{tabular}

E. Heat Treatment

Both tubes 95 and 97 were given two heat treatments in their copper extrusion jackets--the first at SRL and the second at Atlas steels, Itd., Welland, Ontario. The first consisted of 10 minutes in a $735^{\circ} \mathrm{C}$ salt bath and oil quenched for 5 minutes. Subsequent work at NM indicated alr-cooling is preferable to oll-quenching. Therefore, the tubes were given a second heat treatment of 10 minutes in $740^{\circ} \mathrm{C}$ salt, air-cooled for 6 minutes, and then water-quenched for 2 minutes. 
F. Autoclave Test

Tubes 95 and 97 were tested for 4 hours at $345^{\circ} \mathrm{C}$ in water followed by 4 hours at $400^{\circ} \mathrm{C}$ in $1500 \mathrm{ps} 1$ steam. Both tubes exhibited a continuous, hard, black oxide surface.

G. Remarks

This group of 18 tubes resulted in obtaining an acceptable billet design for thin-wall outer tubes for both full-

length tubes and VBWR short-length tubes.

H. Current Condition

Tubes 95 and 97 are stored at SRL and are not considered suitable for irradiation. Tube 96 is stored at NM in the as-extruded condition.

17. NM Tube Nos. 100-105

Ref: (1) Progress Reports NMI-7227, 7229, 7230, 7231, 7232, 7233.

(2) NMI-7206, 3/28/61, W.I.Iarson, "Evaluation of Zircaloy-4 Clad Unalloyed Uranium Inner Tubes Nos, 103 and 104 Made For Irradiation Testing at Savannah River Plant".

(3) NMI-7207, 12/2/60, W.L. Larson, "Evaluation of Zircaloy-4 Clad Unalloyed Uranium Inner Tube No. 105 Made for Flow Testing at SRP".

As part of the development program to produce unalloyed uranium thin-wall inner tubes for the HWCTR, a group of three experimental (100-102) and three prototype (103-105) tubes were fabricated. Th1s tube, 1.020" OD X $0.180^{\prime \prime}$ wall (nomina1), is the inner of two concentric tubes designed to replace the thick-wall single fuel tube design. The three experimental tubes were destructively evaluated. Of the three prototypes, one (105) was used for flow tests at SRL with the other two $(103,104)$ 1rradiated at SRP. Data for the three prototype tubes are presented below.

A. Description

Thin-wall inner tube, $1.020^{\prime \prime}$ OD X $0.180^{\prime \prime}$ wall (nominal). Drawing $3 T-M D X 4-3144$.

B. Composition

Core: Unalloyed uranium

Cladiling: Zircaloy-4 (25 mils)

End seals: Integral-type, zircontum 
C. Dimensions

Taken before final bright-etching. (ID should be increased $0.003^{\prime \prime}$ and OD decreased by $0.003^{\mathrm{N}}$.)

\begin{tabular}{|c|c|c|c|}
\hline & $\begin{array}{l}\text { Tube } 103 \\
\text { (inches) }\end{array}$ & $\begin{array}{l}\text { Tube } 104 \\
\text { (inches) }\end{array}$ & $\begin{array}{l}\text { Tube } 105 \\
\text { (inches) }\end{array}$ \\
\hline Over uniform core, OD & $\begin{array}{l}1.023 \\
0.654\end{array}$ & $\begin{array}{l}1.023 \\
0.654\end{array}$ & 1.021 \\
\hline Over end seals, OD & $\begin{array}{l}1.028 \\
0.656\end{array}$ & $\begin{array}{l}1.028 \\
0.655\end{array}$ & $\begin{array}{l}1.027 \\
0.656\end{array}$ \\
\hline OveraIl length & 118 & 118 & 118 \\
\hline Core, tip-to-tip & $106-1 / 2$ & $106-9 / 16$ & $106-9 / 16$ \\
\hline
\end{tabular}

D. Extrusion Conditions

Tube 103 Tube 104 Tube 105

\begin{tabular}{|c|c|c|c|c|}
\hline $\begin{array}{l}\text { NM extmus } \\
\text { Tool tempe } \\
\text { Biliet tem } \\
\text { Extrusion } \\
\text { Extrusion } \\
\text { Fxtmision }\end{array}$ & $\begin{array}{l}\text { Lon number } \\
\text { rature }\left({ }^{\circ} \mathrm{C}\right) \\
\text { perature }\left({ }^{\circ} \mathrm{C}\right) \\
\text { force, munning (tons) } \\
\text { force, maximum }\end{array}$ & $\begin{array}{l}29309 \\
480 \\
630 \\
458 \\
596\end{array}$ & $\begin{array}{l}20310 \\
480 \\
630 \\
440 \\
617\end{array}$ & $\begin{array}{l}29311 \\
480 \\
630 \\
500 \\
678\end{array}$ \\
\hline Extrusion & constant, running & 16.7 & 16.0 & 8.2 \\
\hline $\begin{array}{l}\text { am speed } \\
\text { xtmusion }\end{array}$ & (Inches/min) & 7.6 & $\begin{array}{l}13 \\
17.6\end{array}$ & \\
\hline
\end{tabular}

Billets, copper-clad

Copper cutoff

E. Heat Treatment

Tubes 103 and 104 were beta-treated at Atlas Steels, Itd., Welland, Ontario, in their copper extmusion jackets. The tubes were rotated at $15 \mathrm{rpm}$ during the heat treating cycle to minimize warpage. The cycle consisted of (1) 10 minutes In $740^{\circ} \mathrm{C}$ salt; (2) air-cooling for 6 minutes; and (3)

quenching in $20^{\circ} \mathrm{C}$ water for 2 minutes.

No heat treatment was given to tube 105.

F. Autoclave Test

All three tubes were tested for 4 hours at $345^{\circ} \mathrm{C}$ in water followed by 4 hours at $400^{\circ} \mathrm{C}$ in $1500 \mathrm{ps} 1$ steam. The tubes all exhibited a continuous, hard, black oxide surface.

G. Remarks

None.

H. Current Condition

Tobe 105 is stored at SRL; it is not sultable for irradiation. 
18. NM Tube Nos. 107-112

Ref: (1) Progress Reports NMI-7230, 7232, 7233, 7234.

(2) NMI-7209, 5/2/6I, H.F.Sawyer and E.F.Jordan, "Evaluation of the Demonstration Set of Zircaloy-4 Clad Unalloyed Uranium Outer Tubes Nos. 108-112 Extrusion Nos. 29778 to 29782 ."

As a follow-up to the 18 experimental thin-wall outer tubes (Item 16), a demonstration set of $s 1 x$ full-length tubes was fabricated. The first tube of this set (107) was destructively evaluated and appeared to meet ali specifications. Based on this, the remaining five billets were machined, assembled and extruded. Tube 108 was irradiated at SRP and tube 111 was used for flow tests. Data for tubes 108-112 are presented below.

A. Description

Thin-wall tubes, $2.060^{\prime \prime}$ OD X $0.180^{\prime \prime}$ wall (nominal).

B. Composition

Core: Unalloyed uranlum

Cladding: Zircaloy-4 (25 mils)

End seals: Integral-type, zirconium

C. Dimensions

Taken before final bright-etching. ID should be increased $0.003^{\prime \prime}$ and OD decreased by $0.003^{\pi}$.)

Tube Numbers

\begin{tabular}{|c|c|c|c|c|}
\hline $\begin{array}{l}108 \\
\text { (in.) }\end{array}$ & $\begin{array}{l}109 \\
(\text { in.) }\end{array}$ & $\begin{array}{l}\text { IIo } \\
\text { (in.) }\end{array}$ & $\begin{array}{l}I_{1} \\
(\mathrm{In.})\end{array}$ & $\begin{array}{l}\text { II2 } \\
\text { (in.) }\end{array}$ \\
\hline $\begin{array}{l}2.059 \\
1.695\end{array}$ & $\begin{array}{l}2.061 \\
1.694\end{array}$ & $\begin{array}{l}2.059 \\
1.696\end{array}$ & $\begin{array}{l}2.057 \\
1.694\end{array}$ & $\begin{array}{l}2.060 \\
1.693\end{array}$ \\
\hline $\begin{array}{l}2.071 \\
1.698\end{array}$ & $\begin{array}{l}2.067 \\
1.706\end{array}$ & $\begin{array}{l}2.069 \\
1.699\end{array}$ & $\begin{array}{l}2.065 \\
1.693\end{array}$ & $\begin{array}{l}2.070 \\
1.700\end{array}$ \\
\hline 118 & 118 & 118 & 118 & 118 \\
\hline 110.4 & 109.7 & 111.1 & 111.4 & 110 \\
\hline
\end{tabular}

D. Extrusion Conditions

NM extrusion number

Tool temperature $\left({ }^{\circ} \mathrm{C}\right)$

Billet temperature $\left({ }^{\circ} \mathrm{C}\right)$

Extrusion force, muning (tons)

\begin{tabular}{|c|c|c|c|c|}
\hline 108 & 109 & 110 & III & 112 \\
\hline $\begin{array}{c}29778 \\
480\end{array}$ & $\begin{array}{c}29779 \\
480\end{array}$ & $\begin{array}{c}29780 \\
480\end{array}$ & $\begin{array}{c}29781 \\
480\end{array}$ & $\begin{array}{c}29782 \\
480\end{array}$ \\
\hline
\end{tabular}

$630 \quad 630 \quad 630 \quad 630 \quad 630$

$805 \quad 835 \quad 725 \quad 770 \quad 825$

Extrusion constant, munning (ts1) $\begin{array}{cccccc}1050 & 1050 & 17.6 & 970 & 980 & 1050\end{array}$

Ram speed (inches/min)

Extrusion ratio

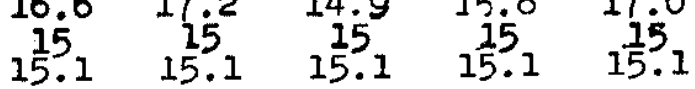

Billets, copper-clad

Copper cutoff 
E. Heat Treatment

A11 five tubes were beta-treated at Atlas Steels, Ltd., Welland, Ontario, in their copper extrusion jackets. The tubes were rotated at $15 \mathrm{rpm}$ during the heat treating cycle. The cycle consisted of (1) 10 minutes in $740^{\circ} \mathrm{C}$ salt; (2) air cooling for 6 minutes; and (3) quenching in $20^{\circ} \mathrm{C}$ water for 2 minutes.

F. Autoclave Test

The tubes were tested for 4 hours at $345^{\circ} \mathrm{C}$ in water followed by 4 hours at $400^{\circ} \mathrm{C}$ in $1500 \mathrm{psi}$ steam. All tubes exhibited a continuous, hard, black oxide surface.

G. Remarks

None.

H. Current Condition

Tube 111 is stored at SRL and is not considered suitable for Irradiation. Tubes 109,110 and 112 are stored at NM with only 110 considered sultable for irradiation.

19. NM Tube Nos. 114-115

Ref: (1) Progress Reports NMI-7233, 7234, 7235.

(2) NMI-7213, 4/2/62, W.J.RIchmond, "Evaluation of ZircaloyClad Uranium Tubes Nos. 114 and $115 "$.

Two prototype, enriched, thin-wall tubes for VBWR were fabricated. Both tubes are sultable for Irradiation and are stored at SRL. Data for these tubes are presented below.

\section{A. Description}

Thin-wall tubes, $2.060^{\prime \prime}$ OD X $0.180^{\prime \prime}$ wall (nominal) for the VBWR.

B. Composition

Core: Unalloyed urantum enriched to $3 \% 235 \mathrm{U}$.

Cladding: Zircaloy-2 (25 mils)

End seals: Integral-type, zirconium. 
C. Dimensions

Taken before final bright-etching. (ID should be increased $0.003^{\prime \prime}$ and OD decreased by $0.003^{\pi}$.)

\begin{tabular}{|c|c|}
\hline $\begin{array}{l}\text { Tube } 114 \\
\text { (inches) }\end{array}$ & $\begin{array}{l}\text { Tube } 115 \\
\text { (1nches) }\end{array}$ \\
\hline $\begin{array}{l}2.062 \\
1.693\end{array}$ & $\begin{array}{l}2.061 \\
1.692\end{array}$ \\
\hline $\begin{array}{l}2.072 \\
1.697\end{array}$ & $\begin{array}{l}2.073 \\
1.697\end{array}$ \\
\hline $\begin{array}{c}50 \\
44-1 / 4\end{array}$ & $\begin{array}{c}50 \\
44-3 / 32\end{array}$ \\
\hline
\end{tabular}

D. Extrusion Conditions

Tube 114 Tube 115

NM extrusion number

Tool temperature $\left({ }^{\circ} \mathrm{C}\right)$

Billet temperature $\left({ }^{\circ} \mathrm{C}\right)$

Extrusion force, munning (tons)

Extrusion constant, running ( $t s 1$ )

Ram speed (1nches/min)

Extrusion ratio

$30103 \quad 30104$

$480 \quad 480$

$630 \quad 630$

$800 \quad 770$

$1000 \quad 1005$

$17.3, \quad 16.7$

$\begin{array}{ll}15 & 15 \\ 15.1 & 15.1\end{array}$

B1llets copper-clad

Copper cutoff

E. Heat Treatment

Both tubes were beta treated in their copper extrusion jackets and consisted of 10 minutes in $740^{\circ} \mathrm{C}$ salt followed by air cooling.

F. Autoclave Test

The tubes were tested for 4 hours at $345^{\circ} \mathrm{C}$ in water followed by 4 hours at $400^{\circ} \mathrm{C}$ in $1500 \mathrm{ps} 1$ steam. Both tubes exhibited a continuous, hard, black oxide surface.

G. Remarks

None.

H. Current Condition

Both tubes are stored at SRL and are considered suitable for irradiation. 
20. NM Tube Nos. 116-121

Ref:(I) Progress Reports NMI-7234, 7235, 7236.

(2) NMI-7210, 8/11/61, W.I.Larson, "Evaluation of the Demonstration Set of Zircaloy-4-Clad Unalloyed Uranium Inner Tubes Nos. 116-121, Extmusion Nos. 30795 to 30800 "

This group of $\mathrm{six}$ thin-wall inner tubes with unalloyed uranium cores and of identical design were made to demonstrate the reproducibility of the fabrication process. One of the tubes (121) was processed as a flow test tube and was not heat treated. The other five were beta-treated and processed as candidates for irradiation testing. Two of these tubes (117, 119) were Irradiated at SRP. Data for the tubes are presented below.

A. Description

Thin-wall inner tubes, $1.020^{\prime \prime}$ OD $\mathrm{X} 0.180 "$ wall (nominal).

B. Composition

Core: Unalloyed uranium

Cladding: Zircaloy-4 (25 mils)

End seals: Integral-type, zirconium.

C. Dimensions

Taken before final bright-etching. (ID should be increased $0.003^{\prime \prime}$ and $O D$ decreased by $0.003^{\pi}$.)

Over uniform core,
Over end seals, ${ }_{11}$,
Overall length

Core, tip-to-tip

D. Extrusion Conditions

$N M$ extrusion number

Tool temperature $\left({ }^{\circ} \mathrm{C}\right)$

Billet temperature $\left({ }^{\circ} \mathrm{C}\right)$

Extrusion force, running (tons)

Extrusion constant, running(ts1)

Ram speed (inches/min)

Extrusion ratio

Blilets copper-clad

Copper cutoff
Tube Numbers

\begin{tabular}{|c|c|c|c|c|c|}
\hline$\left(\begin{array}{ll}116 \\
(\text { in. })\end{array}\right.$ & 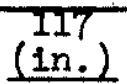 & $\begin{array}{c}118 \\
\text { (in.) }\end{array}$ & $\begin{array}{c}119 \\
\text { (in.) }\end{array}$ & $\begin{array}{l}120 \\
(\ln .)\end{array}$ & $\begin{array}{l}121 \\
\text { (in.) }\end{array}$ \\
\hline $\begin{array}{l}1.026 \\
0.654 \\
1.032 \\
0.655\end{array}$ & $\begin{array}{l}0.653 \\
1.031 \\
0.654 \\
118 \\
109.4\end{array}$ & $\begin{array}{l}1 . \\
0 . \\
1 . \\
0 . \\
11\end{array}$ & & 10.9 & \\
\hline \multicolumn{6}{|c|}{ Tube Numbers } \\
\hline 116 & 117 & & & 120 & 121 \\
\hline $\begin{array}{l}20795 \\
480 \\
630 \\
596 \\
767 \\
21.8 \\
13\end{array}$ & $\begin{array}{c}30796 \\
480 \\
630 \\
535 \\
725 \\
19.6 \\
13 \\
17.6\end{array}$ & $\begin{array}{c}30797 \\
480 \\
630 \\
435 \\
587 \\
15.9 \\
13 \\
17.6\end{array}$ & $\begin{array}{c}30798 \\
480 \\
630 \\
445 \\
623 \\
16.2\end{array}$ & $\begin{array}{l}30799 \\
480 \\
630 \\
475 \\
689 \\
17.4 \\
13 \\
17.6\end{array}$ & $\begin{array}{r}30 \\
4 \\
6 \\
5 \\
7\end{array}$ \\
\hline
\end{tabular}


E. Heat Treatment

Tube 121 was not treated.

Tubes 116 through 120 were beta-treated at Atlas Steels, Itd., Welland, Ontario. The cycle was as follows:

(1) 10 minutes in $730^{\circ} \mathrm{C}$ salt; (2) 6 minutes alr cooling; and (3) water quenching $\left(20^{\circ} \mathrm{C}\right)$ for 2 minutes. The tubes were rotated at $15 \mathrm{rpm}$ during heat treatment in their copper extasion jackets.

F. Autoclave Test

Tubes 116,118 and 120 were not tested.

Tubes 117,119 and 121 were tested for 4 hours at $345^{\circ} \mathrm{C}$

in water followed by 4 hours at $400^{\circ} \mathrm{C}$ in $1500 \mathrm{ps} 1$ steam.

The tubes exhibited a continuous, hard, black oxide surface.

G. Remarks

Tubes 120 and 121 were used for flow tests at SRL.

H. Current Condition

Tubes 116 and 118 are stored at NM and tubes 120 and 121 are stored at SRL. The first two tubes $(116,118)$ have been etched but not autoclaved and may be candidates for irradiation.

21. NM Tube Nos. $122-124$

Ref: Progress Reports NMI-7236, 7237, 7238, 7240.

A group of three experimental tubes was fabricated to improve the quality of unalloyed uranium thin-wall outer tubes.

Evaluation of two of these tubes $(122,123)$ showed that more extensive alpha working of the billet core stock results in more uniform cladding and smoother interface on the extruded tube. This was subsequently confirmed by extrusion of a third tube (124). No detailed dimensional data on evaluation of these tubes were reported.

22. NM Tube Nos. 125-127

Ref: (I) Progress Reports NMI-7240, 7241, 7242.

(2) NMI-7214, 8/24/62, W.J.Richmond, "Evaluation of Z1rcaloy-Clad Unalloyed Urantum Outer Tubes Nos. 125, 126 and 127. 
This demonstration set of three unalloyed uranium thin-wall outer tubes were fabricated to evaluate the process for producing the outer tubes for the injtial loading of the HWCTR. Data for these tubes are presented below.

A. Description

Thin-wall tubes, $2.060^{\prime \prime}$ OD X $0.180^{\prime \prime}$ wall (nominal) for the HWCTR.

B. Composition

Core: Unalloyed uranium

Cladding: Nickel-free Zircaloy-2 (25 mils)

End Seals: Integral-type, zircontum

c. Dimensions

\begin{tabular}{|c|c|c|c|}
\hline 年 & $\begin{array}{l}\text { Tube } 125 \\
\text { (1nches) }\end{array}$ & $\begin{array}{l}\text { Tube } 126 \\
\text { (inches) }\end{array}$ & $\begin{array}{l}\text { Tube } 127 \\
\text { (inches) }\end{array}$ \\
\hline Over uniform core, OD & $\begin{array}{l}2.056 \\
1.697\end{array}$ & $\begin{array}{l}2.055 \\
1.696\end{array}$ & $\begin{array}{l}2.055 \\
1.695\end{array}$ \\
\hline Over end seals, OD & $\begin{array}{l}2,062 \\
1.699\end{array}$ & $\begin{array}{l}2.062 \\
1.698\end{array}$ & $\begin{array}{l}2.062 \\
1.700\end{array}$ \\
\hline Overall length & 118.0 & 118.0 & 118.0 \\
\hline Core, tip-to-tip & 110.6 & 109.6 & 109.5 \\
\hline
\end{tabular}

D. Extrusion Conditions

Tube 125 Tube 126 Tube 127

NM extrusion number

Tool temperature $\left({ }^{\circ} \mathrm{C}\right)$

Billet temperature $\left({ }^{\circ} \mathrm{C}\right)$

Extrusion force, munning (tons)

Extrusion constant, running (ts1) 16.4

Ram speed (Inches/min)

Extrusion ratio

32001

370
630

790

1000

Billets copper-clad

15

15.1

32002

370
630

32003

370
630

780

950

16.1

15

15.1

780

970

16.1

15

15.1

Copper cutoff

E. Heat Treatment

The tubes were beta-treated in their copper extrusion jackets at Atlas Steels, Ltd. Each tube was immersed for 10 minutes in $740^{\circ} \mathrm{C}$ salt, air-cooled for 6 minutes, then water quenched while being rotated at $15 \mathrm{rpm}$. 
F. Autoclave Test

All of the tubes were tested for 4 hours at $345^{\circ} \mathrm{C}$ in water followed by 4 hours at $400^{\circ} \mathrm{C}$ in 1500 psi steam. Each of the three tubes exhibited a continuous, hard, black oxide surface.

G. Remarks

Tubes 126 and 127 meet all specifications for imradiation candidates. Tube 125 was satisfactory in all respects except overall bow which was $5 / 32$ inch (1/8 maximum spec1fled). Th1s tube was later stralghtened to a bow of $1 / 16^{\prime \prime}$.

H. Current Condition

All three tubes are stored at NM and are suitable for irradiation.

23. NM Tube Nos. 128-129, 136

Ref: (I) Progress Reports NMI-7239, 7240, 7241, 7242, 7243, $7244,7247$.

(2) NMI-7215 DE, $7 / 16 / 62$, E.F.Jordan, "Evaluation of Restraint Tubes 128, 129 and $136^{\prime \prime}$.

A group of three thin-wall inner tubes having unalloyed uranium cores was fabricated to evaluate the effect of cladding restraint on fuel swelling during irradiation. Tubes 128 and 136 are identical in design, with $60 \mathrm{mils}$ cladding as compared with the nominal 25 mils normally used. Tube 129 has 40 mils outside and 25 mils inside cladding. Tube 136 was fabricated as a substitute for 128 which had a striation on the outside cladding making it unsuitable for irradiation. Data for the three tubes are presented below.

A. Description

Thin-wall restraint inner tubes.

Type D: $(128,136), 1.020^{\prime \prime}$ OD X 0.520" ID, $0.130^{\prime \prime}$ core, $0.060^{\prime \prime}$ outer and $0.060^{\prime \prime}$ inner clad.

Type E: (129), 1.020" OD X 0.630" ID, $0.130^{\prime \prime}$ core, $0.040 "$ outer and $0.025^{\prime \prime}$ inner clad.

B. Composition

Core: Unalloyed uranium

Cladding: Nickel-free Zircaloy-2 (thickness as above)

End seals: Integral-type, zirconium 
C. Dimensions

Over uniform core, OD

Over end seals, OD

Overall length

Core, tip-to-tip

D. Extrusion Conditions

NM extrusion number

Tool temperature $\left({ }^{\circ} \mathrm{C}\right)$

Billet temperature $\left({ }^{\circ} \mathrm{C}\right)$

Extrusion force, munning (tons)

Extrusion constant, running (tsi)

Ram speed (inches/min)

Extrusion ratio

Billets copper-clad

Copper cutoff

E. Heat Treatment

Tubes 128 and 129 were heat treated at Atlas Steels, Itd. with tube 136 done at Watertown Arsenal. Each tube was beta treated in its copper extrusion jacket while being rotated at $17 \frac{1}{2} \mathrm{rpm}$. Treatment consisted of immersion in a $730^{\circ} \mathrm{C}$ salt bath for 10 minutes, air-cooled for 6 minutes, and waterquenched for 2 minutes.

F. Autoclave Test

Tube 129 was tested for 4 hours at $345^{\circ} \mathrm{C}$ in water followed by 4 hours at $400^{\circ} \mathrm{C}$ in $1500 \mathrm{ps} 1$ steam. Tubes 128 and 136 underwent a similar test but were held for 32 hours in steam. All tubes exhibited the black oxide film associated with Zircaloy having good aqueous corrosion resistance, except for tube 128 which exhibited localized white oxide in the heavily-scored section of the outside cladding.

G. Remarks

None.

H. Current Condition

Tube 128 is stored at NM and is not considered suitable for irradiation. Tubes 129 and 136 are stored at SRL and are both suitable for irradiation.

\begin{tabular}{c|cc}
$\begin{array}{c}\text { Tube 128 } \\
\text { (1nches) }\end{array}$ & $\begin{array}{c}\text { Tube 136 } \\
\text { (1nches) }\end{array}$ & $\begin{array}{c}\text { Tube 129 } \\
\text { (1nches) }\end{array}$ \\
\cline { 1 - 2 } 1.015 & 1.016 & 1.019 \\
0.523 & 0.520 & 0.630 \\
1.030 & 1.020 & 1.028 \\
0.522 & 0.520 & 0.629 \\
118 & 118 & 118 \\
105.2 & 107.1 & 108.0
\end{tabular}

Tube 128 Tube 136 Tube 129

$\begin{array}{ccc}32039 & 33158 & 32040 \\ 480 & 370 & 480 \\ 630 & 630 & 630 \\ 519 & 392 & 551 \\ 689 & 519 & 731 \\ 20.2 & 15.2 & 20.4 \\ 13 & 13 & 13 \\ 14.3 & 14.3 & 16.8\end{array}$


24. NM Tube Nos. 130-133

Ref: (1) Progress Reports NMI-7239, 7240, 7241, 7242, 7243, $7244,7247$.

(2) NMI-7215 BC, 6/20/62, E.F.Jordan, "Evaluation of Restraint Tubes 130 and $131 "$.

(3) NMI-7215-A, 6/14/62, E.F.Jordan, "Evaluation of Restraint Tubes 132 and $133^{\prime \prime}$.

A group of four thin-wall outer tubes having unalloyed uranium cores were fabricated to evaluate the effect of cladding restraint on fuel sweliling during irradiation. Tubes 132 and 133 had 60 mils outer and inner clad; tube 130,25 mil outer and 40 mil inner; tube 131, $40 \mathrm{mil}$ outer and $25 \mathrm{mil}$ inner. Tube 132 was not considered an irradiation candidate because of white oxide formation during autoclaving; tube 133 was irradiated in HWCTR. Data for the four tubes are presented below.

A. Description

Thin-wall restraint outer tubes.

Type A: $(132,133), 2.060^{\prime \prime}$ oD X 1.560" ID, $0.130^{\prime \prime}$ core, $0.060^{\prime \prime}$ outer and $0.060^{\prime \prime}$ inner clad.

Type B: (130), 2,060" OD X 1.670" ID, 0.130" core, $0.025^{\prime \prime}$ outer and $0.040^{\prime \prime}$ inner clad.

Type C: (131), 2.060" on X 1.670" ID, 0.130" core, $0.040^{\prime \prime}$ outer and $0.025^{\prime \prime}$ inner clad.

B. Composition

Core: Unalloyed urantum

Cladding: Nickel-free Zircaloy-2 (thickness as above)

End Seals: Integral type, zirconium.

C. Dimensions

Over uniform core, "D

Over end seals, OD

Overall length

core, t1p-to-t1p
Tube 132 Tube 133 (inches) (Inches) 2.068 1.567

2.072

1.566

118

108.3
2.070
1.568

2.075

1.567

118

107.8
Tube 130 Tube 131 (inches) (inches)

2.052

1.667

2.055

2.061

1.671

1.671

2.062

1.670

118 109.2
118 109.8 


\section{Extrusion Conditions}

Tubes 132 and 133 were extruded at American Brass Co.

Tube 132 Tube 133 Tube 130 Tube 131

\begin{tabular}{|c|c|c|c|c|}
\hline $\begin{array}{l}\text { NM extrusion number } \\
\text { Tool temperature }\left({ }^{\circ} \mathrm{C}\right) \\
\text { Billet temperature }\left({ }^{\circ} \mathrm{C}\right) \\
\text { Extrusion force, munning (tons) } \\
\text { " } \\
\text { Extrusion constant, munning (tsi) } \\
\text { Ram speed (inches/min) } \\
\text { Extrusion ratio }\end{array}$ & $\begin{array}{l}32995 \\
480 \\
630 \\
1200 \\
1400 \\
18.6 \\
25 \\
14.3\end{array}$ & $\begin{array}{l}32996 \\
480 \\
630 \\
1160 \\
1 \overline{8.0} \\
25 \\
14.3\end{array}$ & $\begin{array}{c}32345 \\
370-480(1) \\
630 \\
765 \\
935 \\
16.1 \\
15 \\
14.2\end{array}$ & $\begin{array}{c}32346 \\
370-480(1) \\
630 \\
755 \\
890 \\
15.9 \\
15 \\
14.2\end{array}$ \\
\hline $\begin{array}{l}\text { Billet copper-clad } \\
\text { Copper cutoff }\end{array}$ & & & & \\
\hline
\end{tabular}

(1) Dle and liner at $370^{\circ} \mathrm{C}$; mandrelland cutof $f$ at $480^{\circ} \mathrm{C}$.

E. Heat Treatment

All four tubes were beta treated in their copper extrusion jackets at Atlas Steels, Ltd. The tubes were immersed in $730^{\circ} \mathrm{C}$ salt for 10 minutes, air-cooled for 6 minutes, and water-quenched for 2 minutes. Tubes 130 and 131 were rotated at $15 \mathrm{rpm}$ and tubes 132 and 133 at $17 \frac{1}{2} \mathrm{rpm}$ during heat treatment.

F. Autoclave Test

The tubes were tested for 4 hours at $345^{\circ} \mathrm{C}$ in water followed by 4 hours $(130,131)$ or 32 hours $(132,133)$ at $400^{\circ} \mathrm{C}$ in $1500 \mathrm{ps} 1 \mathrm{steam}$. Tube 132 exh1bited white oxide, indicative of excessive corrosion, on both the outside and inside surfaces between 18 and 36 inches from the rear of the tube. The remaining three tubes all exhibited a continuous black oxide surface film associated with good corrosion resistance in water.

G. Remarks

None.

H. Current Condition

Tubes 130 and 131 are stored at SRI and are considered suitable for irradiation. Tube,132, stored at NM, is not an irradiation candidate. 
25. NM Tube Nos. 134-135, 141-142

Ref: (I) Progress Reports NMI-7241, 7242, 7243, 7244, 7247, 7249, 7250, 7251, 7252.

(2) NMI-7217, 6/29/62, W.J.RIchmond, "Evaluation of Zircaloy-Clad U-0.3 w/o Al-0.5 w/0 Si Tube No. 135".

(3) NMI-7219, 1/15/63, W.J.Richmond, "Evaluation of Zircaloy-clad U-1 w/o SI Tube No. $141^{\prime \prime}$.

To obtain thin-wall outer tubes containing cores of afferent uranium alloys that might be superlor to unalloyed uranium in resistance to deformation during irradiation, a group of four tubes with three different core alloys were fabricated. Two tubes (134, 141) contained cores of U-1 w/O S1 with tube 134 being scrapped following stalling during extrusion and tube 135 being processed as an irradiation candidate. Tube 135 contalned a core of U-0.3 w/O Al-0.5 w/O S1 and tube 142 a core of U-1.5 w/o Mo, with 135 being processed as an Irradiation candidate and 142 processed only through extrusion. Data for the three types of tubes are presented below.

A. Description

Thin-wall outer tubes, $2.060^{\prime \prime}$ OD $X 0.180^{\prime \prime}$ wall (nominal).

B. Composition

Core: 134 and $141-U-1$ w/o S1.

$135-\mathrm{U}-0.3 \mathrm{w} / 0$ Al $-0.5 \mathrm{w} / \mathrm{o} \mathrm{S1}$

$142-\mathrm{U}-1.5 \mathrm{w} / \mathrm{O}$ Mo

Cladding: Nickel-free Z1rcaloy-2 (25 m1ls)

End seals: Integral-type, nickel-free Zircaloy-2

C. Dimensions

Over uniform core, OD

Over end seals, OD

Overall length

Core, tip-to-tip

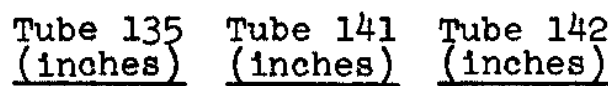

2.065

1.698

2.054

--

2.076

1.700

$-$

1.706

2.064

118

118

$--$

112.1

115.1

118

(113 spec.) 
D. Extrusion Conditions

This group of tubes was extmuded at American Brass Co.

Tube 135 Tube 141 Tube 142

NM extrusion number

Tool temperature $\left({ }^{\circ} \mathrm{C}\right)$

Billet temperature $\left({ }^{\circ} \mathrm{C}\right)$

$\underset{\|}{\text { Extmusion force, muning (tons) }}$

\begin{tabular}{c|cc}
32998 & 35897 &.-- \\
$430-480(1)$ & $430-480$ & $430-480$ \\
650 & 650 & 630 \\
1304 & 1301 & 1134 \\
1672 & 1321 & 1374 \\
20.3 & 20.2 & 17.6 \\
25 & 25 & 25 \\
18.3 & 18.8 & 18.8
\end{tabular}

Extrusion constant, munn
Ram speed (inches/min)

Extrusion ratio

18.3

18.8

18.8

Billets copper-clad

Copper cutoff

(1) Linner at $430^{\circ} \mathrm{C}$; die, mandrel and cut of $f$ at $480^{\circ} \mathrm{C}$.

E. Heat Treatment

Tube 135 and 141 were beta treated in their copper extmusion jackets at Watertown Arsenal. The tubes were immersed in $740^{\circ} \mathrm{C}$ salt for 10 minutes, air-cooled for 6 minutes, and water-quenched for 2 minutes. They were both rotated at $17 \frac{1}{2} \mathrm{rpm}$ during treatment to minimize warpage. Tube 142 was not heat treated.

F. Autoclave Test

The tubes were tested for 4 hours at $345^{\circ} \mathrm{C}$ in water followed by 32 hours in 1500 psi steam. Both tubes exhibited a continuous, black surface film after the test. Tube 142 was not tested.

G. Remarks

None.

H. Current Condition

Tubes 135 and 142 are stored at SRL and are considered suitable for irradiation. Tube 142 is stored at $N M$ in the as-extruded condition. 
26. NM Tubes Nos. $137,143-147$

Ref: (I) Progress Reports NMI-7248 and 7249.

(2) NMI-7220-1, 3/15/63, A.B.Bremer, "Evaluation of Five Inner Tubes of Zircaloy-Clad Enriched Uranium Tubes Nos. 143-147".

This group of thin-wall inner tubes consisted of one prototype with a natural uranium core and five enriched uranium (2.1\% 235v) tubes for Irradiation tests in HWCTR. The prototype (137) was extruded to provide information on billet design and also to provide nonbond standards for ultrasonic testing. All five of the enriched tubes (143-147) met the speciflcations for irradiation testing. Data for these tubes are presented below.

\section{A. Description}

Thin-wall inner tubes, $1.020^{\prime \prime}$ OD X $0.180^{\prime \prime}$ wall (nominal).

B. Composition

Core: Tube 137 - Unalloyed U (natura I)

Tubes 143-147 - Unalloyed U enriched to $2.1 \% 235 \mathrm{~J}$.

Cladding: Nickel-free Zircaloy-2 (25 mils)

End seals: Integral-type, zirconium

C. Dimensions

Tube 143 Tube 144 Tube 145 Tube 146 Tube 147 (inches) (Inches) (Inches) (Inches) (inches)

$\begin{array}{lccccc}\text { Over uniform core, OD } & 1.021 & 1.020 & 1.020 & 1.021 & 1.021 \\ \text { "1 , ID } & 0.660 & 0.658 & 0.659 & 0.659 & 0.660 \\ \text { Over end seals, OD } & - & - & - & - & - \\ " \text { ", ID } & - & - & - & - & - \\ \text { Overall length } & 118 & 118 & 118 & 118 & 118 \\ \text { Core, tip-to-tip } & 109.4 & 109.0 & 109.0 & 108.7 & 108.9\end{array}$

D. Extrusion Conditions

NM extrusion number

Tool temperature $\left({ }^{\circ} \mathrm{C}\right)$

Billet temperature $\left({ }^{\circ} \mathrm{C}\right)$

Extmusion force, munning (tons)

Extmusion constant, running (ts1)

Ram speed (Inches/min)

Extrusion ratio

Tube Numbers

\begin{tabular}{|c|c|c|c|c|}
\hline 143 & 144 & 145 & 146 & 147 \\
\hline $\begin{array}{c}35929 \\
370 \\
630 \\
425 \\
615 \\
15.5 \\
13-15 \\
17.7\end{array}$ & $\begin{array}{c}35930 \\
370 \\
630 \\
500 \\
680 \\
18.2 \\
13-15 \\
17.7\end{array}$ & $\begin{array}{c}35931 \\
370 \\
630 \\
450 \\
590 \\
16.4 \\
13-15 \\
17.7\end{array}$ & $\begin{array}{c}35932 \\
370 \\
630 \\
460 \\
605 \\
16.8 \\
13-15 \\
17.7\end{array}$ & $\begin{array}{c}35933 \\
370 \\
630 \\
490 \\
660 \\
17.9 \\
13-15 \\
17.7\end{array}$ \\
\hline
\end{tabular}

Billet copper-clad

Copper cutoff 
E. Heat Treatment

Tubes 143 and $145-147$ were beta treated at SRL in their copper extrusion jackets. The tubes were immersed in a $740^{\circ} \mathrm{C}$ salt bath for 10 minutes and oll-quenched

for 20 minutes. Tube 144 was beta treated at Watertown Arsenal in a $740^{\circ} \mathrm{C}$ salt bath for 10 minutes, air-cooled for 6 minutes and quenched in water for 2 minutes while being rotated at $15 \mathrm{rpm}$. (The other tubes were not rotated.)

F. Autoclave Test

The tubes were tested for 4 hours at $345^{\circ} \mathrm{C}$ in water followed by 32 hours at $400^{\circ} \mathrm{C}$ in $1500 \mathrm{psi}$ steam. All tubes exhibited a continuous, lustrous, black surface film.

G. Remarks

Nonbonds on the prototype tube (137) were obtained on the outside core-cladding interface by interposing either mica or uranium oxide. To accommodate these nonbond materials in the billet, two $\frac{1}{2}-1$ inch wide recesses $0.060^{\prime \prime}$ deep for the mica and $0.015^{\prime \prime}$ deep for the uranium oxide, were machined in the uranium core. A strip of outgassed mica 0.5 -inch wide $X 0.0025$-inch thlck was wrapped 8.7 turns into the $0.060^{\prime \prime}$ recess to produce a $0.004^{\prime \prime}$ thick $X 7.5^{\prime \prime}$ long layer in the extruded tube. The uranium oxide was produced by torch-oxidizing the surface of the $0.015^{\prime \prime}$ recess.

H. Current Condition

The flve enriched tubes are stored at SRL and are all suitable for irradiation.

27. NM Tubes Nos $138-140$

Ref: Progress Report NMI-7249.

Three thin-wall outer tubes (138-140) were extruded to qualify a simplified process for preparing fine-grain unalloyed uranium cores. The new process consisted of beta treatment followed by a single extrusion at low reduction as compareed to the lowyield process of repreated alpha extrusions with intermediate upsets. (known as Process B). Data for these tubes are presented below.

A. Description

Thin-wall outer tubes, $2.060 "$ OD X $0.180 "$ wall (nominal). 
B. Composition

Core: Unalloyed urantum

Cladding: Nickel-free Zircaloy-2 (25 mils)

End seals: Integral-type, zirconium

C. Dimensions

Not reported.

D. Extrusion Conditions

Tube 138 Tube 139 Tube 140

NM extrusion number

Tool temperature $\left({ }^{\circ} \mathrm{C}\right)$

Billet temperature $\left({ }^{\circ} \mathrm{C}\right)$

35820

35821

35822

Extrusion force, munning (tons)

370

630

370

370

Extrusion force, maximum (tons)

810

630

630

Extmusion constant, running (tsi)

970

830

855

16.7

Ram speed (inches/min)

15

Extmusion ratio

15.0

1015

1080

17.1

17.6

15

15

Billets copper-clad

Copper cutoff

E. Heat Treatment

None.

F. Autoclave Test

None

G. Remarks

All three tubes were destructively examined. The simplified core preparation process resulted in tubes having core-cladding interfaces as smooth as in tubes with cores prepared by the alpha extrusion-intermediate upset process. 
28. NM Tubes Nos. 148-149

Ref: NMI-7220-2, 3/15/63, A.B.Bremer, "Evaluation of Two Outer Tubes of Zircaloy-Clad Enriched Unalloyed Uranium Tube Nos. 148 and 149 ".

Two thin-wall outer tubes with unalloyed uranium enriched to $2.1 \% 235 \mathrm{~J}$ were fabricated for irradiation tests which were done in the HWCTR. Data for these two tubes are presented below.

A. Description

Thin-wall outer tubes, $2.060^{\prime \prime}$ OD $\times 0.180$ " wall (nominal).

B. Composition

Core: Unalloyed $U$ enriched to $2.1 \% 235 \mathrm{U}$.

Cladding: Nickel-free Zircaloy-2 (25 mils)

End seals: Integral-type, zirconium

C. Dimensions

Over Uniform core, OD

Over end seals, OD

Overall length

Core, tip-to-tip

$\begin{array}{ll}\text { Tube } 148 & \text { Tube } 149 \\ \text { (Inches) } & \text { (Inches) }\end{array}$

D. Extmusion Conditions

NM extrusion number

Tool temperature $\left({ }^{\circ} \mathrm{C}\right)$

2.057

1.699

2.060

Billet temperature $\left({ }^{\circ} \mathrm{C}\right)$

$-$

$118 \quad 118$

$109.8 \quad 108.8$

Extrusion force, running (tons)

" ", maximum (tons)

Tube 148 Tube 149

Extrusion constant, running (tsi)

$\begin{array}{cc}36308 & 36309 \\ 370 & 370 \\ 630 & 630 \\ 738 & 730 \\ 895 & 870 \\ 15.2 & 15.0 \\ 13-15 & 13-15 \\ 15.0 & 15.0\end{array}$

Extrusion ratio

15.0

Billets copper-clad

Copper cutoff 
E. Heat Treatment

Both tubes were beta treated at SRL in their copper extrusion jackets. Treatment consis ted of immersion in a $740^{\circ} \mathrm{C}$ salt bath for 10 minutes followed by quenching in 011 for 20 minutes.

F. Autoclave Test

The tubes were tested for 4 hours at $345^{\circ} \mathrm{C}$ in water followed by 32 hours at $400^{\circ} \mathrm{C}$ in $1500 \mathrm{ps} 1$ steam. Both tubes exhibited a continuous, lustrous, black surface film.

G. Remarks

None.

29. NM Tubes Nos, 150-155 and 157-158

Ref: (1) Progress Reports NMI-7256, 7257, 7258, 7259, 7260.

To further the development of new fuel compositions, short tubular elements were prepared from long extruded tubes. The short elements provided means for irradiating a wider range of compositions and/or heat treatment in a minimum of reactor positions, as well as expediting postirradiation examination. These short tubes were $1.700^{\prime \prime}$ OD X $0.230^{\prime \prime}$ wall X 11 la" long (nominal) with brazed end seals at both ends. A total of 24 short elements consisting of four variations of heat treatment and composition were sent to SRL as irradiation candidates. Fifteen of these tubes, flve from each of tubes 153, 154 and 158, were irradiated in HWCTR. Data for this group of tubes is presented below.

A. Description

Short HWCTR tubuiar elements, 1.700" OD X $0.230^{\prime \prime}$ waII X $11 \frac{1}{4} "$ long (nominal).

B. Composition

$$
\text { Core: } \begin{array}{ll}
150 \text { and } 151 & - \text { Unalloyed urantum } \\
152-154 & -U-350 \mathrm{ppm} F-900 \mathrm{ppm} \mathrm{AI} \\
155 & -U-350 \mathrm{ppm} \mathrm{Fe}-900 \mathrm{ppm} \mathrm{AI-500} \mathrm{ppm} \mathrm{S1-} \\
157 \text { and } 158-\mathrm{U}-350 \mathrm{ppm} \mathrm{Fe}-900 \mathrm{ppm} \mathrm{Al-300} \mathrm{ppm} \mathrm{S1}
\end{array}
$$

Cladding: Zircaloy-2 (22 mils)

End seals: Brazed type, Z1rcaloy-2 


\section{Dimensions}

The following dimensions are typical of the short tubes which were irradiated in HWCTR.

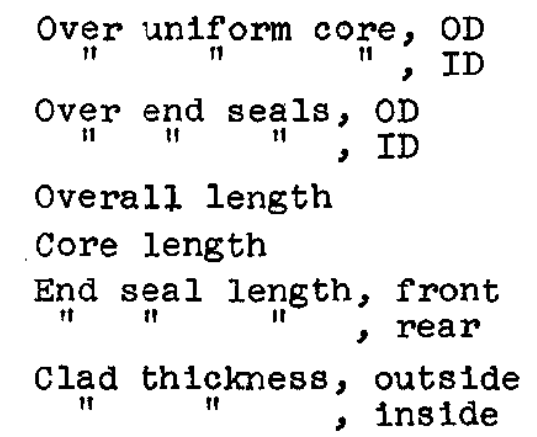

\begin{tabular}{|c|c|c|}
\hline $\begin{array}{l}153-4 \\
\text { (inches) }\end{array}$ & $\begin{array}{l}154-4 \\
\text { (inches) }\end{array}$ & $\begin{array}{l}158-4 \\
\text { (inches) }\end{array}$ \\
\hline $\begin{array}{l}1.690 \\
1.234\end{array}$ & $\begin{array}{l}1.690 \\
1.234\end{array}$ & $\begin{array}{l}1.689 \\
1.234\end{array}$ \\
\hline $\begin{array}{l}1.701 \\
1.231\end{array}$ & $\begin{array}{l}1.700 \\
1.233\end{array}$ & $\begin{array}{l}1.701 \\
1.234\end{array}$ \\
\hline 11.294 & 11.300 & 11.263 \\
\hline 10.721 & 10.750 & 10.713 \\
\hline $\begin{array}{l}0.275 \\
0.275\end{array}$ & $\begin{array}{l}0.275 \\
0.275\end{array}$ & $\begin{array}{l}0.275 \\
0.275\end{array}$ \\
\hline $\begin{array}{l}0.022 \\
0.022\end{array}$ & $\begin{array}{l}0.021 \\
0.022\end{array}$ & $\begin{array}{l}0.022 \\
0.023\end{array}$ \\
\hline
\end{tabular}

\section{Extmusion Conditions}

\begin{tabular}{|c|c|c|c|c|c|c|c|c|}
\hline & \multicolumn{8}{|c|}{ Tube Numbers } \\
\hline & 150 & 151 & 152 & 153 & 154 & 155 & 157 & 158 \\
\hline \multirow{2}{*}{$\begin{array}{l}\text { NM extrusion number } \\
\text { Tool temp. }\left({ }^{\circ} \mathrm{C}\right)\end{array}$} & 36692 & 36693 & 36823 & 36824 & 36825 & 36826 & 37108 & 37109 \\
\hline & ---- & --- & $\ldots$ & -370 & $-\infty-\infty$ & $--\cdots--$ & $-\ldots-$ & $--\cdots$ \\
\hline Billet temp. $\left({ }^{\circ} \mathrm{C}\right)$ & $-\cdots-$ & --- & $-\cdots--$ & -595 & $-\cdots-$ & $-\cdots$ & ---- & $-\cdots-$ \\
\hline $\begin{array}{l}\text { Extrusion force, } \\
\text { running (tons) }\end{array}$ & 750 & 750 & 950 & 910 & 950 & 1130 & 1000 & 960 \\
\hline $\begin{array}{l}\text { Extrusion force, } \\
\text { maximum (tons) }\end{array}$ & 750 & 780 & 1000 & 990 & 980 & 1180 & 1040 & 990 \\
\hline $\begin{array}{l}\text { Extrusion constant, } \\
\text { running (ts1) }\end{array}$ & 15.5 & 15.5 & 19.7 & 19.0 & 19.7 & 23.3 & 20.7 & 19.9 \\
\hline \multirow{2}{*}{$\begin{array}{l}\text { Ram speed (in./min.) } \\
\text { Extrusion ratio }\end{array}$} & 30 & 30 & 42 & 42 & 42 & 42 & 42 & 42 \\
\hline & $---\infty$ & --- & --- & -14.7 & --- & -- & $-1-\infty$ & \\
\hline \multicolumn{9}{|l|}{$\begin{array}{l}\text { B1llets copper-clad } \\
\text { Copper cutoff }\end{array}$} \\
\hline \multicolumn{9}{|c|}{ E. Heat Treatment } \\
\hline \multicolumn{9}{|c|}{$\begin{array}{l}\text { The core stock for the unalloyed U tubes }(150,151) \text { was triple } \\
\text { beta treated followed by a series of extmusions and intermediate } \\
\text { upsets to obtain the desired grain size for extrusion. Prepara- } \\
\text { tion for the cores of tubes } 152 \text {, 153, } 155 \text {, } 157 \text { and } 158 \text { differed } \\
\text { in that they were beta treated-air cooled and beta treated-oil } \\
\text { quenched in place of a triple beta treatment. For tube } 154 \text {, } \\
\text { the core was gamma treated by being held at } 800^{\circ} \text { to } 850^{\circ} \mathrm{C} \text { for } \\
2 \text { hours and furnace cooled. }\end{array}$} \\
\hline
\end{tabular}


Following brazing of the end caps, the completed short tubes made from long tubes 151, 153, 154 and 158 were beta treated at $730^{\circ} \mathrm{C}$ for 15 minutes followed by an oll quench. The ailoyed tubes (from 153, 154 and 158) were then stressrelieved at $500^{\circ} \mathrm{C}$ for 60 minutes followed by air cooling.

F. Autoclave Test

Al1 24 tubes shipped to SRL were tested at $345^{\circ} \mathrm{C}$ in water for 4 hours followed by 32 hours at $400^{\circ} \mathrm{C}$ in 1500 psi steam. The satisfactory quality of the Zircaloy surfaces was indicated by the lustrous, black oxide film that was formed. Exception to this was on the tube ends where the film ranged from dull black to light gray on the weld metal only and was not considered detrimental.

G. Preparation of End Seals

Tube sections of $12 \frac{1}{2} "$ length were cut and faced square, the copper removed by pickling and the ends recessed to a depth of about $0.7^{\prime \prime}$. The recessing was effected by chemical miling over a period of 12 to 14 hours. Next, the tube end was cleaned, etched and a $\frac{1}{2} "$ long Zircaloy-2 end plug inserted with a ring of braze alloy (Zircaloy-2-5 w/ Be) placed on top of 1t. The assembled end closure was placed in a vacuum Induction furnace. Induction heating to $60^{\circ} \mathrm{C}$ above the braze alloy melting point $\left(940^{\circ} \mathrm{C}\right)$ was done in a vacuum of less than 0.5 micron. After a hold of $1 \frac{1}{2}$ minutes at temperature, the furnace was backfilled with dry argon and held at temperature for an additional 3 minutes and cooled. The brazed end was machined to the finished length of $0.275^{\prime \prime}$. Following heat treatment of the tube, the ends were cleaned and TIG-welded in an argon atmosphere.

H. Remarks

None.

I. Current Condition

The s.1x short tubes from 151 and tubes 153-5, 154-3, and 158-2 are stored at SRL w1th only the 151 and 158-2 tubes being sultable for irradiation. Short lengths of tube stock from 151, 153, 154, 155, 157 and 158 are stored at NM. 
30. NM Tubes Nos. $156,159,163-164$

(1) Progress Reports NMI-7258, 7259

(2) NMI-7262, 9/4/64, S.Isserow and others, "Development of

Fabrication Processes for Concentric Driver Tubes for HWCTR".

These four tubes were the prototype outers of a concentric twotube design for the HWCTR drivers to permit operation at higher power levels. Two of the $\mathrm{Zr}-6.5 \mathrm{w} / \mathrm{o}$ natural $\mathrm{U}$ tubes $(159,163)$ were destructively evaluated with tube 164 being used for flow tests at SRI. This two-tube driver tube design was superseded by a single tube driver design (Item 32) for economic reasons. Data for tubes 159 and 163 are presented below.

A. Description

Outer HWCTR driver tube, 2.700" OD X $0.135^{\prime \prime}$ wall (nominal).

B. Composition

Core: Zircaloy-2, $6.5 \mathrm{w} / \mathrm{O}$ U (natural)

Cladding: Zircaloy-2 (15 mils)

End seals: Integral-type, Zircaloy-2

C. Dimensions

(As-extmuded condition).

Outside diameter, average

Inside diameter, average

Outside cladding, average

Tube 159 Tube 163

(inches) (inches)

Core, average

$2.700 \quad 2.703$

$2.424 \quad 2.427$

$0.016 \quad 0.016$

Inside cladding, average

$0.106 \quad 0.105$

$0.016 \quad 0.017$

Overall length, nominal

$119.0 \quad 119.0$

Maximum core, tip-to-tip, nominal $113.0 \quad 113.0$

D. Extrusion Conditions

Tool temperature $\left({ }^{\circ} \mathrm{C}\right)$

B1llet temperature $\left({ }^{\circ} \mathrm{C}\right)$

Extrusion force, muning (tons)

Extrusion force, maximum (tons)

Extrusion constant, running (tsi)

Ram speed (Inches/min)

Extrusion ratio

\begin{tabular}{ccc} 
Tube 159 & & Tube 163 \\
\cline { 1 - 1 } $370-430(1)$ & & $370-430$ \\
755 & & 750 \\
$1050-1150$ & $1050-1150$ \\
1310 & & 1270 \\
$19.1-20.9$ & $19.1-20.9$ \\
33 & 36 \\
16.2 & & 16.2
\end{tabular}

B1llets copper-clad

Copper cutoff

(1) Liner at $430^{\circ} \mathrm{C}$; die, mandrel and cutoff at $370^{\circ} \mathrm{C}$. 
E. Heat Treatment

None.

F. Autoclave Test

None.

G. Remarks

None

H. Current Condition

Tube 164 is stored at SRL.

31. NM Tubes Nos. $160-162,165-166$

Ref: (1) Progress Reports NMI-7258, 7259.

(2) NMI-7262, $9 / 4 / 64$, S.Isserow and others, "Development of Fabrication Processes for Concentric Tubes for HWCTR".

Five inner tubes with four shallow ribs were fabricated as prototypes for the concentric two-tube design for HWCTR drivers. The first three (160-162) used rib stock inserted in the billet as Zircaloy strips. Since only the first of these was satisfactory, two additional inner tubes $(165,166)$ were fabricated with the rib stock machined as part of the outer zircaloy sleeve of the billet. Both of these latter tubes were satisfactory. Tube 160 was used by Dresser Products, Inc., Great Barrington, Mass., to demonstrate that $0.060^{\prime \prime}$ thick full-height $\left(0.300^{\prime \prime}\right)$ ribs could be satisfactorliy electron-beam welded along the full 117.5" length of shallowribbed inner tubes. Work on the concentric two-tube design was superseded by a single tube driver design (Item 32) for economic reasons. Data for tubes 160 and 166 are presented below.

A. Description

Inner HWCTR driver tube, 1.720" OD between ribs, $1.800^{\prime \prime}$ OD across shallow ribs, $0.160^{\circ}$ wa11, and $2.390^{\prime \prime}$ OD rib c1rcle with full-height ribs (nominal).

B. Composition

Core: Z1rcaloy-2, $6.5 \mathrm{w} / \mathrm{O} \mathrm{U}$ (natural)

Cladding: Zircaloy-2 (15 mils)

End seals: Integral-type, Zircaloy-2. 
C. Dimensions

(As-extruded condition)

OD across shallow ribs, average

OD between ribs, average

ID between ribs, average Outside cladaing, average Core, average

Inside cladding, average

overall length, nominal

Maximum core, tip-to-t1p, nominal

Welded rib width

OD across welded ribs, average

Tube 160 Tube 166 (I)

(inches) (inches)

(1) Tube 166 not dimensionaliy evaluated.

(2) Rib stock used for test work was $0.300^{\prime \prime}$ wide $\left(0.285^{\prime \prime}\right.$ width would be used for tubes intended for flow tests or 1rradiation candidates.

D. Extrusion Conditions

Tool temperature $\left({ }^{\circ} \mathrm{C}\right)$

Billet temperature $\left({ }^{\circ} \mathrm{C}\right)$

Extrusion force, running (tons)

$1.811 \quad 1.802$

$1.725 \quad 1.720$

1.395

0.017

0.132

0.017

117.5

113.0

0.060

$2.432(2)$

--

$--$

$-$

$--$

117.5

113.0

Extrusion constant, running (tsi)

Ram speed (Inches/min)

Extrusion ratio

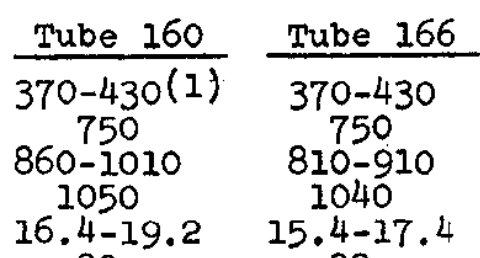

$\begin{array}{cc}30 & 33 \\ 16.9 & 16.9\end{array}$

Billets copper-clad

Copper cutof $f$

(1) Iiner at $430^{\circ} \mathrm{C}$; die, mandrel and cutoff at $370^{\circ} \mathrm{C}$.

E. Heat Treatment

None.

F. Autoclave Test

None

G. Remarks

None

H. Current Condition

Tubes 162,165 and 166 are stored at NM. 
32. NM Tubes Nos, 167-169

Ref: NMI-7263, 11/30/64, J. Slerglef and others, "Development of Fabrication Process for Driver Tubes for the HWCTR".

Three prototype single-tube drivers for HWCTR were fabricated using Zircaloy-2, $11.1 \mathrm{w} / 0$ uranium cores to establish the process for manufacture of a set of 28 tubes for the M-3 fuel set. The design of these tubes is externally identical to the outer tube of the two-tube driver element previously described (Item 30). The uranium in the core alloy was increased from $6.5 \mathrm{w} / 0$ to 11.1 $w / 0$, placing in this tube all the uranium (oralloy, $93 \% 235 \mathrm{U}$, in case of the $M-3$ fuel set) that was originally to be distributed between the two tubes of the earlier design. All three prototypes were destructively evaluated, proving the process to be satisfactory. Data for tubes 167 and 168 are presented below.

A. Description

Single-tube design HWCTR driver tube, 2.700" OD, $0.135^{\prime \prime}$ wa11 (nominal).

B. Composition

Core: Zircaloy-2, $11.1 \mathrm{w} / 0 \mathrm{U}$ (natural).

Cladding: Zircaloy-2 (15 mils).

End seals: Integral-type, Zircaloy-2.

C. Dimensions

(As-pickled condition)

$O D$, average

ID, average

Outside cladding, average

Core, average

Inside cladding, average

overall length, nominal

Core, tip-to-tip

Tube 167 Thube 168

(inches) (inches)

D. Extrusion Conditions

Tool temperature $\left({ }^{\circ} \mathrm{C}\right)$

Billet temperature $\left({ }^{\circ} \mathrm{C}\right)$

Extrusion force, muning (tons)

$2.703 \quad 2.704$

$2.426 \quad 2.428$

$0.015 \quad 0.016$

$0.107 \quad 0.105$

$0.016 \quad 0.017$

$119.0 \quad 119.0$

$110.2 \quad 110.5$

Extrusion constant, running (tsi)

Ram speed (Inches/min)

Extrusion ratio

Tube 167 Tube 168

Billets, copper-clad

Copper cutoff

$\begin{array}{rrr}370 & & 370 \\ 725 & & 720 \\ 1070 & & 1000 \\ 1130 & & 1100 \\ 19.5 & & 18.2 \\ 30 & & 31 \\ 15.4 & & 15.4\end{array}$


E. Heat Treatment

None.

F. Autoclave Test

Half-inch ring sections from the ends of each of the three tubes were tested at $400^{\circ} \mathrm{C}$ in $1500 \mathrm{ps} 1$ steam for 14 days. Rings from tubes 167 and 168 showed indications of copper spots in the cladding as a result of copper-Zircaloy reaction during heating and extrusion of the billets. This was corrected in tube 169 by venting the copper jacket, instead of evacuating and sealing. Confirmation was in the uniform, lustrous, black oxide film on the ring from tube 169 after autoclaving.

G. Remarks

None.

33. NM Tubes Nos. 1-1 to 1-4, 2-1 to 2-3

Ref: DP-943, April 1965, S.R. Nemeth, "Thorium-1.4 w/o 235Uranium Metal Tubes, Fabrication and Irradiation in HWCTR". (Note: DP-943 Supplement contains complete data and had ilmited circulation.)

In connection with studies of $\mathrm{D}_{2} \mathrm{O}$ moderated-and-cooled thorium233uranium breeder reactors, a program was undertaken to deliver to HWCTR two pile-worthy Z1rcaloy-clad tubular elements of a $\mathrm{Th}-1.4 \mathrm{w} / 0$ 235U alloy. Four prototype Th-U tubes $(1-1$ to $1-4)$ and three enriched Th-235U tubes $(2-1$ to $2-3)$ were fabricated. Two of the enriched tubes (2-1 and 2-3) were irradiated in HWCTR, one tube (1-3) used for flow tests at SRL, and two tubes (1-1 and 1-2) destructively evaluated. Data for tubes 1-3, 2-1 and 2-3 are presented below.

\section{A. Description}

Thick-wall thorium-uranium metal fuel tube, 2.545" OD X 0.350 " wall (nominal).

B. Composition

Core: $1-1$ to $1-4$ - Th-1.6 w/o U (natura1)

$2-1$ to $2-3$ - Th-1.5 $\mathrm{w} / \mathrm{O} \mathrm{U}$ enriched to $93 \% 235 \mathrm{U}$.

Cladding: Ziraaloy-2 (30 $\mathrm{m} 1 \mathrm{ls}$ )

End seals: Integral-type, Zircaloy-2 
C. Dimensions

Over uniform core, $O D$

Overall length, nominal

Core tip-to-tip, nominal

D. Extrusion Conditions

(Done at SRP)

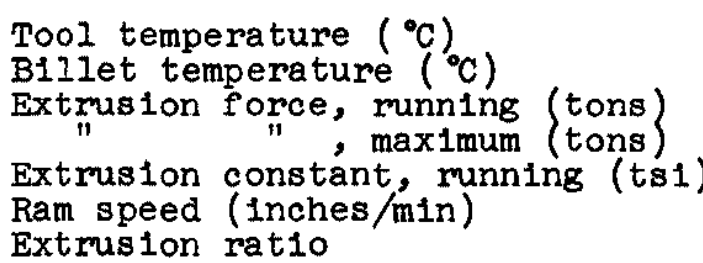

Tube 1-3 Tube 2-1 Tube 2-3 (Inches) (Inches) (inches)

$\begin{array}{lll}2.539 & 2.537 & 2.536 \\ 1.840 & 1.833 & 1.831 \\ 118.0 & 118.0 & 118.0 \\ 108.0 & 108.0 & 108.0\end{array}$

Tube 1-3 Tube 2-1 Tube 2-3

\begin{tabular}{cccc}
\hline $315-370(1)$ & 370 & & 370 \\
775 & 760 & & 760 \\
2000 & 1500 & & 1500 \\
1900 & & 1900 \\
12 & 15 & & 15 \\
14.0 & 12 & & 12 \\
14.0 & & 14.0
\end{tabular}

Billets, copper-clad Copper cutof

(1) Liner and die at $315^{\circ} \mathrm{C}$; mandre $\mathrm{I}$ and cutoff at $370^{\circ} \mathrm{C}$.

E. Heat Treatment

None.

F. Autoclave Test

Tubes 2-1 and 2-3 were tested at $345^{\circ} \mathrm{C}$ in water for 24 hours followed by 24 hours at $400^{\circ} \mathrm{C}$ in 1500 psi steam. Both tubes exhibited a uniform, black oxide surface film.

G. Remarks

None.

H. Current Condition

Tubes $1-3,1-4$ and $2-2$ are stored at SRL; none are satisfactory for 1rradiation. 
34. NM TWOT Tube Series (15 tubes)

Ref: NMI-7218-2, 8/1/65, A. B. Bremer, "Evaluation of Fifteen Outer Tubes of Zircaloy-Clad Unalloyed Uranium for the HWCTR".

Fifteen thin-wall outer tubes with unalloyed uranium cores were fabricated as part of nested concentric tubes for irradiation tests in HWCTR. Elght fuel assemblies containing these tubes were irradiated in HWCTR using spiral ribbon-type spacers. Two of the seven assemblies falled during irradiation because of fretting corrosion where the spacer contacted the tube walls. Data for three typical tubes of this group are presented below.

\section{A. Description}

Thin-wall outer tube, $2.060^{\prime \prime}$ OD X $0.180^{\prime \prime}$ wall (nominal).

B. Composition

Core: Unalloyed uranium

Cladding: Zircaloy-2 (25 mils)

End seals: Integral-type, zirconium

C. Dimensions

Over uniform core, OD

Overall length

Core, tip-to-tip

D. Extrusion Conditions

Tool temperature $\left({ }^{\circ} \mathrm{C}\right)$

Billet temperature $\left({ }^{\circ} \mathrm{C}\right)$

Extrusion force, running (tons)

Extrusion constant, running (tsi)

Ram speed (inches/min)

Extrusion ratio

Billets, copper-clad

Copper cutoff
NM Tube Numbers

\begin{tabular}{ccc}
\multicolumn{3}{c}{ NM Tube Numbers } \\
\begin{tabular}{ccc}
\hline \\
(1nches)
\end{tabular} & $\begin{array}{c}15 \\
\text { (inches) }\end{array}$ & $\frac{1}{\text { (inches) }}$ \\
2.058 & 2.060 & 2.060 \\
1.693 & 1.695 & 1.694 \\
118.0 & 118.0 & 118.0 \\
109.7 & 109.9 & 109.3
\end{tabular}

NM Tube Numbers

\begin{tabular}{|c|c|c|}
\hline 1 & 8 & 15 \\
\hline $\begin{array}{c}370 \\
630 \\
790 \\
983 \\
16.3 \\
13-15 \\
15.0\end{array}$ & $\begin{array}{c}370 \\
630 \\
750 \\
865 \\
15.4 \\
13-15 \\
15.0\end{array}$ & $\begin{array}{c}370 \\
630 \\
773 \\
913 \\
15.9 \\
13-15 \\
15.0\end{array}$ \\
\hline
\end{tabular}


E. Heat Treatment

Each of the fifteen tubes was beta-treated in 1ts copper extrusion jacket while being rotated at $15 \mathrm{rpm}$. Heat treatment consisted of 1mmersion for 10 minutes in $2740^{\circ} \mathrm{C}$ salt bath, air-cooling for 6 minutes, then quenching in $20^{\circ} \mathrm{C}$ water for 2 minutes.

F. Autoclave Test

The tubes were tested for 4 hours in $345^{\circ} \mathrm{C}$ water followed by 32 hours at $400^{\circ} \mathrm{C}$ in $1500 \mathrm{ps} 1$ steam. All tubes had a continuous, lustrous, black surface film following the test.

G. Remarks

None.

H. Current Condition

The tubes of this group that were not irradiated are stored at SRL.

35. NM TWIT Tube Series (15 tubes)

Ref: NM-7218-1, 8/15/62, A.B. Bremer, "Evaluation of Fifteen Inner Tubes of Z1rcaloy-Clad Unalloyed Uranium for the HWCTR".

In conjunction with the TWOT series tubes, fifteen thin-wall inner tubes with unalloyed uranium cores were fabrlcated for irradiation in HWCTR as part of a concentric two-tube design assembly. Two of the seven assemblies irradiated in HWCTR falled because of fretting corrosion where the splral ribbon spacer contacted the fuel tube walls. Data for three typical tubes of this group are presented below.

A. Description

Thin-wall inner tube, 1.020" OD X $0.180 "$ wall (nominal).

B. Composition

Core: Unalloyed uranium

Cladding: Zircaloy-2 (25 mils)

End seals: Integral-type, zirconium 
C. Dimensions

\begin{tabular}{|c|c|c|}
\hline $\begin{array}{c}3 \\
\text { (inches) }\end{array}$ & $\begin{array}{l}\text { Tube Numl } \\
\text { II } \\
\text { (Inches) }\end{array}$ & $\begin{array}{c}18 \\
\text { (1nches) }\end{array}$ \\
\hline $\begin{array}{l}1.022 \\
0.656\end{array}$ & $\begin{array}{l}1.022 \\
0.657\end{array}$ & $\begin{array}{l}1.023 \\
0.658\end{array}$ \\
\hline 118.0 & 118.0 & 118.0 \\
\hline 109.1 & 108.9 & 109.4 \\
\hline
\end{tabular}

D. Extrusion Conditions

Tool temperature $\left({ }^{\circ} \mathrm{C}\right)$

B1llet temperature $\left({ }^{\circ} \mathrm{C}\right)$

Extmusion force, running (tons)

Extrusion constant, running (ts1)

Ram speed (inches/min)

Extrusion ratio

NM Tube Number

$\begin{array}{rrrr}\frac{3}{3} & \frac{11}{11} & \frac{18}{180} & 480 \\ 630 & & 630 & 630 \\ 380 & 373 & 430 \\ 445 & 445 & 550 \\ 13.9 & 13.7 & 15.8 \\ 13-15 & 13-15 & 13-15 \\ 17.7 & 17.7 & 17.7\end{array}$

Billets copper-clad

Copper cutoff

E. Heat Treatment

Each of the fifteen tubes was beta treated in 1ts copper extrusion jacket while being rotated at $15 \mathrm{mpm}$. Heat treatment consisted of immersion for 10 minutes in a $740^{\circ} \mathrm{C}$ salt bath, alr-cooling for 6 minutes, then quenching in $20^{\circ} \mathrm{C}$ water for 2 minutes.

F. Autoclave Test

The tubes were tested for 4 hours in $345^{\circ} \mathrm{C}$ water followed by 32 hours at $400^{\circ} \mathrm{C}$ in $1500 \mathrm{ps} 1$ steam. All tubes had a conti nuous, lustrous, black surface film following the test.

G. Remarks

None.

H. Current Condition

The unirradiated tubes are stored at SRL. 
36. NM Tube Sets $M-1$ and $M-2$, HWCTR Driver Tubes (56 tubes)

Ref: Items 7 and 9 of this appendix.

A total of 56 driver tubes were fabricated for the HWCTR based on the process developed in making the 12 experimental tubes described in Items 7 and 9 . All of these tubes had cores of $\mathrm{Zr}-9.3 \mathrm{w} / 0 \mathrm{U}$ enriched to $93 \% 23 \mathrm{U}$ and Zircaloy-2 end sea is. Twenty-seven tubes (Nos. 1-27) were flad with zircaloy-2, twenty-seven (29-55) with Z1rcaloy-4(1) and two (56-57) were outer-clad with Zircaloy-2 and Inner-clad with Zircaloy-4. The change to Zircaloy-4 was made midway during fabrication in view of the expected lesser amount of hydrogen absorbed by Zircaloy -4 by contrast to Zircaloy -2 .

Three of the 56 tubes were refected as being Irradiation candidates because of minor defects; tube No. 2 , outside surface bump; tube No. 11, depressions in the outer cladding; and tube No. 19, nonbond indication. Of the 13 tubes autoclaved, a11 were found satisfactory.

Forty-eight of the tubes were irradiated in HWCTR, 24 as the $M-1$ driver set and 24 as the $M-2$ driver set. All irradiated tubes performed satisfactorily.

Detalled data for this group of production tubes are similar to that presented under Item 9 of this appendix.

37. NM Tube Set M-3, FWCTR Driver Tubes (28 tubes)

Ref: Item 32 of this appendix.

This production group of 28 single-tube drivers were fabricated for HWCTR based on the process developed in making the three experimental tubes described in Item 32. This set was designed to permit operation of HWCTR at higher power levels than was possible with the M-1 and M-2 driver sets. All of these tubes had cores of Z1rcaloy-2, $11.1 \mathrm{w} / \mathrm{U}$ enriched to $93 \% 235 \mathrm{U}$, Zircaloy-2 cladding and integral-type, Zircaloy-2 end seals.

of the 28 tubes, four were rejected as irradiation candidates for the following reasons. Tube Nos, 20 and 22 had depressions 5 mils deep in the 15-mil-thick cladding. Tube Nos. 23 and 25 falled the notch-fracture test at both ends, with No. 23 also faliling the ultrasonic test for nonbonds. Of the 8 tubes autoclaved, all were found satisfactory.

Detalled data for this group of production tubes are similar to Item 32 .

All 28 tubes are stored at SRL for use in HWCTR, should it be reactivated.

(1) Current designation of this alloy is low nickel-Zircaloy-2 